\title{
Engineered immunogen binding to alum adjuvant enhances humoral immunity
}

\author{
Tyson J. Moyer, ${ }^{1,2}$, Yu Kato ${ }^{2,3}$, Wuhbet Abraham1, Jason Y. H. Chang ${ }^{\circledR 1}$, Daniel W. Kulp ${ }^{2,4,5}$, \\ Nicki Watson ${ }^{6}$, Hannah L. Turner ${ }^{2,5,7}$, Sergey Menis ${ }^{2,5}$, Robert K. Abbott ${ }^{2,3}$, Jinal N. Bhiman ${ }^{2,5,8,9}$, \\ Mariane B. Melo ${ }^{1,2,9}$, Hayley A. Simon ${ }^{3}$, Sara Herrera-De la Mata ${ }^{3}$, Shu Liang3, \\ Gregory Seumois $\mathbb{1}^{3}$, Yash Agarwal ${ }^{1,10}, \mathrm{Na} \mathrm{Li}^{1}$, Dennis R. Burton $\mathbb{1}^{2,5,8,9}$, Andrew B. Ward ${ }^{2,5,7}$, \\ William R. Schief $\mathbb{B}^{2,5,8,9}$, Shane Crotty $\mathbb{1}^{2,3,11}$ and Darrell J. Irvine $\mathbb{B}^{1,2,9,10,12,13 \star}$
}

\begin{abstract}
Adjuvants are central to the efficacy of subunit vaccines. Aluminum hydroxide (alum) is the most commonly used vaccine adjuvant, yet its adjuvanticity is often weak and mechanisms of triggering antibody responses remain poorly understood. We demonstrate that site-specific modification of immunogens with short peptides composed of repeating phosphoserine (pSer) residues enhances binding to alum and prolongs immunogen bioavailability. The pSer-modified immunogens formulated in alum elicited greatly increased germinal center, antibody, neutralizing antibody, memory and long-lived plasma cell responses compared to conventional alum-adsorbed immunogens. Mechanistically, pSer-immunogen:alum complexes form nanoparticles that traffic to lymph nodes and trigger B cell activation through multivalent and oriented antigen display. Direct uptake of antigen-decorated alum particles by B cells upregulated antigen processing and presentation pathways, further enhancing B cell activation. These data provide insights into mechanisms of action of alum and introduce a readily translatable approach to significantly improve humoral immunity to subunit vaccines using a clinical adjuvant.
\end{abstract}

T/ he majority of current vaccines are thought to prevent disease through the induction of a protective antibody response ${ }^{1,2}$. For elicitation of protective humoral immunity by vaccination, B cells must be activated, enter germinal centers to undergo affinity maturation of their antigen receptors and then differentiate into either long-lived plasma cells that secrete antibody constitutively or memory B cells that participate in a recall response on re-exposure to the pathogen. Adjuvants help drive these immunological mechanisms, shaping vaccine protection ${ }^{3,4}$. The most prevalent adjuvant in licensed human vaccines is also one of the oldest known adjuvants, aluminum hydroxide (alum). The function of alum is complex and potentially dependent on induction of inflammatory cell death at the injection site, activation of innate immunesensing pathways and production of chemokines and cytokines at the injection site and/or draining lymph nodes (LNs) ${ }^{5-7}$. Although effective in many vaccines, immune responses elicited by alum are often weaker than other adjuvants in clinical and preclinical studies $^{8-10}$. Despite these limitations, the rigorous requirements for safety in vaccines make the successful development of new adjuvants a major challenge and alum remains an important gold standard benchmark for all adjuvants ${ }^{7}$.

Here we aimed to understand immunological mechanisms of action of this classic adjuvant by evaluating whether the function of alum could be enhanced by engineering the interaction between antigens and alum. Although alum is most commonly employed by adsorbing antigens to alum particles for administration, it is known that many antigens rapidly desorb from alum in the presence of serum or interstitial fluid ${ }^{11-15}$. Additionally, the rate of antigen clearance in vivo is often unaffected by preadsorption to alum ${ }^{16,17}$. We thus designed immunogens for tight binding to alum through site-specific introduction of multivalent phosphoserine (pSer) peptide-polymer affinity tags, which undergo a ligand exchange reaction with the surface of alum to anchor immunogens in an oriented manner on alum particles. We then studied how this modification altered both the fate of these immunogens in vivo and the resulting immune responses.

\section{Results}

Site-specific introduction of pSer affinity tags promotes stable immunogen binding to alum. We designed short peptide and polymer linkers that would mediate binding to alum by ligand exchange between phosphate groups and hydroxyls at the surface of alum particles. Peptides consisting of 1-12 consecutive pSer followed by a short poly(ethylene glycol) spacer and $\mathrm{N}$-terminal maleimide functional group were prepared by solid-phase synthesis (Fig. 1a). We first coupled linkers with one, two or four pSer groups to yeast cytochrome $c$, which has a free solvent-exposed cysteine. The pSermodified cytochrome $c$ exhibited steadily increasing binding to

${ }^{1}$ Koch Institute for Integrative Cancer Research, Massachusetts Institute of Technology, Cambridge, MA, USA. ${ }^{2}$ Consortium for HIV/AIDS Vaccine Development, The Scripps Research Institute, La Jolla, CA, USA. ${ }^{3}$ Division of Vaccine Discovery, La Jolla Institute for Immunology, La Jolla, CA, USA. ${ }^{4}$ Vaccine and Immunotherapy Center, The Wistar Institute, Philadelphia, PA, USA. International AIDS Vaccine Initiative Neutralizing Antibody Center, The Scripps Research Institute, La Jolla, CA, USA. ${ }^{6}$ Whitehead Institute of Biomedical Research, Cambridge, MA, USA. ${ }^{7}$ Department of Integrative Structural and Computational Biology, The Scripps Research Institute, La Jolla, CA, USA. ${ }^{8}$ Department of Immunology and Microbiology, The Scripps Research Institute, La Jolla, CA, USA. ' Ragon Institute of Massachusetts General Hospital, Massachusetts Institute of Technology and Harvard University, Cambridge, MA, USA. ${ }^{10}$ Department of Biological Engineering, Massachusetts Institute of Technology, Cambridge, MA, USA. "Department of Medicine, University of California, San Diego, La Jolla, CA, USA. ${ }^{2}$ Department of Materials Science and Engineering, Massachusetts Institute of Technology, Cambridge, MA, USA. ${ }^{3}$ Howard Hughes Medical Institute, Chevy Chase, MD, USA. *e-mail: djirvine@mit.edu 


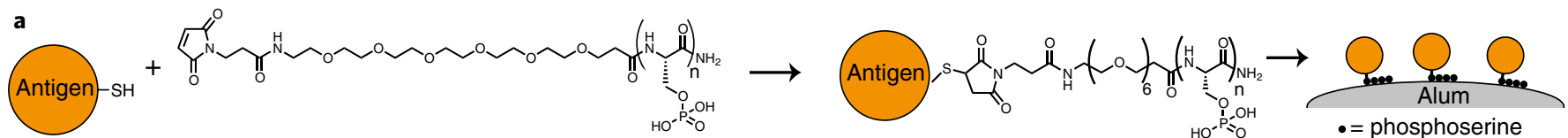

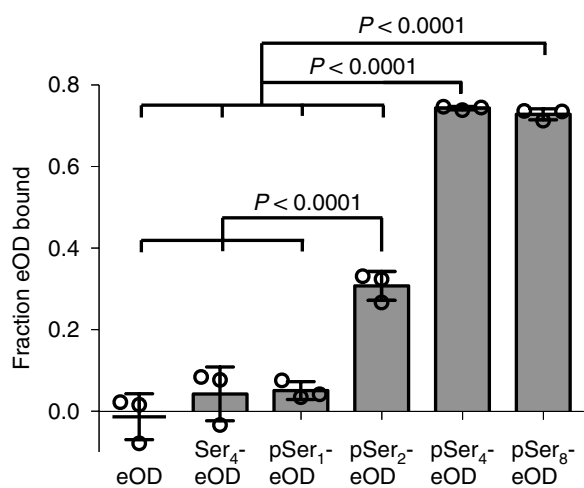

e

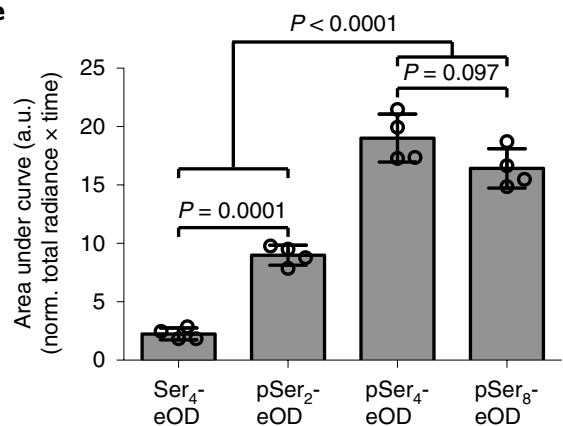
$\mathrm{pSer}_{4^{-}} \mathrm{pSer}_{4^{-}}$ $\mathrm{eOD}+\mathrm{eOD}$ eOD alum + alum + alumP Naive

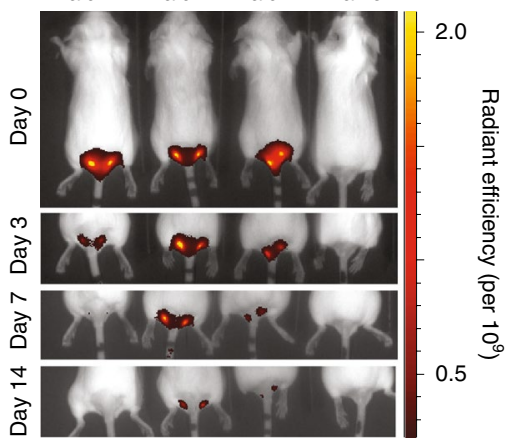

f

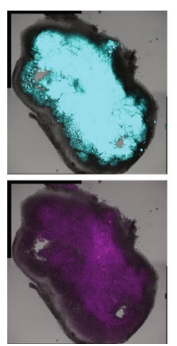

d

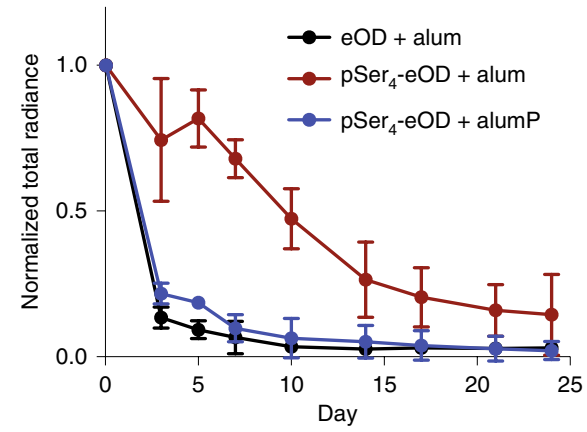

g

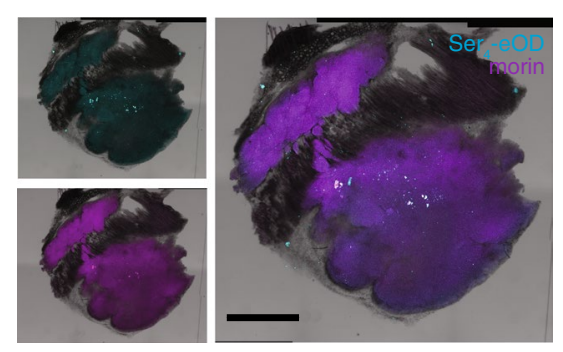

Fig. 1 | Phosphoserine affinity tags enable tunable binding of immunogens to aluminum hydroxide adjuvant. a, Chemical structure of pSer peptidepolyethylene glycol (PEG) affinity tags for site-specific antigen modification. $\mathbf{b}$, Unmodified or pSer-conjugated fluorescent eOD protein (10 $\mu$ g $\mathrm{ml}^{-1}$ ) was mixed with Alhydrogel $\left(100 \mu \mathrm{g} \mathrm{ml}^{-1}\right)$ for $30 \mathrm{~min}$, then incubated in PBS containing $10 \%$ mouse serum for $24 \mathrm{~h}$, followed by fluorescence spectroscopy to measure protein remaining bound to alum. Center values represent mean and error bars represent s.d. ( $n=3$ samples per group). $\mathbf{c}, \mathbf{d}$, Fluorophore-labeled eOD or pSer 4 -eOD (10 $\mu$ g of protein) was mixed with either Alhydrogel (100 $\mu$ g of alum) or aluminum phosphate (alumP) adjuvant (100 $\mu$ ), and injected s.c. in BALB/c mice ( $n=4$ animals per group) followed by longitudinal whole-animal in vivo imaging system (IVIS) imaging of fluorescence at the injection sites. Shown are example whole-animal images (c) and mean total radiance from groups of animals over time (d). Center lines and error bars represent mean and s.d., respectively. e, Fluorophore-labeled eOD proteins (10 $\mu$ g of protein) conjugated with linkers containing $2-8$ pSer residues were mixed with Alhydrogel $(100 \mu \mathrm{g})$ and injected in BALB/c mice followed by IVIS imaging of injection sites over time, as in c ( $n=4$ animals per group). Total fluorescence area under the curve measured over $25 \mathrm{~d}$ was calculated. Center lines represent mean and error bars represent s.d. $\mathbf{f}, \mathbf{g}$, BALB/c mice ( $n=3$ animals per group) injected with eOD:alum or pSer ${ }_{4}-\mathrm{eOD}$ :alum, as in c, were killed $8 \mathrm{~d}$ after injection and immunization sites were analyzed by histology with morin staining to detect alum. Shown are representative injection site cross sections from animals receiving pSer ${ }_{4}$-eOD (f) or eOD ( $\mathbf{g}$ ) with morin in purple, eOD-AF647 in cyan and bright field in gray (scale bars, $1 \mathrm{~mm}$ ). Statistical comparisons in $\mathbf{b}$ and e were made using one-way analysis of variance (ANOVA) followed by Tukey's post hoc test.

alum as the number of serine residues in the tag increased (Extended Data Fig. 1a). To evaluate the role of pSer valency in achieving stable binding to alum, we modified the $240 \mathrm{kDa}$ fluorescent protein phycoerythrin (PE) with 2-20 linkers, where each linker contained either one or four pSer residues (Extended Data Fig. 1b). The pSer ${ }_{1}^{-}$ or $\mathrm{pSer}_{4}$-modified PE was adsorbed to alum, followed by incubation in buffer containing $10 \%$ serum, and protein bound to alum after this two-step process was measured by fluorescence spectroscopy. As shown in Extended Data Fig. 1c, unmodified PE showed almost no retention on alum, but pSer tags promoted a majority of the protein to adhere to alum for $>24 \mathrm{~h}$. Only $2-4 \mathrm{pSer}_{4}$ linkers per protein were required to achieve the same level of binding as 10-20 linkers that had a single pSer residue. Thus, even for very large proteins, modification with a few multivalent pSer tags promotes stable binding to alum in the presence of serum.

To evaluate pSer modification of a bona fide vaccine antigen, we produced a human immunodeficiency virus (HIV) envelope immunogen, eOD-GT8 (eOD hereafter), with site-specific introduction of a free $\mathrm{N}$-terminal cysteine residue for peptide tag coupling (Supplementary Table 1 and Extended Data Fig. 1d). The antigen eOD is a $\sim 20 \mathrm{kDa}$ gp120 engineered outer domain antigen designed to initiate priming of human $\mathrm{B}$ cells capable of evolving toward an important set of $\mathrm{CD} 4^{+}$-binding site-specific broadly neutralizing antibodies (bnAbs) known as VRC01-class antibodies ${ }^{18-21}$. We first prepared eOD antigens coupled with a single peptide linker containing 1-8 phosphoserines or control linkers containing serine residues and evaluated binding to alum. In buffer, $90 \%$ of added eOD adsorbed to alum within $30 \mathrm{~min}$, irrespective of peptide tag composition (Extended Data Fig. 1e). However, when alum-adsorbed eOD was incubated in $10 \%$ serum, only $~ 2 \%$ of unmodified eOD or serine-modified eOD remained bound, whereas pSer-modified antigen showed increasing retention on alum with increasing pSer valency, plateauing at $\sim 75 \%$ retention for four or more phosphoserines (Fig. 1b). Thus, immunogen binding to alum could be readily 

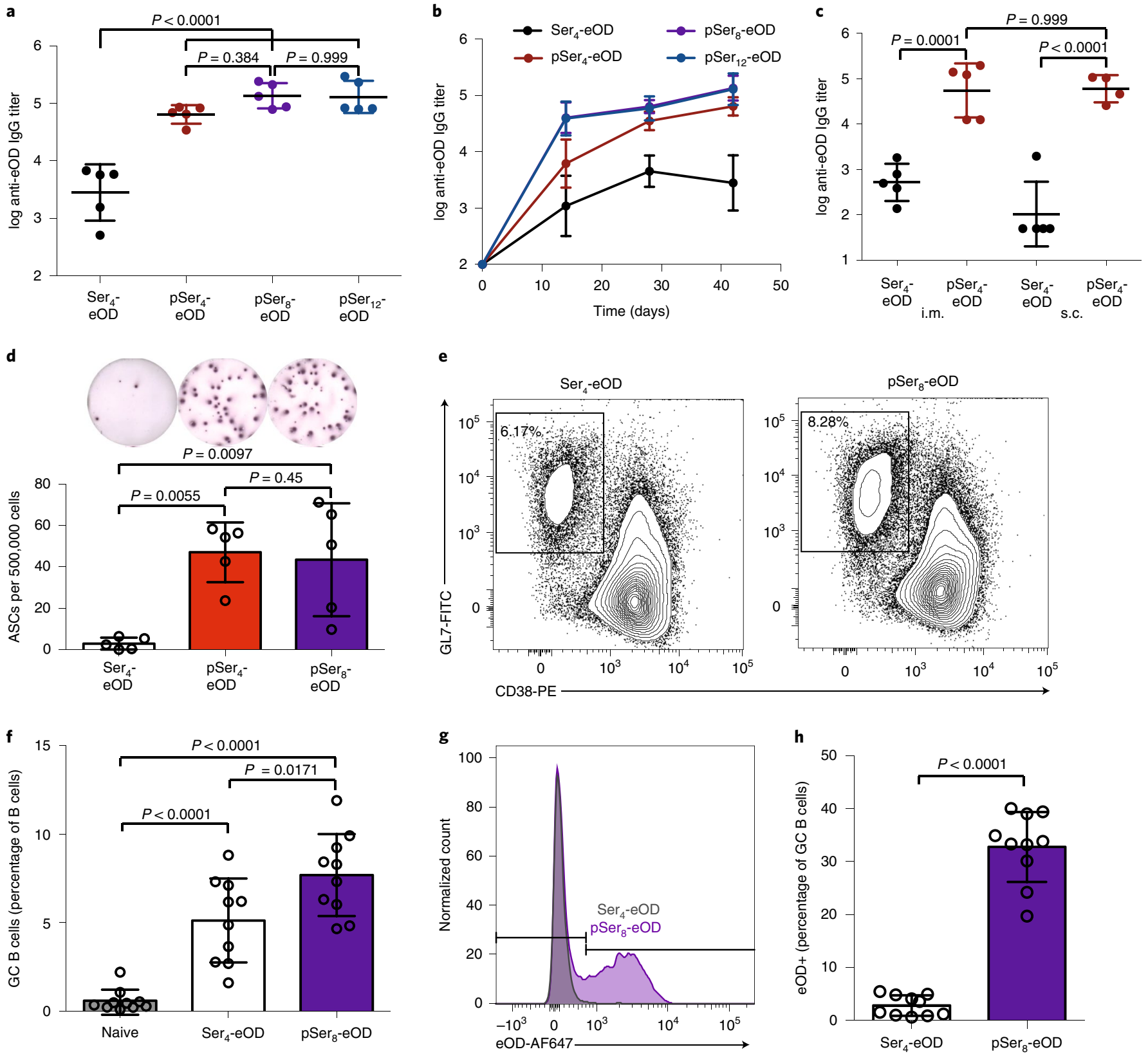

Fig. 2 | Alum-binding pSer antigens elicit enhanced humoral responses in vivo. a,b, BALB/c mice ( $n=5$ per group) were immunized with $50 \mu g$ of alum mixed with $5 \mu \mathrm{g}$ of eOD (with pSer or control Ser tag modification); serum IgG titers were analyzed by ELISA at 6 weeks (a). IgG titers were analyzed over time by ELISA (b). Data are represented as mean \pm s.d. of log-transformed data. c, BALB/c mice ( $n=5$ per group) were immunized with $50 \mu$ g of alum and $5 \mu \mathrm{g}$ of eOD by s.c. or i.m. routes; shown are serum IgG titers at 6 weeks. Center lines represent mean and s.d., respectively of log-transformed data. d, Three months after immunization as in $\mathbf{a}$, eOD-specific antibody-secreting cells (ASCs) from bone marrow were assayed by ELISPOT. Center lines and error bars represent mean and s.d., respectively. $\mathbf{e}-\mathbf{h}, \mathrm{BALB} / \mathrm{c}$ mice $(n=10$ mice pooled from two independent experiments) were immunized with $5 \mu \mathrm{g}$ of eOD and $50 \mu \mathrm{g}$ of alum by s.c. route, and germinal center responses were assayed on day 9 by flow cytometry of dLNs. Shown are representative flow cytometry plots (e) and mean GC B cell frequencies (f), representative histograms ( $\mathbf{g}$ ) and frequencies of GC B cells binding AF647labeled eOD (h). Data in $\mathbf{f}$ and $\mathbf{h}$ are represented as mean \pm s.d. Statistical comparisons in $\mathbf{a}, \mathbf{c}, \mathbf{d}, \mathbf{f}$ were performed using one-way ANOVA followed by Tukey's post hoc test. Comparisons in $\mathbf{a}$ and $\mathbf{c}$ were performed using log-transformed data. Statistical comparison in $\mathbf{h}$ was performed using unpaired, two-tailed Student's t-tests.

tuned through the introduction of pSer affinity tags containing discrete numbers of pSer moieties.

pSer-mediated binding to alum slows immunogen clearance in vivo. Alum is retained at injection sites for many weeks ${ }^{22}$, but clearance of antigens administered with alum is often much faster ${ }^{16,17}$. To determine how pSer-mediated antigen binding to alum influences in vivo antigen availability, $\mathrm{BALB} / \mathrm{c}$ mice were injected with AlexaFluor647 (AF647)-conjugated eOD or $\mathrm{pSer}_{4}-\mathrm{eOD}$ mixed with alum and fluorescence at the injection site was tracked by whole-animal fluorescence imaging. Unmodified eOD cleared from the injection site within $3 \mathrm{~d}$, whereas $\mathrm{pSer}_{4}-\mathrm{eOD}$ persisted for over 3 weeks (Fig. 1c,d). As an additional control, we injected $\mathrm{pSer}_{4}$-eOD mixed with aluminum phosphate, an alternative clinical formulation 
of alum that has many fewer sites for ligand exchange with the pSer tag. In this case, $\mathrm{pSer}_{4}$-eOD cleared at the same rate as eOD (Fig. 1c,d). When varying pSer linker valency, we observed maximum antigen persistence with four or more pSer residues (Fig. 1e). Histology of the injection sites $8 \mathrm{~d}$ after injection showed $\mathrm{pSer}_{8}$ eOD colocalized with alum, whereas unmodified eOD was undetectable (Fig. 1f,g). These results demonstrate that pSer antigens are cleared in vivo substantially more slowly than unmodified antigens adsorbed to alum.

pSer-immunogen:alum immunization enhances multiple facets of the humoral immune response. Control affinity tags with more than four serines were poorly soluble in water, so we chose to compare $\mathrm{pSer}_{\mathrm{n}}-\mathrm{eOD}$ proteins to $\mathrm{Ser}_{4}-\mathrm{eOD}$. Immunization of BALB/c mice with $\mathrm{Ser}_{4}$-eOD:alum or pSer-eOD:alum revealed a clear trend of increasing serum immunoglobulin $(\mathrm{Ig}) \mathrm{G}$ titers as the number of phosphoserines in the affinity tag increased; $\mathrm{pSer}_{8}-\mathrm{eOD}$ elicited 48 -fold higher serum IgG titers compared to the $\mathrm{Ser}_{4}$-eOD antigen, which persisted over 6 weeks (Fig. 2a,b). The pSer-antigen:alum immunization was effective whether vaccines were administered via a subcutaneous (s.c.) or intramuscular (i.m.) route (Fig. 2c) and we did not detect a measurable antibody response to the pSer linker itself (Extended Data Fig. 1f). Further, ELISPOT analysis of bone marrow plasma cells 3 months after a single pSer-antigen:alum immunization showed 16-fold more antigen-specific plasma cells elicited by immunization with $\mathrm{pSer}_{4}-\mathrm{eOD}$ or $\mathrm{pSer}_{8}-\mathrm{eOD}$ compared to control $\mathrm{Ser}_{4}$-eOD (Fig. 2d). Immunization with pSer-modified eOD increased total germinal center (GC) B cells compared to Sermodified eOD by 1.5-fold (Fig. 2e,f and Extended Data Fig. 1g). Strikingly, the percentage of eOD-binding GC B cells increased dramatically from $2.8 \%$ to $33 \%$ in response to pSer-antigen immunization (Fig. 2g,h and Extended Data Fig. 1h). Overall, these results indicate that pSer-immunogen:alum immunization promotes both qualitative and quantitative improvements in humoral immune responses.

Antigen-specific B cells engulf pSer-antigen:alum nanoparticles and exhibit enhanced activation in vitro. Alum is not a monolithic solid but is made up of fibrous aggregates of aluminum hydroxide nanocrystals ${ }^{23}$. In the setting of tight binding between the immunogen and alum particles, we envisioned that antigen delivery to LNs over time could either be mediated by slow release of free antigen from alum surfaces at the site of injection or antigen could be trafficked to LNs still bound to alum nanocrystals (Extended Data Fig. $2 \mathrm{a})$. To assess the potential impact of B cells encountering antigen bound to alum particles, we measured activation of human Ramos $\mathrm{B}$ cells, expressing the eOD-specific germline-inferred VRC01 anti- gen receptor ${ }^{20}$ cultured in vitro with pSer-eOD:alum conjugates versus eOD:alum. Free monomeric eOD elicited a near-baseline calcium signaling response as reported previously ${ }^{20}$, as did $\mathrm{Ser}_{4^{-}}$ eOD mixed with alum (Extended Data Fig. 2b). By contrast, B cells stimulated by pSer-eOD:alum showed increased activation with increasing pSer valency (Extended Data Fig. 2b). B cells incubated with alum and $\mathrm{Ser}_{4}$-eOD bound alum particles but showed little or no eOD uptake, whereas B cells incubated with $\mathrm{pSer}_{8}-\mathrm{eOD}$ :alum internalized alum particles together with eOD (Extended Data Fig. $2 \mathrm{c}, \mathrm{d})$. Higher resolution visualization of these cells by transmission electron microscopy (TEM) imaging revealed that nanoscale alum aggregates were internalized by B cells when $\mathrm{pSer}_{8}-\mathrm{eOD}$ was bound to alum (Extended Data Fig. 3a,b). These results suggest that when bound to alum via pSer linkages, antigens can behave as a multivalent, particulate vaccine that is internalized by B cells.

Alum accumulates in draining LNs and antigen-specific B cells acquire pSer-immunogen bound to alum particles. By separately labeling alum and antigen, we observed that following immunization, $\mathrm{Ser}_{4}$-eOD levels in the LN peaked at $24 \mathrm{~h}$ and rapidly decayed thereafter, whereas alum tracer slowly accumulated (Extended Data Fig. $4 \mathrm{a}-\mathrm{c})$. By contrast, $\mathrm{pSer}_{8}-\mathrm{eOD}$ and alum showed a matching pattern of slow accumulation in draining LNs (dLNs) (Extended Data Fig. 4d). Macrophages took up soluble $\mathrm{Ser}_{4}$-eOD $1 \mathrm{~d}$ after immunization but antigen had cleared from these cells by $7 \mathrm{~d}$. In contrast, macrophages and dendritic cells showed increased uptake of alum and $\mathrm{pSer}_{8}$-eOD after $7 \mathrm{~d}$ (Extended Data Fig. $4 \mathrm{e}-\mathrm{h}$ ). We also directly quantified aluminum levels in dLNs by inductively coupled plasma mass spectrometry. As shown in Extended Data Fig. 4i, aluminum was readily detected in dLNs for both $\mathrm{pSer}_{4}$-eOD:alum and unmodified eOD:alum immunizations.

Given these biophysical findings regarding pSerimmunogen:alum complexes, we hypothesized that alum particles might deliver pSer antigens to B cells in a multivalent form in vivo. To test this possibility, we employed an adoptive transfer model enabling tracking of antigen-specific B cells ${ }^{24}$. Mouse B cells expressing glVRC01 B cell receptors (BCRs) (VRC01 ${ }^{\mathrm{gHL}}$ ) were transferred into wild-type mice ${ }^{24}$, followed by vaccination with alum and $\mathrm{pSer}_{8}$ - or Ser $_{4}$-modified monomeric eOD-GT5, a variant of the eOD immunogen, with a physiological affinity $\left(K_{\mathrm{d}}\right.$ of $\left.\sim 0.3 \mu \mathrm{M}\right)$ for the VRC01 ${ }^{\text {gHL }}$ BCR $^{19,20,24,25}$ (Fig. 3a and Extended Data Fig. 5a). VRC01 ${ }^{\text {gHL }}$ $\mathrm{B}$ cells in the $\mathrm{pSer}_{8}$-eOD-GT5:alum group acquired antigen $1 \mathrm{~d}$ after immunization that continued to accumulate at day 2, whereas $\mathrm{Ser}_{4-}$ eOD-GT5:alum immunization led to nearly undetectable antigen uptake by the eOD-specific B cells (Fig. 3b,c). Histology of spleens showed colocalization of alum and pSer $_{8}-\mathrm{eOD}-\mathrm{GT} 5$ around B cell follicles, whereas in the control group only alum and little to no

Fig. 3 Antigen-specific B cells efficiently take up pSer-antigen bound to alum particles in vivo. a-c, C57BL/ 6 mice adoptively transferred with $1 \times 10^{6}$ $\mathrm{GFP}^{+} \mathrm{CTV}+\mathrm{VRC018 \textrm {HL }} \mathrm{B}$ cells were immunized by intraperitoneal (i.p.) injection of $5 \mu \mathrm{g}$ of AF647-labeled Ser $_{4}$-eOD-GT5 or pSer ${ }_{8}$-eOD-GT5 together with $1 \mathrm{mg}$ of alum. Timeline of adoptive transfer experiment (a). Flow cytometry analysis of splenic VRC018HL B cell binding to AF647-labeled eOD-GT5 in vivo (b). Dotted lines indicate background signal in unimmunized controls. Quantification of AF647-labeled eOD-GT5 fluorescence of splenic VRC018HL B cells

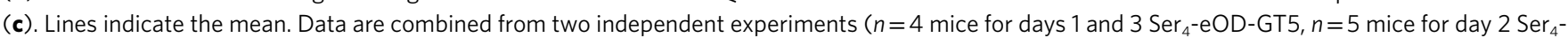
eOD-GT5 and days 1-3 pSer ${ }_{8}$-eOD-GT5). Statistical analysis was performed by a two-tailed Student's $t$-test. d,e, Histological images of spleens from mice $2 \mathrm{~d}$ after immunization by i.p. injection with $10 \mu \mathrm{g}$ of AF647-labeled pSer ${ }_{8}$-eOD-GT5 (d) or Ser ${ }_{4}$-eOD-GT5 (e) and $100 \mu$ g of Cy3-pSer ${ }_{4}$-labeled alum (left scale bar, $1 \mathrm{~mm}$; middle and right scale bars, $100 \mu \mathrm{m} ; n=3$ per group). $\mathbf{f}-\mathbf{h}, \mathrm{C} 57 \mathrm{BL} / 6$ mice adoptively transferred with $1 \times 10^{6} \mathrm{CTV}+\mathrm{VRCO} \mathrm{g}^{\mathrm{HL}} \mathrm{B}$ cells were immunized with $5 \mu$ g of AF647-labeled Ser $_{4}$-eOD-GT5 or pSer ${ }_{8}$-eOD-GT5 and $1 \mathrm{mg} \mathrm{AF488-pSer}{ }_{4}$-labeled alum (alum-AF488) and splenic VRC018HL B cells were analyzed $48 \mathrm{~h}$ after immunization. Representative flow cytometry analysis of splenic VRC018HL B cells (f). Bars represent the mean. Quantification of antigen acquisition by VRC018HL B cells $(\mathbf{g})$. Bars represent the mean. Quantification of alum acquisition by VRC019HL B cells $(\mathbf{h})$. Data are combined from two independent experiments. Statistical comparison was performed using one-way ANOVA with Tukey's post hoc test. i,j, On day 2 following i.p. immunization with $10 \mu \mathrm{g}$ of AF647-labeled pSer ${ }_{8}$-eOD-GT5 with $1 \mathrm{mg}$ of alum, GFP+AF647+VRC01' ${ }^{\text {HL }}$ B cells (i) or GFP-AF647- endogenous B cells (j) were sorted from the spleen, fixed, stained and sectioned for TEM imaging (scale bars, $200 \mathrm{~nm}$ ). Shown are representative images from 145 (i) and 153 (j) cells analyzed. Arrows indicate internalized alum particles. k, Quantification of observed percentage of cell sections positive for alum particles in endogenous (endo) and eOD-GT5+ B cells. Statistical analysis was performed using a two-proportions z-test. 
$\mathrm{Ser}_{4}$-eOD-GT5 was observed (Fig. 3d,e). VRC01 ${ }^{\mathrm{gHL}}$ B cells showed simultaneous uptake of the pSer-eOD-GT5 and alum, contrasting with undetectable levels of either eOD-GT5 or alum uptake in $\mathrm{Ser}_{4^{-}}$ eOD-GT5:alum-immunized mice (Fig. $3 \mathrm{f}-\mathrm{h}$ ). When high-affinity eOD-GT8 was used as the immunogen, pSer modification similarly enhanced antigen (Ag) and alum capture by VRC01 ${ }^{\mathrm{gHL}}$ cells (Extended Data Fig. 5b-f). TEM imaging of flow-sorted antigen ${ }^{+}$
B cells collected from mice immunized with alum:pSer ${ }_{8}$ eOD-GT5 or $\mathrm{pSer}_{8}-\mathrm{eOD}-\mathrm{GT} 8$ showed that VRC01 ${ }^{\mathrm{gHL}} \mathrm{B}$ cells acquiring antigen in vivo had readily detectable accumulations of alum aggregates in endosomal compartments (33\% of 153 cell sections counted), whereas endogenous B cells showed no alum uptake ( 0 of 145 cell sections counted, Fig. 3i-k and Extended Data Fig. 6a-e). Overall, these results demonstrate that by engineering tight binding to alum,

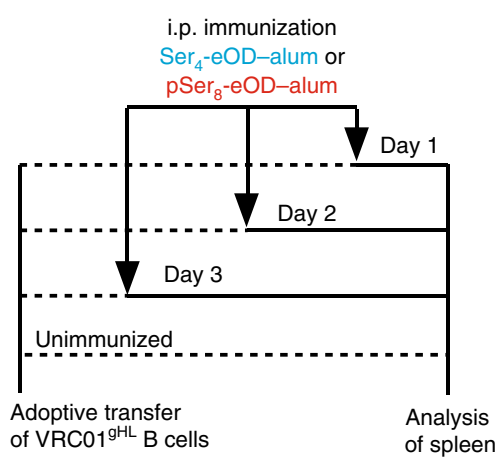

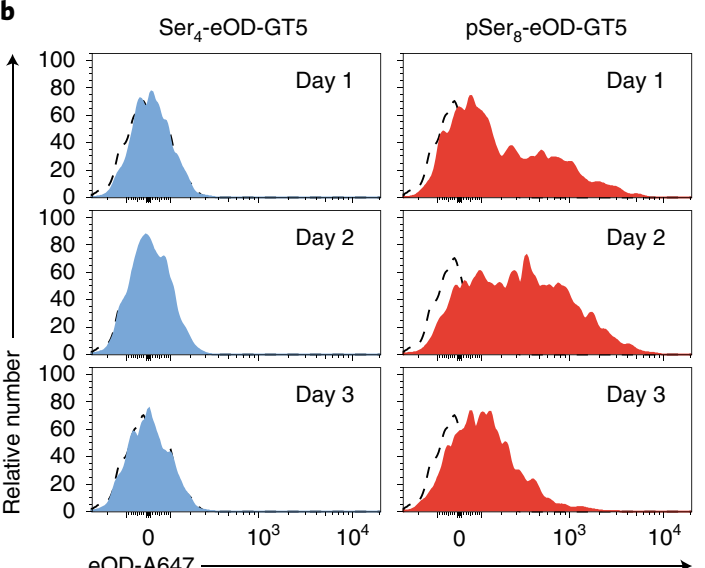
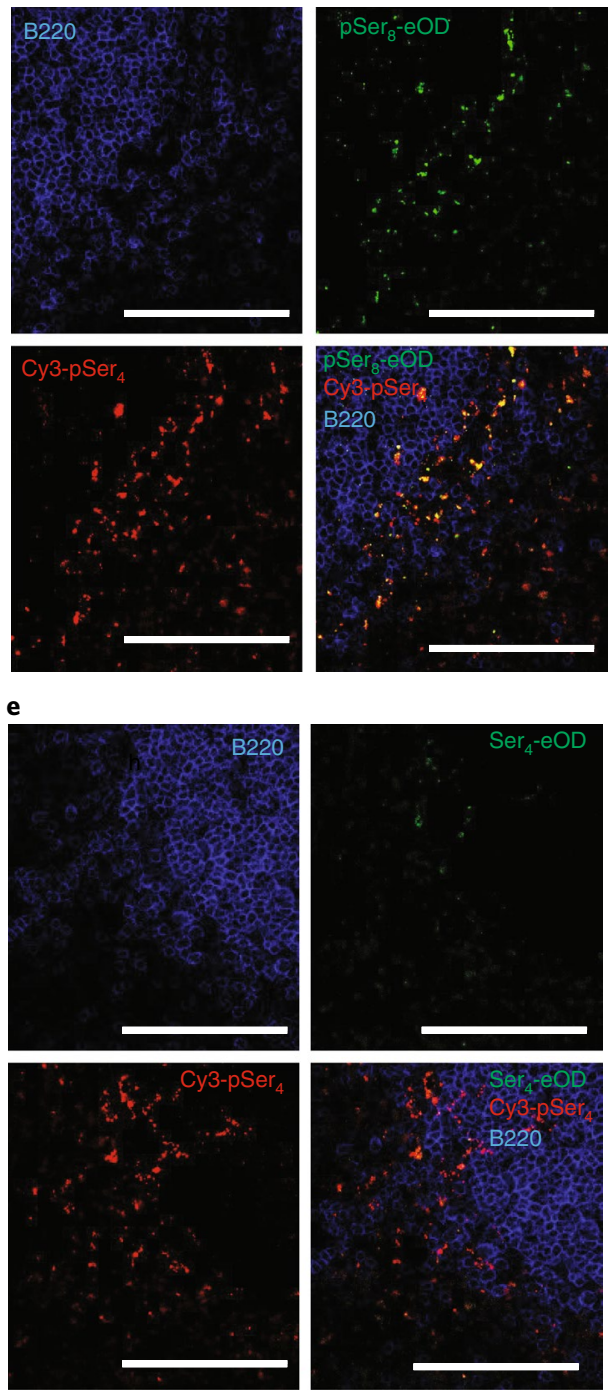

f
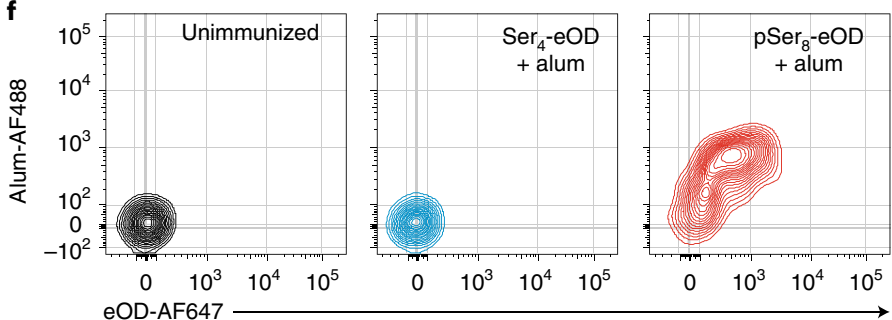

$\mathbf{g}$
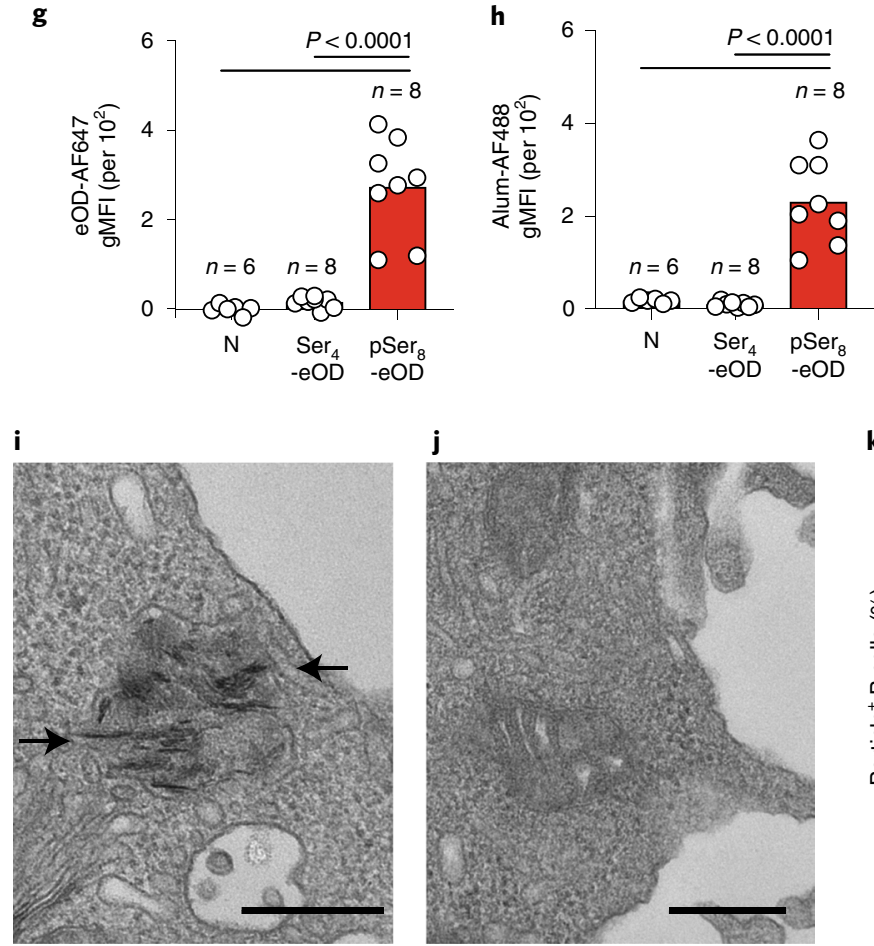

$\mathbf{k}$

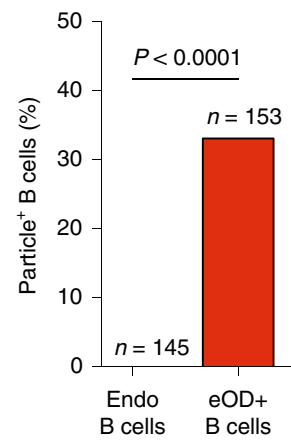


pSer-modified antigens are delivered in alum-bound form to B cells in lymphoid tissue in vivo.

Co-internalization of alum particles and antigen drives activation and antigen-presentation programs in responding $B$ cells. To study the consequences of B cells capturing and internalizing alumbound cognate antigen in vivo, VRC01 ${ }^{\mathrm{gHL}} \mathrm{B}$ cells from mice vaccinated with AF647-labeled pSer $_{8}$-eOD-GT5:alum or AF647-labeled $\mathrm{Ser}_{4}$-eOD:alum were sorted for RNA sequencing (RNA-seq) gene expression profiling (Fig. 4a). AF647 $7^{+}\left(\mathrm{Ag}^{+}\right.$alum $\left.^{+}\right)$VRC01 ${ }^{\mathrm{gHL}} \mathrm{B}$ cells and $\mathrm{AF} 647^{-}\left(\mathrm{Ag}^{-}\right.$alum $\left.{ }^{-}\right) \mathrm{VRC01}{ }^{\mathrm{gHL}} \mathrm{B}$ cells were analyzed independently. At $48 \mathrm{~h}, \mathrm{VRC} 01^{\mathrm{gHL}} \mathrm{B}$ cells from eOD-GT5:alum immunized mice exhibited almost identical gene expression profiles to naive VRC01 ${ }^{\mathrm{gHL}} \mathrm{B}$ cells (Fig. $4 \mathrm{~b}$ and Extended Data Fig. 7a,b). In contrast, over 2,000 genes in $\mathrm{Ag}^{+}$alum ${ }^{+} \mathrm{VRC} 01^{\mathrm{gHL}} \mathrm{B}$ cells were differentially expressed in pSer $_{8}$-eOD-GT5:alum-immunized mice (Fig. $4 \mathrm{~b}$ and Extended Data Fig. 7a,b), indicative of strong activation. Gene signature analysis revealed the $\mathrm{Ag}^{+}$alum ${ }^{+} \mathrm{VRC} 01^{\mathrm{gHL}} \mathrm{B}$ cells were highly metabolically active, with high levels of BCR signaling compared to VRC01 ${ }^{\mathrm{gHL}} \mathrm{B}$ cells from conventionally immunized mice (Fig. 4c-e).

We next assessed whether the stimulatory effect of pSerimmunogen:alum particles on the $\mathrm{B}$ cells in vivo was restricted to BCR signaling or included other pathways. As a control for an immunization that induces activation only through the BCR, we immunized mice with a titrated dose of eOD-GT5-60mer protein nanoparticles alone $\mathrm{e}^{24}$ (Fig. 4a). Interestingly, comparative gene signature analysis revealed selective expression of major histocompatibility complex (MHC) class II antigen-processing and presentation genes in $\mathrm{Ag}^{+}$alum ${ }^{+} \mathrm{B}$ cells from $\mathrm{pSer}_{8}-\mathrm{eOD}-\mathrm{GT} 5$ :alum immunized mice (false discovery rate $q=0.006$, Fig. $4 \mathrm{e}$ ). Single-cell RNA-seq of VRC01 ${ }^{\mathrm{gHL}}$ cells was next utilized for more refined assessment of gene expression. Differentially expressed gene clusters defining VRC01 ${ }^{\mathrm{gHL}} \mathrm{B}$ cells from each immunization condition were identified (Fig. $4 \mathrm{f}$ and Extended Data Fig. 7c). The transcription factor Klf2 was highly differentially expressed in $\mathrm{Ag}^{+}$alum ${ }^{+} \mathrm{B}$ cells and $\mathrm{Klf} 2$ is a known regulator of key genes involved in lymphocyte migration, such as Sell, Ccr7 and S1pr1. Cd83 expression was also highly increased in $\mathrm{Ag}^{+}$alum ${ }^{+} \mathrm{B}$ cells (Fig. 4f,g and Extended Data Fig. 7d). CD83 marks mature antigen-presenting cells and facilitates MHC class II surface expression ${ }^{26}$. CD83 protein was robustly upregulated on $\mathrm{Ag}^{+}$Alum ${ }^{+} \mathrm{VRC01}{ }^{\mathrm{gHL}} \mathrm{B}$ cells from pSer-eOD:alum-immunized mice (Fig. 4h,i and Extended Data Fig. 7e,f), and commensurate increases in MHC class II surface expression were observed (Fig. 4i,j and Extended Data Fig. 7e,f). The major co-stimulatory molecule CD86 was also upregulated (Extended Data Fig. 7g,h). Thus, pSer-immunogen:alum triggers gene expression important for B cell interactions with follicular helper $\mathrm{T}$ cells.

The RNA-seq datasets were then cross compared to stratify genes uniquely regulated by $\mathrm{pSer}_{8}$-eOD-GT5:alum versus eOD-GT560mer and pSer $_{8}-\mathrm{eOD}-\mathrm{GT} 5$ :alum versus $\mathrm{Ser}_{4}$-eOD-GT5:alum (Fig. $4 \mathrm{k})$ to potentially identify alum-induced innate signaling in B cells. Over 300 differentially expressed alum-associated genes were identified, suggesting that alum exhibited direct adjuvant activity in B cells after engulfment. Gene signature analysis did not find evidence of toll-like receptor (TLR) pathways (Fig. 4e). B cells do not express inflammasomes, and deficiencies in interleukin (IL)-18 or NLRP3 did not impair IgG responses to pSer-eOD:alum immunization (Extended Data Fig. 7i,j). Thus, alum nanoparticles seem to directly facilitate activation of B cells through an undefined pathway.

VRC01-class B cells primed by pSer-eOD:alum particles successfully enter germinal centers, undergo somatic hypermutation and generate memory $\mathbf{B}$ cells. We next examined GC responses of VRC01 $1^{\mathrm{gHL}} \mathrm{B}$ cells acquiring $\mathrm{pSer}_{8}-\mathrm{eOD}-\mathrm{GT} 5$ :alum particles (Fig. 5a). VRC01 $1^{\mathrm{gHL}}$ cells primed by $\mathrm{Ser}_{4}-\mathrm{eOD}-\mathrm{GT} 5$ :alum competed poorly in $\mathrm{GCs}^{24}$ (Fig. 5b,c and Extended Data Fig. 8a). In contrast, VRC01 ${ }^{\text {gHL }} \mathrm{B}$ cells constituted $\sim 10 \%$ of total GC B cells after pSer $_{8^{-}}$ eOD-GT5:alum immunization, approximately a 100-fold improvement in competitive fitness over conventional alum immunization (Fig. 5b,c). Additionally, the magnitude of the VRC01 ${ }^{\mathrm{gHL}}$ cell response induced by $\mathrm{pSer}_{8}-\mathrm{eOD}-\mathrm{GT} 5$ :alum approached the level attained using the highly immunogenic eOD-GT5-60mer protein nanoparticle in alum (Fig. 5b,c). Virtually all VRC01 ${ }^{\mathrm{gHL}} \mathrm{GC}$ B cell clones analyzed (295 of 296) from pSer $_{8}$-eOD-GT5:alum ani-

Fig. 4 | B cell activation by alum nanoparticles. a, Schematic representation of the experimental design for GFP+VRCO1gHL $B$ cell isolation (antigen specificity determined using eOD-BV711 probe) for panels b-h and $\mathbf{k}$. VRC01 ${ }^{\mathrm{HH}} \mathrm{B}$ cells were sorted $24 \mathrm{~h}$ after eOD-GT5-60mer immunization, to account for the more rapid drainage of soluble antigen compared to alum-adsorbed antigen. Bulk RNA-seq gene expression analyses are shown in $\mathbf{b}$-e and k. Single-cell RNA-seq gene expression analyses are shown in $\mathbf{f}, \mathbf{g}$. b. Principal-component analysis of RNA-seq of VRC01'HL B cells exposed to different antigen conditions in vivo. Each dot represents an independent replicate. Experimental conditions are represented by colors shown in $\mathbf{a}$ ( $n=4$ mice for eOD-60mer; $n=3$ for all other groups). c, Gene set enrichment analysis (GSEA) of representative cellular metabolic processes in Ag+alum+ ${ }^{+}$RCO1'HL B cells after pSer-eOD:alum immunization in comparison to VRC01' ${ }^{\text {HL }}$ B cells from unprimed mice. The ticks below the line correspond to the rank of each gene. Statistical analysis was performed using a Kolmogorov-Smirnov test. NES, normalized enrichment score. d, GSEA of BCR signaling in $\mathrm{Ag}^{+}$alum ${ }^{+}$VRC01 ${ }^{\mathrm{HL}} \mathrm{B}$ cells after pSer-eOD:alum immunization in comparison to VRC01' ${ }^{\mathrm{HL}} \mathrm{B}$ cells from Ser ${ }_{4}$-eOD:alum immunized mice. Statistical analysis was performed using a Kolmogorov-Smirnov test. e, GSEA for identifying and comparing gene signatures in B cells across all immunization groups compared to B cells from unprimed mice. Red indicates positive association and blue indicates negative association. Circle size is proportional to NES (scale, 1.5-3.0). Color indicates false discovery rate $q$ value (<0.001-0.05). Statistical analysis was performed using Kolmogorov-Smirnov test. UP, upregulated; DN, downregulated. f, Single-cell RNA-seq analysis showing row-wise z scores of differentially expressed genes defining each group. Each column represents a cell. Differentially expressed genes for each group were identified by MAST (mode-based analysis of single-cell transcriptomics) analysis, using 1.25-fold cutoff against the other groups, adjusted $P$ value $<0.05(n=37$ cells for Ser-eOD + alum; $n=37$ cells for pSer-eOD + alum $\left(\mathrm{Ag}^{-}\right) ; n=46$ cells for pSer-eOD + alum $\left(\mathrm{Ag}^{+}\right) ; n=35$ cells for eOD-60mer). Cells were obtained from two mice per group. $\mathbf{g}$, Violin plots showing normalized expression of example genes from single-cell RNA-seq data analyzed in $\mathbf{f}$. Horizontal lines indicate the first quartile, the median and the third quartile. Each circle represents a cell. h, Flow cytometry analysis showing changes in CD62L and CD83 expression patterns on VRC01gHL B cells. Two independent experiments were performed with similar results. i, Bar graphs show geometric mean fluorescence intensity ( $\mathrm{gMFI}$ ) of CD62L, $\mathrm{CD} 83$ and $\mathrm{MHC}$ II on VRC01 ${ }^{\mathrm{gHL}}$ cells in $\mathbf{h}$ and $\mathbf{j}$ normalized to unprimed group. Bars represent the mean $(n=7$ for group 1 and $2, n=6$ for group 3 and $4, n=3$ for group 5). j, Flow cytometry analysis showing changes in MHC II expression patterns on VRC01gHL B cells. Numbers indicate gMFI. Two independent experiments were performed with similar results. k, Scatterplot depicts bulk RNA-seq genes differentially expressed in B cells interacting with alum. The $y$ axis is $\mathrm{Ag}^{+}$alum ${ }^{+} \mathrm{VRC01}{ }^{\mathrm{gHL}} \mathrm{B}$ cells after pSer-eOD:alum immunization versus VRC019HL B cells after Ser-eOD:alum immunization (Ag-alum ${ }^{-} \mathrm{VRCO}^{\mathrm{gHL}} \mathrm{B}$ cells). The $x$ axis is $\mathrm{Ag}^{+}$alum ${ }^{+} \mathrm{VRC0} 1^{\mathrm{gHL}} \mathrm{B}$ cells after pSer-eOD:alum immunization versus VRC01 ${ }^{\mathrm{gHL}} \mathrm{B}$ cells after eOD-60mer immunization in the absence of adjuvant ( $\mathrm{Ag}^{+}$alum ${ }^{-} \mathrm{VRC0}{ }^{\mathrm{gHL}} \mathrm{B}$ cells). Genes significantly (both $x$ axis and $y$ axis, adj $P<0.05$ ) upregulated or downregulated by DESeq2 pairwise comparisons are colored red or blue, respectively. 
mals had acquired at least one heavy chain amino acid mutation $(P<0.0001$, Fig. 5 d), with a substantial fraction $(16.5 \%)$ accumulating five or more amino acid mutations $(P<0.0001$ compared to unvaccinated). Notably, $83 \%$ of VRC $01^{\mathrm{gHL}} \mathrm{HCs}$ acquired the bnAbtype $\mathrm{H} 35 \mathrm{~N}$ substitution $(P<0.0001$ compared to unvaccinated, Fig. 5e), indicating affinity maturation.

To directly test for the development of memory, we measured VRC01 ${ }^{\mathrm{gHL}} \mathrm{GC}$-derived memory $\mathrm{B}$ cells at $\geq \mathrm{d} 30$. VRC01 ${ }^{\mathrm{gHL}}$ GC-derived memory B cells were detected only after pSer $_{8}$ eOD:alum immunization and not after conventional $\mathrm{Ser}_{4}-\mathrm{eOD}$ :alum immunization (Fig. 5f,g and Extended Data Fig. 8b). The majority of $\mathrm{VRC} 01^{\mathrm{gHL}}$ memory B cell clones $(75 \%)$ contained at least one heavy chain amino acid mutation $(P<0.0001$, Fig. $5 d)$ and $58 \%$ contained the bnAb-type $\mathrm{H} 35 \mathrm{~N}$ substitution ( $P=0.003$, Fig. $5 \mathrm{e})$. Overall, pSer-immunogen:alum immunization induced VRC01 ${ }^{\mathrm{gHL}}$ B cell to successfully compete within GCs, undergo affinity maturation and develop into GC-derived memory B cells possessing somatic hypermutation.

Site-specific immobilization of HIV trimer immunogens on alum enables epitope masking. If pSer antigens are accessed by B cells while still bound to alum particles, this opens up an additional strategy for shaping the B cell response, whereby the antigen is immobilized with undesired sites oriented against the alum particle

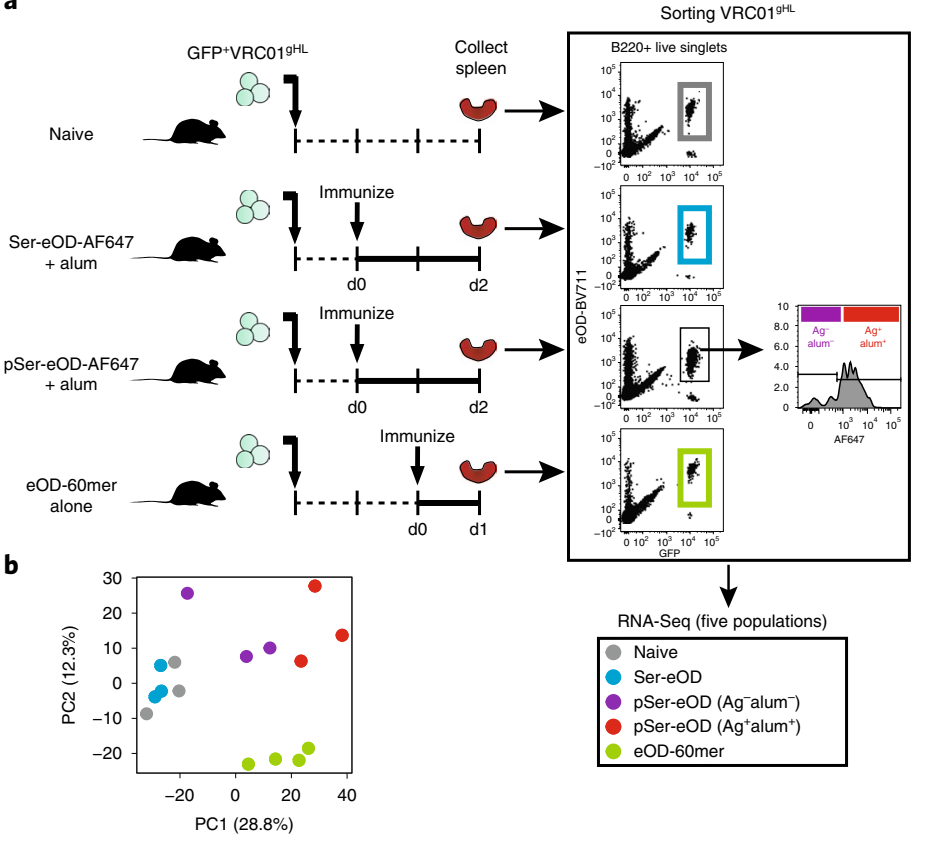

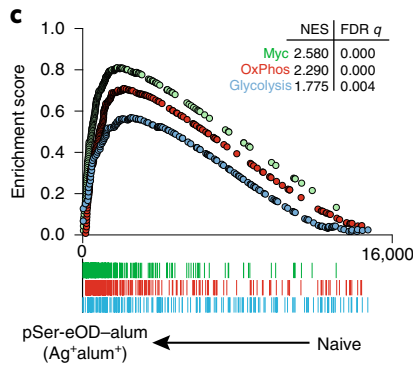

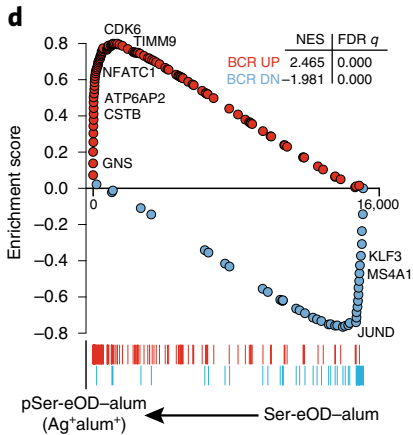

e

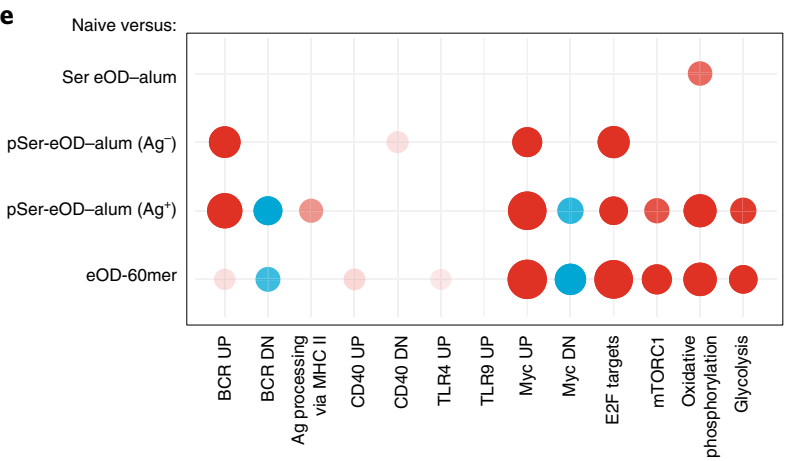

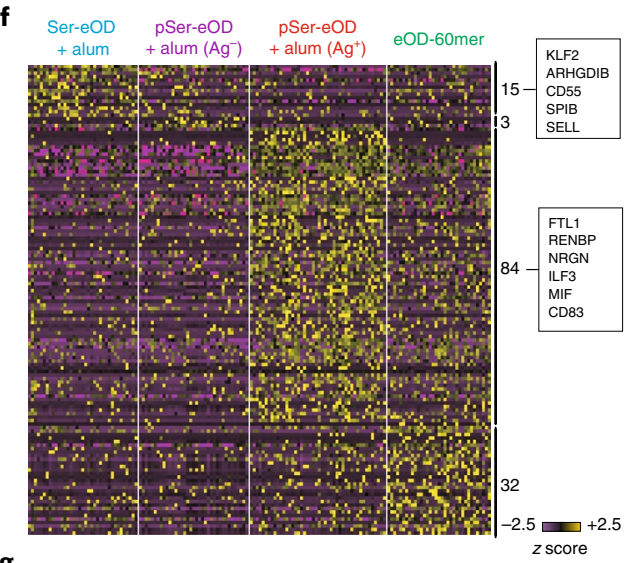

g
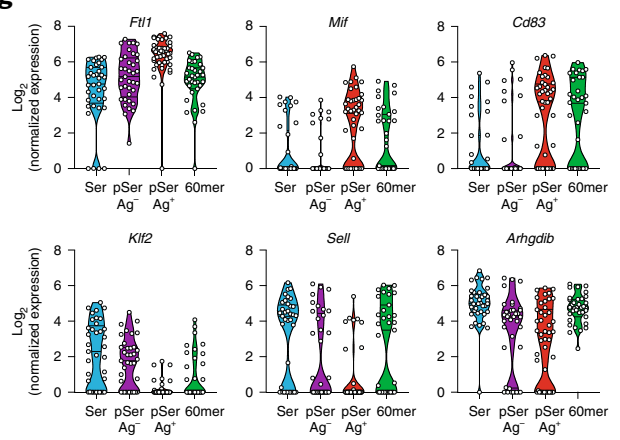
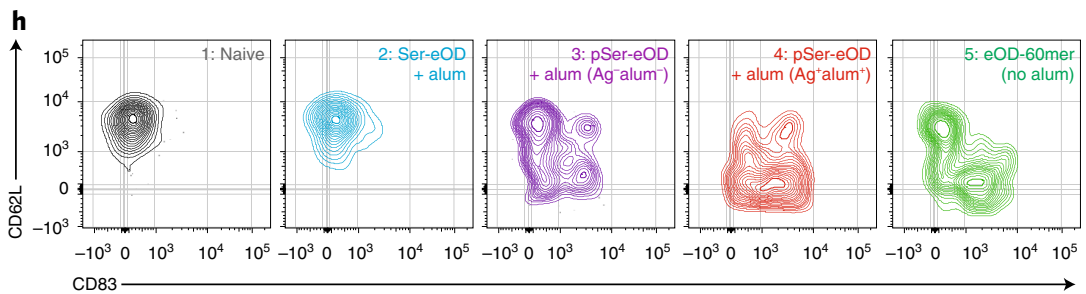

i
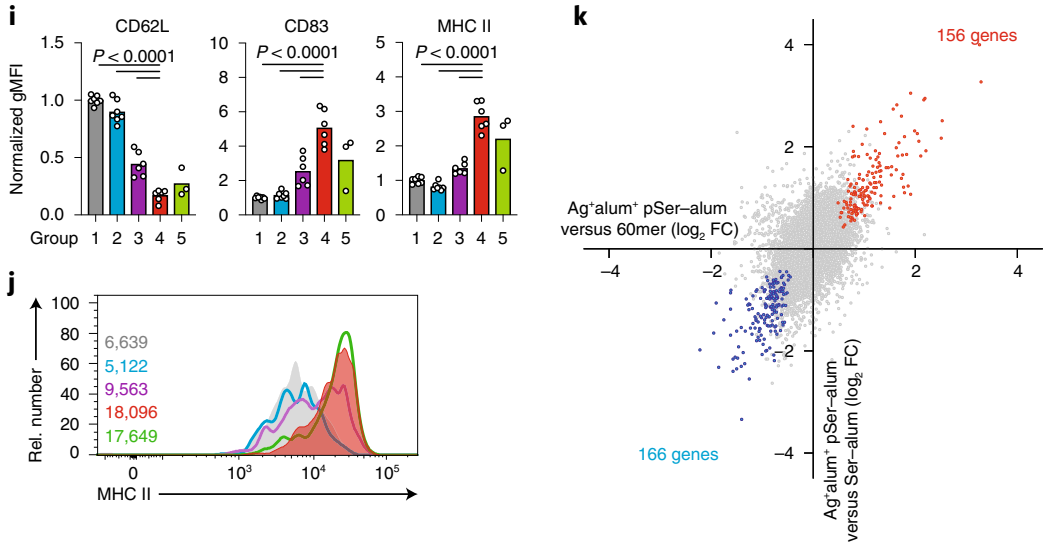


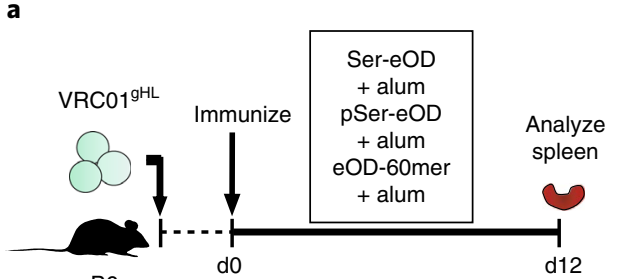

B6

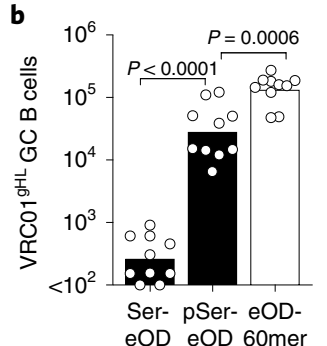

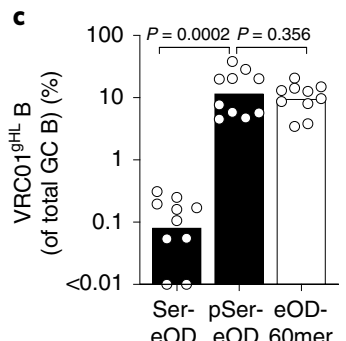

$\mathbf{f}$

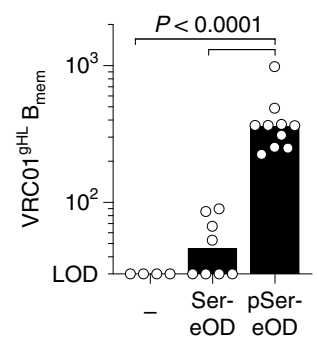

g

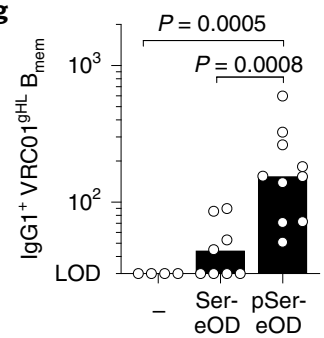

Fig. 5 | pSer-antigen:alum nanoparticles recruit rare B cells to GCs and induce somatic hypermutation and memory. a, Schematic representation of the experimental design for the germinal center experiments in b,c. Data are combined from two independent experiments. Bars indicate the geometric mean ( $n=10$ mice per group). Statistical analysis was performed by one-way ANOVA followed by Tukey's post hoc test on a log-transformed dataset. b, VRC01 ${ }^{\mathrm{HL}} \mathrm{GC}$ B cells per spleen (see Extended Data Fig. 8a for gating). c, Percentage of VRC019HL B cells of total GC B cells in the spleen. $\mathbf{d}-\mathbf{g}$, CD45.1+ B6 mice adoptively transferred with $1 \times 10^{3}$ VRC01 $^{\text {HHL }}$ were left unprimed or immunized with either Ser $_{4}$-eOD-GT5 ${ }_{\text {gp6 }}$ :alum or pSer -eOD-GT5 $_{\text {gp61 }}$ :alum and the spleens were analyzed for VRC01 ${ }^{\mathrm{HHL}}$ memory B cells on d30 or d35. Data are combined from two independent experiments. d,e, Heavy chain mutation analysis of single-cell sorted naive VRC019HL cells, VRC01 GC B cells from d18 pSer-eOD-GT5 ${ }_{\text {gp6 }}$ :alum immunized mice ( $n=296$ cells, four mice), and VRC01 ${ }^{\mathrm{gHL}}$ memory B cells from d35 pSer-eOD-GT5 ${ }_{\text {gp6i }}$ :alum immunized mice ( $n=106$ cells, four mice). d, Circle charts represent the fraction of gVRC01 heavy chain sequences that acquired the indicated number of amino acid mutations. Total number of individual $B$ cell sequences is shown at the center of the circle. e, Circle charts of heavy chain amino acid substitutions at H35 (Kabat numbering). $\mathbf{f}$, GC-derived VRCO1gHL memory B cells ( $B_{\text {mem }}$ ) per spleen. Limit of detection (LOD), 30. Bars indicate the geometric mean (see Extended Data Fig. $8 \mathrm{~b}$ for gating). $\mathbf{g}$, IgG1+ GC-derived VRC018HL memory

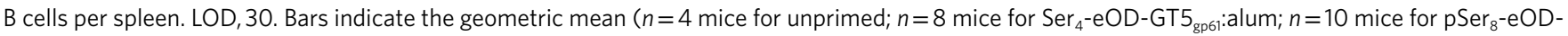
GT5 $_{\text {gp } 61}$ :alum). Statistical analysis was performed by one-way ANOVA followed by Tukey's post hoc test on a log-transformed dataset.

surface. To test this concept, we modified a stabilized gp140 HIV Env trimer, termed MD39 (refs. ${ }^{27,28}$ ), with pSer linkers at the C terminus of each protomer in an effort to minimize antibody responses against the Env trimer base, as these immunodominant responses in animal models are irrelevant for neutralization of the virus ${ }^{29}$ (Fig. 6a and Supplementary Table 2). The pSer $_{4}$-MD39 trimers showed increased retention on alum following serum exposure compared to unmodified MD39 (Extended Data Fig. 9a). IVIS imaging of fluorescent MD39:alum revealed rapid clearance of unmodified MD39 but slow decay of $\mathrm{pSer}_{4}$-MD39 from immunization sites (Extended Data Fig. 9b,c).

A prime and single boost of mice with MD39 mixed with alum elicited a weak trimer-specific IgG response, but $\mathrm{pSer}_{4}{ }^{-}$ MD39 administered in the same regimen led to $\sim 50$-fold higher IgG titers post-boost (Fig. 6b,c). To test whether responses with alum could further be amplified by adding accessory adjuvant compounds, we co-immunized with alum and an ISCOMs-type saponin adjuvant ${ }^{30,31}$ (Extended Data Fig. 9d). Co-administration of alum together with saponin adjuvant allowed MD39 to prime measurable IgG responses following a single immunization, but alum and saponin vaccination with
pSer $_{4}$-MD39 elicited approximately tenfold higher trimer-specific titers that were maintained over time (Fig. $6 \mathrm{~d}$ and Extended Data Fig. 9e). A substantially larger pool of MD39 trimer-specific memory B cells and bone marrow plasma cells were induced by $\mathrm{pSer}_{4}$-MD39:alum compared to control trimer:alum immunization (Extended Data Figs. 9f-i). To compare the alum:antigen-pSer platform to state-of-the-art protein nanoparticles, we immunized $\mathrm{BALB} / \mathrm{c}$ mice with nanoparticle forms of MD39 or eOD with alum. The pSer-antigen immunizations elicited equivalent or slightly higher IgG titers when compared to the protein nanoparticles (Extended Data Fig. 9j,k).

We next evaluated the impact of alum binding on epitope accessibility and the specificity of humoral responses to the Env trimer in vivo. MD39 and $\mathrm{pSer}_{4}$-MD39 were recognized equivalently by both bnAbs (PGT121, PGT151 and 35O22) and non-neutralizing base-specific monoclonal antibody $(\mathrm{mAb})(12 \mathrm{~N})$ when the free trimers were captured on ELISA plates (Fig. 6e, groups 1 and 2). However, when base-modified pSer-MD39 was bound to alum coated on ELISA plates, the $12 \mathrm{~N} \mathrm{mAb}$ against the base of the Env trimer showed substantially reduced binding, whereas bnAbs that recognize a variety of neutralizing sites on the Env trimer surface 
still bound (Fig. 6e). These data indicate that immobilization of the Env trimer on alum particles oriented the trimer to limit accessibility of the Env trimer base.

Next we carried out ELISA analysis of sera from MD39:alumimmunized mice, assessing IgG binding to plate-bound MD39 trimer in the presence or absence of base-binding mAbs. Sera from mice immunized with alum:MD39 showed a modest MD39-specific IgG response and the binding was largely blocked by addition of base-specific mAb (12N) (Fig. 6f,g). By contrast, IgG responses in mice immunized with $\mathrm{pSer}_{4}$-MD39:alum were stronger and only partially reduced by addition of the base-specific mAb (Fig. 6f,g). Furthermore, pSer $_{4}$-MD39:alum immunization led to a significant decrease in responses to the His-tags located at the base of the Env trimer (added for protein purification purposes) when animals were immunized with $\mathrm{pSer}_{4}$-MD39 and increased responses to the gp120 portion of the Env trimer (Fig. 6h and Extended Data Fig. 9l). These results, and those in Fig. 5, suggest that directed orientation of immunogens with the pSer linker can alter the B cell specificity of the immune response.

To assess the impact of limiting base accessibility on the induction of neutralizing antibody responses against the trimer, we immunized rabbits with MD39 or pSer $_{8}$-MD39 and alum. The $\mathrm{pSer}_{8}$-MD39:alum immunization resulted in higher binding IgG titers to both the whole trimer and to gp120, when compared to an unmodified MD39:alum control, particularly after prime (Fig. 6i,j). The pSer-trimer:alum immunization also significantly enhanced the induction of autologous tier-2-neutralizing IgG responses, with six of six animals generating tier 2 neutralizing antibody titers at week 10 ( 2 weeks after boost). In contrast only 3 of 6 rabbits in the MD39:alum control group elicited neutralization at this point and mean neutralizing antibody titers for the responders were 6.5-fold lower (Fig. 6k,l and Extended Data Fig. $9 \mathrm{~m})$. To determine whether this enhanced induction of neutralization was linked to alterations in the specificity of the antibody response, we carried out TEM imaging of complexes formed between soluble MD39 Env trimer and purified IgG of the immunized animals at week 10 (ref. ${ }^{32}$ ). This analysis revealed an antibody response dominated by base-binding IgGs in the MD39:alum group, but a more diverse response in the $\mathrm{pSer}_{8}-\mathrm{MD} 39$ :alum group that included putative neutralization epitopes in gp120 and the gp41-gp120 interface (Fig. 6m,n and Extended Data Fig. 10a,b). Thus, oriented antigen immobilization on alum as an inorganic nanoparticle scaffold enables B cell targeting of complex antigens to be shifted toward protective epitopes.

\section{Discussion}

Here we show that alum, the adjuvant with the longest and most widespread clinical usage, can be modified to both deliver antigen in a particulate form and provide sustained antigen delivery to LNs, simply by engineering the binding of immunogens to alum particles through site-specific introduction of phosphate-bearing peptide linkers. This approach was inspired by work of Hem and colleagues, who discovered that phosphorylated proteins were much more tightly bound to alum than antigens relying solely on electrostatic or hydrogen bonding interactions $s^{12,13}$. Following on from these observations, several studies compared humoral responses primed by phosphorylated antigens with different levels of alum-binding affinities and found disparate conclusions, where increasing alumbinding affinity either increased, decreased or had no impact on the magnitude of the IgG response, depending on the antigen and experimental details $s^{11,16,33,34}$. We suspect these conflicting findings may have resulted from the use of antigens randomly modified over the entire protein surface with alum-binding sites, which may allow the immunogen to crosslink alum particles together or become denatured upon binding, either of which could have unpredictable effects on the immune response.

Uptake of antigen-displaying alum particles has multiple implications for the $\mathrm{B}$ cell response beyond enhanced crosslinking of BCRs, as alum activates innate immunity signaling pathways ${ }^{35-38}$. In addition, alum can activate complement ${ }^{39,40}$, triggers activation of antigen-presenting cells (APCs) through interactions with the plasma membrane $e^{41}$ and induces sustained antigen presentation by APCs in vitro ${ }^{42}$. Through RNA sequencing of sorted antigen ${ }^{+}$alum ${ }^{+}$ antigen-specific B cells, we found evidence that acquisition of antigen bound to alum drives strong activation of BCR signaling, antigen processing and presentation and metabolism pathways.

Fig. 6 | Enhanced humoral responses to HIV Env trimer immunogens elicited by pSer-antigen:alum immunization. a, Schematic of pSer conjugation to the base of MD39 trimer immunogens. b,c, BALB/c mice ( $n=5$ per group) were immunized with $2 \mu$ g of MD39 (with or without pSer modification) mixed with $50 \mu \mathrm{g}$ of alum at days 0 and 21. b. Serum IgG titers are shown over time. Statistical comparison by two-way ANOVA followed by a Bonferroni test of the log-transformed data. Data are represented as mean \pm s.d. of the log-transformed data. $\mathbf{c}$, lgG titers from individual mice on day 63 . Center value and error bars represent mean and s.d., respectively of the log-transformed data. d, BALB/c mice ( $n=5$ per group) were immunized with $5 \mu g$ of MD39 or pSer 4 -MD39 mixed with $50 \mu$ g of alum and $5 \mu$ g of saponin adjuvant. Serum IgG titers are shown over time. Statistical comparison by two-way ANOVA followed by a Bonferroni test. Data are represented as mean \pm s.d. of the log-transformed data. e, Antigenicity analysis of MD39 trimer captured

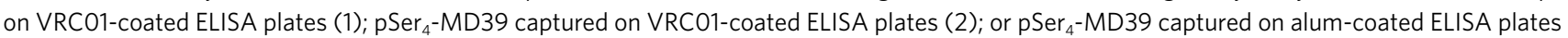
(3). Binding replicates $(n=3)$ are shown from one representative experiment, which was performed in duplicate. Shown are raw ELISA absorbances for binding of indicated monoclonal antibodies added at $0.1 \mu \mathrm{g} \mathrm{m} \mathrm{m}^{-1}$. Center value and error bars represent mean and s.d., respectively. Statistical analysis was performed using one-way ANOVA followed by a Tukey's post hoc test. $\mathbf{f}-\mathbf{h}, \mathrm{BALB} / \mathrm{c}$ mice $\left(n=5\right.$ mice) were immunized with $2 \mu \mathrm{g}$ of MD39 or pSer ${ }_{4}-\mathrm{MD} 9$ mixed with $50 \mu \mathrm{g}$ of alum on days 0 and 21. Raw ELISA dilution curves for day 63 MD39-specific lgG assessed in the presence or absence of $20 \mu g$ ml ${ }^{-1}$ competing base-binding monoclonal Ab $12 \mathrm{~N}$. Data are represented as mean \pm s.e.m. (f). Area under the curve of ELISA signal in the presence of $12 \mathrm{~N}$ Ab (normalized to area under the curve in the absence of base-blocking $A b)(\mathbf{g})$. Center lines and error bars represent mean and s.d., respectively. Two data points were removed from analysis in the MD39 group because of low titers. MD39 gp120-specific lgG titers are shown at day 63 . Center value and error bars represent mean and s.d., respectively of the log-transformed data (h). i-I, New Zealand white rabbits ( $n=6$ per group) were immunized on days 0,56 and 112 by bilateral s.c. injection with $1 \mathrm{mg}$ of alum and $100 \mu \mathrm{g}$ of MD39 or $100 \mu \mathrm{g}$ of pSer - MD39 per animal. Overall MD39-binding IgG titer assessed by ELISA as a function of time (i). Data are represented as mean \pm s.d. Center value and error bars represent mean and s.d., respectively of the log-transformed data. gp120-specific titer measured by ELISA at week 10 (j). Center value and error bars represent mean and s.d., respectively of the logtransformed data. Neutralization titers, assessed as the serum dilution required to neutralize $50 \%$ of the autologous tier 2 virus, measured as a function of time (k). ID $D_{50}$, infective dose. Center lines are represented as mean and s.d., respectively. Neutralization titers at week 8 (after prime) and week 10 ( 2 weeks after boost) (I). Center value and error bars represent mean and s.d., respectively. m,n, Single-particle EM analysis of polyclonal lgG antibodies

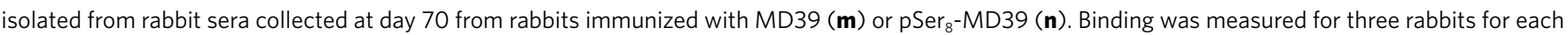
immunization condition and representative images are shown. Statistical tests used were unpaired, two-tailed Student's $t$-test of the log-transformed data $(\mathbf{c}, \mathbf{h}, \mathbf{j})$ and a two-tailed Mann-Whitney U-test $(\mathbf{g}, \mathbf{I})$. 
We also demonstrated that site-specific introduction of alumbinding pSer groups enables the epitope specificity of antibody responses to an antigen to be shifted away from immunodominant but nondesired epitopes. While the generality of this strategy remains to be demonstrated for additional antigens beyond the HIV envelope, this approach may be particularly relevant for vaccines targeting viral a

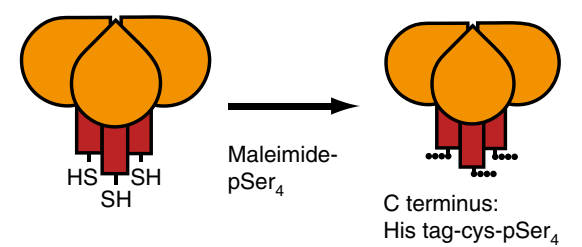

d
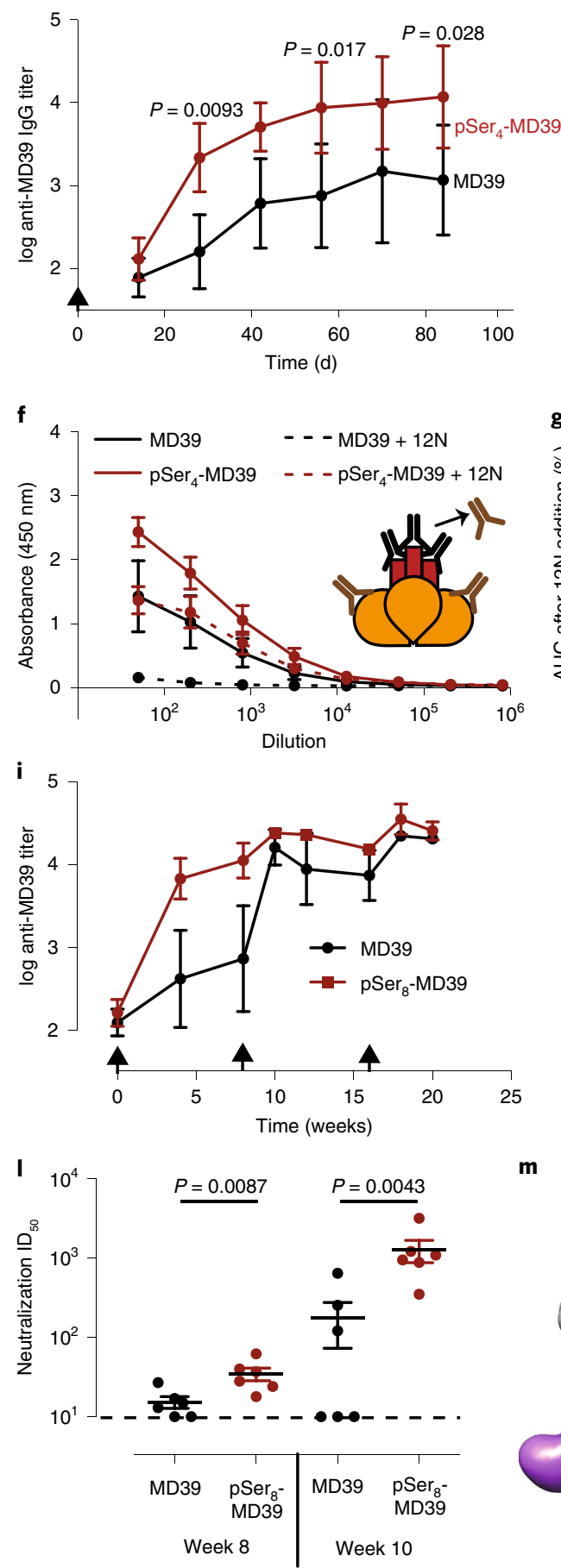
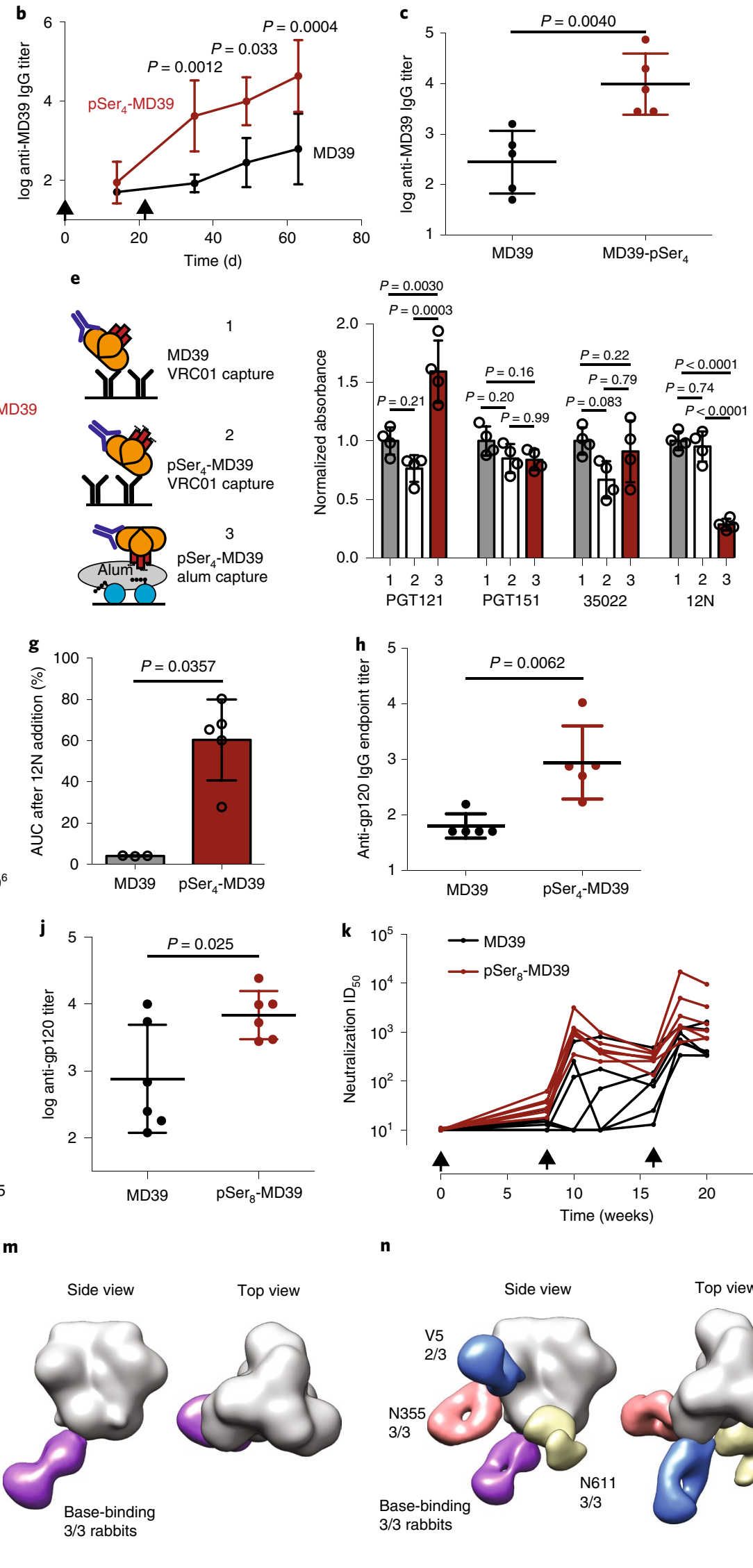
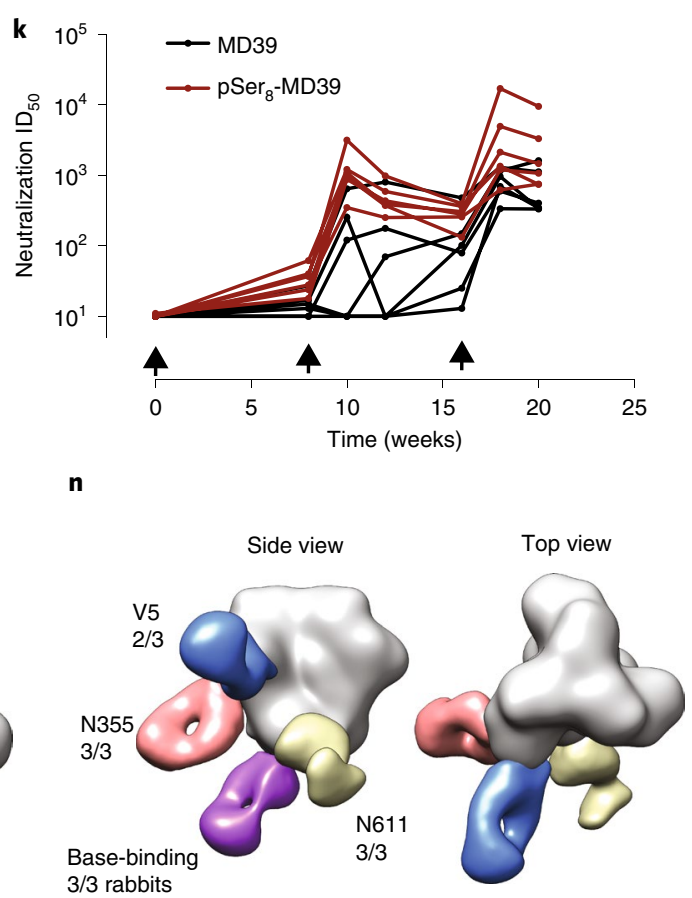
envelope entry receptors. Soluble forms of these proteins typically expose a large surface at the envelope spike base that harbors nonneutralizing epitopes. In the case of HIV Env, the trimer base is an irrelevant target for protective humoral immunity but has been found in animal models to be highly immunogenic ${ }^{43}$, raising concerns that immunodominant base-directed antibody responses might interfere with the induction of desired neutralizing antibody lineages ${ }^{29}$. Oriented antigen display on alum using pSer modification provides one strategy to overcome this issue. Notably, for the HIV gp120 and gp140 trimer immunogens studied here, we routinely achieved yields following peptide coupling and purification of $75-85 \%$, with essentially quantitative substitution of the terminal cysteines. There is likely substantial room for further optimization but these outcomes are promising from a translational immunogen manufacturing perspective and we expect this method will be broadly applicable to diverse subunit vaccines in development for infectious disease and beyond.

\section{Online content}

Any methods, additional references, Nature Research reporting summaries, source data, extended data, supplementary information, acknowledgements, peer review information; details of author contributions and competing interests; and statements of data and code availability are available at https://doi.org/10.1038/s41591020-0753-3.

Received: 6 June 2019; Accepted: 6 January 2020; Published: 17 February 2020

\section{References}

1. Rappuoli, R. \& Aderem, A. A 2020 vision for vaccines against HIV, tuberculosis and malaria. Nature 473, 463-469 (2011).

2. Plotkin, S. A. Correlates of protection induced by vaccination. Clin. Vaccine Immunol. 17, 1055-1065 (2010).

3. Reed, S. G., Orr, M. T. \& Fox, C. B. Key roles of adjuvants in modern vaccines. Nat. Med. 19, 1597-1608 (2013).

4. Moyer, T. J., Zmolek, A. C. \& Irvine, D. J. Beyond antigens and adjuvants: formulating future vaccines. J. Clin. Invest. 126, 799-808 (2016).

5. Kool, M., Fierens, K. \& Lambrecht, B. N. Alum adjuvant: some of the tricks of the oldest adjuvant. J. Med. Microbiol. 61, 927-934 (2012).

6. McKee, A. S. \& Marrack, P. Old and new adjuvants. Curr. Opin. Immunol. 47, 44-51 (2017).

7. HogenEsch, H., O’Hagan, D. T. \& Fox, C. B. Optimizing the utilization of aluminum adjuvants in vaccines: you might just get what you want. NPJ Vaccines 3, 51 (2018).

8. Francica, J. R. et al. Analysis of immunoglobulin transcripts and hypermutation following SHIV(AD8) infection and protein-plus-adjuvant immunization. Nat. Commun. 6, 6565 (2015).

9. Khurana, S. et al. Vaccines with MF59 adjuvant expand the antibody repertoire to target protective sites of pandemic avian $\mathrm{H} 5 \mathrm{~N} 1$ influenza virus. Sci. Transl. Med. 2, 15 ra15 (2010).

10. Leroux-Roels, G. et al. Impact of adjuvants on $\mathrm{CD} 4\left(^{+}\right) \mathrm{T}$ cell and B cell responses to a protein antigen vaccine: results from a phase II, randomized, multicenter trial. Clin. Immunol. 169, 16-27 (2016).

11. Hansen, B., Sokolovska, A., HogenEsch, H. \& Hem, S. L. Relationship between the strength of antigen adsorption to an aluminum-containing adjuvant and the immune response. Vaccine 25, 6618-6624 (2007).

12. Morefield, G. L. et al. Effect of phosphorylation of ovalbumin on adsorption by aluminum-containing adjuvants and elution upon exposure to interstitial fluid. Vaccine 23, 1502-1506 (2005).

13. Iyer, S., HogenEsch, H. \& Hem, S. L. Relationship between the degree of antigen adsorption to aluminum hydroxide adjuvant in interstitial fluid and antibody production. Vaccine 21, 1219-1223 (2003).

14. HogenEsch, H. Mechanisms of stimulation of the immune response by aluminum adjuvants. Vaccine 20 (Suppl. 3), S34-S39 (2002).

15. Weissburg, R. P. et al. Characterization of the Mn Gp120 Hiv-1 vaccine: antigen-binding to alum. Pharmaceut. Res. 12, 1439-1446 (1995).

16. Noe, S. M., Green, M. A., HogenEsch, H. \& Hem, S. L. Mechanism of immunopotentiation by aluminum-containing adjuvants elucidated by the relationship between antigen retention at the inoculation site and the immune response. Vaccine 28, 3588-3594 (2010).

17. Gupta, R. K., Chang, A. C., Griffin, P., Rivera, R. \& Siber, G. R. In vivo distribution of radioactivity in mice after injection of biodegradable polymer microspheres containing 14C-labeled tetanus toxoid. Vaccine 14, 1412-1416 (1996).
18. Sok, D. et al. Priming HIV-1 broadly neutralizing antibody precursors in human Ig loci transgenic mice. Science 353, 1557-1560 (2016).

19. Jardine, J. G. et al. HIV-1 vaccines. Priming a broadly neutralizing antibody response to HIV-1 using a germline-targeting immunogen. Science 349, 156-161 (2015).

20. Jardine, J. et al. Rational HIV immunogen design to target specific germline B cell receptors. Science 340, 711-716 (2013).

21. Jardine, J. G. et al. HIV-1 broadly neutralizing antibody precursor B cells revealed by germline-targeting immunogen. Science 351, 1458-1463 (2016).

22. Flarend, R. E. et al. In vivo absorption of aluminium-containing vaccine adjuvants using 26Al. Vaccine 15, 1314-1318 (1997).

23. Harris, J. R. et al. Alhydrogel(R) adjuvant, ultrasonic dispersion and protein binding: a TEM and analytical study. Micron 43, 192-200 (2012).

24. Abbott, R. K. et al. Precursor frequency and affinity determine B cell competitive fitness in germinal centers, tested with germline-targeting HIV vaccine immunogens. Immunity 48, 133-146 (2018).

25. Havenar-Daughton, C. et al. The human naive B cell repertoire contains distinct subclasses for a germline-targeting HIV-1 vaccine immunogen. Sci. Transl. Med. 10, eaat0381 (2018).

26. Tze, L. E. et al. CD83 increases MHC II and CD86 on dendritic cells by opposing IL-10-driven MARCH1-mediated ubiquitination and degradation. J. Exp. Med. 208, 149-165 (2011).

27. Kulp, D. W. et al. Structure-based design of native-like HIV-1 envelope trimers to silence non-neutralizing epitopes and eliminate CD4 binding. Nat. Commun. 8, 1655 (2017).

28. Steichen, JonM. et al. HIV vaccine design to target germline precursors of glycan-dependent broadly neutralizing antibodies. Immunity $\mathbf{4 5}$ 483-496 (2016)

29. Havenar-Daughton, C., Lee, J. H. \& Crotty, S. Tfh cells and HIV bnAbs, an immunodominance model of the HIV neutralizing antibody generation problem. Immunol. Rev. 275, 49-61 (2017).

30. Pauthner, M. et al. Elicitation of robust tier 2 neutralizing antibody responses in nonhuman primates by HIV envelope trimer immunization using optimized approaches. Immunity 46, 1073-1088 (2017).

31. Drane, D., Gittleson, C., Boyle, J. \& Maraskovsky, E. ISCOMATRIX adjuvant for prophylactic and therapeutic vaccines. Expert Rev. Vaccines 6, 761-772 (2007).

32. Bianchi, M. et al. Electron-microscopy-based epitope mapping defines specificities of polyclonal antibodies elicited during HIV-1 BG505 envelope trimer immunization. Immunity 49, 288-300 (2018).

33. Egan, P. M., Belfast, M. T., Gimenez, J. A., Sitrin, R. D. \& Mancinelli, R. J. Relationship between tightness of binding and immunogenicity in an aluminum-containing adjuvant-adsorbed hepatitis B vaccine. Vaccine 27, 3175-3180 (2009).

34. Lu, F., Boutselis, I., Borch, R. F. \& Hogenesch, H. Control of antigen-binding to aluminum adjuvants and the immune response with a novel phosphonate linker. Vaccine 31, 4362-4367 (2013).

35. Kool, M. et al. Cutting edge: alum adjuvant stimulates inflammatory dendritic cells through activation of the NALP3 inflammasome. J. Immunol. 181, 3755-3759 (2008)

36. Li, H., Willingham, S., Ting, J. \& Re, F. Cutting edge: inflammasome activation by alum and alum's adjuvant effect are mediated by NLRP3. J. Immunol. 181, 17 (2008).

37. Hornung, V. et al. Silica crystals and aluminum salts activate the NALP3 inflammasome through phagosomal destabilization. Nat. Immunol. 9, 847-856 (2008).

38. Eisenbarth, S. C., Colegio, O. R., O'connor, W., Sutterwala, F. S. \& Flavell, R. A. Crucial role for the Nalp3 inflammasome in the immunostimulatory properties of aluminium adjuvants. Nature 453, 1122-1126 (2008).

39. Ramanathan, V. D., Badenoch-Jones, P. \& Turk, J. L. Complement activation by aluminium and zirconium compounds. Immunology 37, 881-888 (1979).

40. Guven, E., Duus, K., Laursen, I., Hojrup, P. \& Houen, G. Aluminum hydroxide adjuvant differentially activates the three complement

pathways with major involvement of the alternative pathway. PLoS ONE 8, e74445 (2013)

41. Flach, T. L. et al. Alum interaction with dendritic cell membrane lipids is essential for its adjuvanticity. Nat. Med. 17, 479-487 (2011).

42. Ghimire, T. R., Benson, R. A., Garside, P. \& Brewer, J. M. Alum increases antigen uptake, reduces antigen degradation and sustains antigen presentation by DCs in vitro. Immunol. Lett. 147, 55-62 (2012).

43. $\mathrm{Hu}, \mathrm{J} . \mathrm{K}$ et al. Murine antibody responses to cleaved soluble HIV-1 envelope trimers are highly restricted in specificity. J Virol. 89, 10383-10398 (2015).

Publisher's note Springer Nature remains neutral with regard to jurisdictional claims in published maps and institutional affiliations.

(C) The Author(s), under exclusive licence to Springer Nature America, Inc. 2020 


\section{Methods}

Antibodies and reagents. For spleen histology sections, tissues were stained for B220 (clone RA3-6B2; BioLegend) and CD35 (clone 8C12; BD). B220 (clone RA3-6B2; BioLegend), GL7 (clone GL7; BioLegend), CD3 (clone 17A2; BioLegend) and CD38 (clone 90; BioLegend) were used for germinal center staining. For ELISAs, anti-mouse goat IgG-HRP (Biorad) was used. For staining of VRC01 ${ }^{\text {gHL }}$ $B$ cells in the adoptive transfer model, cells were stained with antibodies against B220, GL7, CD38, CD83 (Michel-19; BioLegend), CD86 (GL-1; BioLegend), MHC II (M5/114.15.2; BioLegend), CD62L (MEL-14; BioLegend), CD95 (SA367H8; BioLegend), CD138 (281-2; BioLegend), IgD (11-26c.2a; BioLegend), CD4 (GK1.5; BioLegend), CD8- $\alpha$ (53-5.8; BioLegend), TCR- $\beta$ (H57-597; BioLegend), PD-L2 (TY25; BioLegend), CD80 (16-10A1; BioLegend), CD73 (TY/11.8; BioLegend), CD45.1 (A20; BioLegend), CD45.2 (104; BioLegend), IgG1 (RMG1-1; BioLegend), IgM (II/41; BD), Fixable Viability Dye eFluor 780 (ThermoFisher) and with biotinylated eOD-GT8 coupled to Streptavidin BV711 (BioLegend) or eOD-GT5/8 60mer conjugated to AF488, AF647 or PacificBlue (ThermoFisher). For antigen uptake measurements, Ly6C (clone HK1.4; BioLegend), CD3 (clone 17A2; BioLegend), Ly6G (clone 1A8; BioLegend), F4/80 (clone BM8; BioLegend), CD11c (N418; BioLegend), CD169 (clone 3D6.112; BioLegend) and CD11b (clone M1/70; BD) were used. Peptide-coupling reagents were purchased from Millipore Sigma (Novabiochem) unless otherwise noted. Aluminum hydroxide adjuvant (Alhydrogel) and aluminum phosphate (AdjuPhos) adjuvants were purchased from InvivoGen. Cytochrome $\mathrm{c}$ from Saccharomyces cerevisiae was purchased from Millipore Sigma.

Phosphoserine peptide synthesis. Peptides were synthesized manually by solidphase peptide synthesis. A low-loading Tentagel Rink Amide resin (Peptides International, $0.2 \mathrm{meqg}^{-1}$ ) was used for the synthesis of all peptides. Peptide couplings were performed using 4 equivalents of Fmoc-Ser (PO(OBzl)OH)-OH with 3.95 equivalents HATU (hexafluorophosphate azabenzotriazole tetramethyl uronium) for $2 \mathrm{~h}$ at $25^{\circ} \mathrm{C}$. The pSer residues were deprotected using $5 \% \mathrm{DBU}$ (1,8-diazabicyclo[5.4.0] undec-7-ene) in dimethylformamide ${ }^{44}$, while all other residues were deprotected in $20 \%$ piperidine in dimethylformamide. Double couplings were performed for the addition of any pSer monomers beyond the third. A 6-unit, Fmoc-protected oligoethylene glycol linker (Peptides International) was added following the addition of the pSer monomers to serve as a spacer between the reactive linker and pSer residues. For pSer linkers being reacted to proteins, maleoyl- $\beta$-alanine (MilliporeSigma) was coupled to the $\mathrm{N}$ terminus of the peptides. Peptides were cleaved in $95 \%$ trifluoroacetic acid, $2.5 \% \mathrm{H}_{2} \mathrm{O}$ and $2.5 \%$ triisopropylsilane, for $2.5 \mathrm{~h}$ at $25^{\circ} \mathrm{C}$. After precipitation in ice-cold diethyl ether, peptides were dried, resuspended in $0.1 \mathrm{M}$ triethylammonium acetate buffer ( $\mathrm{pH} 7$ ) and purified by HPLC on a C18 column using $0.1 \mathrm{M}$ triethylammonium acetate buffer in a gradient of acetonitrile. Peptide masses were confirmed by matrix-assisted laser desorption/ionization-time of flight mass spectrometry.

Imaging experiments using a dye-pSer ${ }_{4}$ conjugate were prepared by a $\mathrm{Cu}$-free click reaction between azide-pSer ${ }_{4}$ and a dibenzocyclooctyne (DBCO)-fluorophore, followed by HPLC purification. The azide- pSer $_{4}$ and azide-Ser ${ }_{3}-$ pSer $_{1}$ peptides were prepared using the same methods described above for the phosphoserine portion of the peptide. An oligoethylene glycol linker was not included for these linkers. At the $\mathrm{N}$ terminus of the peptide, Fmoc-5-azido-pentanoic acid (Anaspec) was used in place of the maleoyl- $\beta$-alanine and the peptide was deprotected in $20 \%$ piperidine before cleavage in trifluoroacetic acid. Cy3-DBCO (Sigma Aldrich), IR680RD-DBCO (LI-COR) and AF488-DBCO (ThermoFisher) were used for histology, LI-COR-based trafficking and flow cytometry, respectively.

Immunogen synthesis and pSer linker conjugation. eOD-GT8 gp120 and MD39 trimer antigens were synthesized as previously described ${ }^{20,27}$. Briefly, eOD with an $\mathrm{N}$-terminal cysteine was expressed in HEK cells and purified on a Nickel affinity column followed by size-exclusion chromatography on a Superdex 75 10/300 column (GE Healthcare). The eOD antigens used in Fig. 5 were prepared with the introduction of an extra I-A $\mathrm{A}^{\mathrm{b}}$-restricted helper T cell epitope from LCMV $\mathrm{gp}_{61-80}$ added at the $\mathrm{C}$ terminus of eOD. Trimer genes were synthesized and cloned into pHLsec by Genscript and then co-transfected with human furin on a pcDNA3.1 plasmid at a 2:1 trimer to furin DNA ratio using 293fectin into FreeStyle 293-F cells (ThermoFisher). Trimer supernatant was collected $5 \mathrm{~d}$ after transfection by centrifugation and purified by affinity chromatography using HisTrap HP columns (GE Healthcare) followed by size-exclusion chromatography using an S200 Increase column (GE Healthcare) in PBS at flow rate of $0.5 \mathrm{ml} \mathrm{min} \mathrm{m}^{-1}$. The molecular weight of the trimer was confirmed by size-exclusion chromatography multiangle light-scattering using DAWN HELEOS II and Optilab T-rEX instruments (Wyatt Technology).

Antigens were modified with pSer linkers using a thiol-maleimide reaction. In a solution of PBS with $1 \mathrm{mM}$ EDTA, protein antigens at $1 \mathrm{mg} \mathrm{ml}^{-1}$ were reduced for $15 \mathrm{~min}$ in 10 equivalents of tris(2-carboxyethyl)phosphine (ThermoFisher) and then tris(2-carboxyethyl)phosphine was removed by centrifugal filtration using an Amicon spin filter ( $10 \mathrm{kDa}$ MWCO). The antigens were then reacted with maleimide-pSer conjugates. For coupling to eOD and cytochrome $c$, the antigen at $1 \mathrm{mg} \mathrm{ml}^{-1}$ was reacted with 2 molar equivalents of maleimide-pSer overnight at $4{ }^{\circ} \mathrm{C}$ in PBS. MD39 timer was modified with a free cysteine at the $\mathrm{C}$-terminal end of each protomer, to which three pSer linkers could be conjugated at the base of each Env trimer. For reaction to MD39, 20 molar equivalents of maleimidepSer linker was added to $1 \mathrm{mg} \mathrm{ml}^{-1} \mathrm{MD} 39$ in PBS to ensure complete reaction to available thiols. Proteins were then separated from unreacted peptide linkers using centrifugal filters ( $10 \mathrm{kDa}$ MWCO). Reactions were initially monitored using parallel reactions with analogous linkers containing spectroscopic handles (maleimide-dibenzyocyclooctyne linkers) and coupling of the phosphoserines to the antigens was confirmed using a malachite green assay (ThermoFisher). Malachite green assays were quantified using a standard curve prepared using known concentrations of the original pSer linkers. Labeled proteins were prepared using NHS-AF647 (ThermoFisher) or NHS-IRDye 800CW (LI-COR) by reaction of 6 eq. fluorophore with eOD or MD39 $\left(1 \mathrm{mg} \mathrm{ml}^{-1}\right)$ in $50 \mathrm{mM}$ sodium carbonate buffer for $2 \mathrm{~h}$ and purified using centrifugal filtration (10 kDa MWCO). Phycoerythrin (PE)-pSer conjugates were prepared by reacting NHS-DBCO (Sigma Aldrich) heterobifunctional crosslinker to PE in sodium borate buffer ( $\mathrm{pH} 8.0)$ at varying molar ratios $(2,4,8,16$ and 32 equivalents) of linker to protein for $4 \mathrm{~h}$ at room temperature. Unreacted linkers were removed by centrifugal filtration and then DBCO-modified PE $\left(1 \mathrm{mg} \mathrm{ml}^{-1}\right)$ was split into two reactions with with 2 molar equivalents of azide-pSer ${ }_{4}$ or azide-Ser ${ }_{3} \mathrm{pSer}_{1}$ overnight at $4{ }^{\circ} \mathrm{C}$ in PBS. Unreacted pSer linkers were then removed by centrifugal filtration.

Antigen-alum binding and release. Alum-binding experiments were performed using either fluorescently labeled proteins or by ELISA. A weight ratio of 10:1 alum to protein was used for all binding experiments and immunizations, unless otherwise noted. For binding assays, protein antigen was first incubated with Alhydrogel for $30 \mathrm{~min}$ in $\mathrm{PBS}$ at $25^{\circ} \mathrm{C}$ to allow binding, then mouse serum was added for a final concentration of $10 \%(\mathrm{v} / \mathrm{v})$. Protein, alum and serum mixtures were incubated for $24 \mathrm{~h}$ at $37^{\circ} \mathrm{C}$ and solutions were centrifuged at $10,000 \mathrm{~g}$ for $10 \mathrm{~min}$ to pellet alum. The concentration of unbound protein in the supernatant was then measured by fluorescence or ELISA. Fluorescence measurements were performed using a Tecan Infinite M200 Pro absorbance/fluorescence plate reader. The fluorescence intensity was normalized to the total fluorescence of a sample that underwent the same processing but lacked alum. For ELISA measurements, 96-well Nunc MaxiSorp plates (ThermoFisher) were coated with VRC01 antibody, blocked with $1 \%$ BSA and serial dilutions of antigen were added to the plate with a maximum concentration of $2 \mu \mathrm{g} \mathrm{ml}^{-1}$. Antigen was then detected using anti-His-tag HRP (1:5,000 dilution), washed and developed with 3,3',5,5'-tetramethylbenzidine (TMB) substrate. The amount of antigen was then quantified by comparing absorbance values to control antigen with known concentrations.

B cell interactions with alum-antigen conjugates. In vitro experiments were performed using gl-VRC01-expressing Ramos B cells, kindly provided by Prof. D. Lingwood (Ragon Institute of MGH, MIT and Harvard) ${ }^{45}$. Calcium flux measurements were performed using cells loaded with $10 \mu \mathrm{M}$ Flou- 4 dye (ThermoFisher) for $30 \mathrm{~min}$ at $37^{\circ} \mathrm{C}$ in serum-free RPMI in the presence of calcium. $\mathrm{B}$ cells were washed once and heated to $37^{\circ} \mathrm{C}$ before activation. Antigen and alum were suspended in complete RPMI containing $10 \%$ FBS before addition to B cells. Baseline fluorescence was measured for $1 \mathrm{~min}$ before addition to the addition of antigen at a concentration of $1 \mu \mathrm{g} \mathrm{ml}^{-1} \mathrm{eOD}$. Alum was added to the $\mathrm{B}$ cells at $10 \mu \mathrm{g} \mathrm{ml}^{-1}$. Fluo- 4 fluorescence emission was normalized to baseline values before the addition of the antigen. For confocal and TEM imaging experiments, Ramos B cells expressing gl-VRC01 were incubated with eOD:alum formulations for $1 \mathrm{~h}$ in RPMI medium containing $10 \% \mathrm{FBS}$ at $37^{\circ} \mathrm{C}$. For confocal imaging, cells were then fixed in PBS $1 \%$ paraformaldehyde for $15 \mathrm{~min}$, washed, stained with DAPI and phalloidin, mounted on a glass slide and imaged using an inverted Olympus X71 microscope. Samples for TEM analysis were fixed in $0.1 \mathrm{M}$ sodium cacodylate buffer ( $\mathrm{pH} 7.0$ ) with $3 \%$ paraformaldehyde, $2 \%$ glutamate, $5 \%$ sucrose, pelleted and post-fixed in $1 \% \mathrm{OsO}_{4}$ in veronal-acetate buffer. The cells were stained en block overnight with $0.5 \%$ uranyl acetate in veronal-acetate buffer ( $\mathrm{pH}$ 6.0), then dehydrated and embedded in Embed-812 resin. Sections were cut on a Leica EM UC7 ultra microtome with a Diatome diamond knife at a thickness setting of $50 \mathrm{~nm}$, stained with $2 \%$ uranyl acetate and lead citrate. The sections were examined using a FEI Tecnai spirit at $80 \mathrm{KV}$ and photographed with an AMT CCD camera.

Synthesis of saponin adjuvant. For some immunizations an ISCOM-like nanoparticle consisting of self-assembled cholesterol, phospholipid and Quillaja (Quil-A) saponin was prepared as previously described ${ }^{46}$; all synthesis was performed under sterile conditions with sterile reagents. Briefly, $10 \mathrm{mg}$ each of cholesterol (Avanti Polar Lipids, 700000) and dipalmitoylphosphatidylcholine (Avanti Polar Lipids, 850355) were dissolved separately in $20 \%$ MEGA-10 (Sigma D6277) detergent at a final concentration of $20 \mathrm{mg} \mathrm{ml}^{-1}$ and $50 \mathrm{mg}$ Quil-A saponin (InvivoGen vac-quil) was dissolved in $\mathrm{MQ} \mathrm{H}_{2} \mathrm{O}$ at a final concentration of $100 \mathrm{mg} \mathrm{ml}^{-1}$. Next, dipalmitoylphosphatidylcholine solution was added to cholesterol followed by addition of Quil-A saponin in rapid succession and the volume was brought up with PBS for a final concentration of $1 \mathrm{mg} \mathrm{ml}^{-1}$ cholesterol and $2 \%$ MEGA-10. The solution was allowed to equilibrate at $25^{\circ} \mathrm{C}$ overnight, followed by $5 \mathrm{~d}$ of dialysis against PBS using a 10-k MWCO membrane. The adjuvant solution was then filter sterilized using a $0.2-\mu \mathrm{m}$ Supor syringe filter, 
concentrated using 50-k MWCO centricon filters and further purified by fast protein liquid chromatography using a Sephacryl S-500 HR size-exclusion column. Each adjuvant batch was finally characterized by negative stain TEM and dynamic light scattering (DLS) to confirm uniform morphology and size and validated for low endotoxin by Limulus Amebocyte Lystae assay (Lonza QCL-1000). Final adjuvant concentration was determined by cholesterol quantification (Sigma MAK043)

Animals and immunizations. Experiments and handling of mice were conducted under federal, state and local guidelines under an Institutional Animal Care and Use Committee approved protocol. The 6-8-week-old female BALB/c mice were purchased from the Jackson Laboratory. For imaging experiments, $10 \mu \mathrm{g}$ of eOD labeled with AF647 was injected with $100 \mu \mathrm{g}$ of alum in $100 \mu \mathrm{l}$ of PBS s.c. into the shaven tail base. Immunogenicity experiments with eOD and MD39 used immunization formulations of $5 \mu \mathrm{g}$ of antigen mixed with $50 \mu \mathrm{g}$ of alum in $100 \mu \mathrm{l}$ of PBS, unless otherwise noted. In some formulations, saponin-containing nanoparticles were injected at a concentration of $10 \mu \mathrm{g} \mathrm{ml}^{-1}$ cholesterol. Unless otherwise noted, immune responses were measured after a single primary immunization. Sera were collected by retro-orbital bleeding every other week for ELISA measurements. For rabbit immunizations, New Zealand white rabbits were immunized on days 0,56 and 112 with $100 \mu \mathrm{g}$ of MD39 and $1 \mathrm{mg}$ of alum in $1 \mathrm{ml}$ of PBS by s.c. injection (two bilateral injections of $500 \mu \mathrm{l} \mathrm{each).}$

VRC01 ${ }^{\mathrm{gHL}} \mathrm{B}$ cell adoptive transfer experiments were performed at La Jolla Institute for Immunology. All mice were between 6-10 weeks of age. Homozygous VRC01 ${ }^{\mathrm{gHL}}$ mice ${ }^{28}$ (Figs. 3 and 4 ) or heterozygous VRC01 ${ }^{\mathrm{gHL}}$ mice (Fig. 5) on a C57BL/6J (000664; Jackson Laboratory) or B6.uGFP (004353; Jackson Laboratory) background were used. B6.SJL-Ptpcra $P e p c^{b} /$ BoyJ were maintained at La Jolla Institute for Immunology. B cells were purified using CD43 MACS MicroBeads (Miltenyi Biotec) following the manufacturer's protocol. Then, $10^{3}$ (Fig. 5) or $10^{6}$ (Figs. 3 and 4) were adoptively transferred in RPMI supplemented with 1\% FCS $1-3 \mathrm{~d}$ before vaccination. For adoptive transfer of $10^{3} \mathrm{VRC} 01^{\mathrm{gHL}}$ cells, the cells were co-transferred with $5 \times 10^{5}$ wild-type (B6 or B6.SJL-Ptpcr ${ }^{a} P e p c^{b} /$ BoyJ) splenocytes to facilitate the take in the recipient mice. In some experiments, purified B cells were subsequently labeled with $5 \mu \mathrm{M}$ CellTrace Violet (Thermo Fisher) in $0.1 \%$ BSA-DPBS for $9.5 \mathrm{~min}$ at $37^{\circ} \mathrm{C}$ before adoptive transfer. For experiments in Fig. $3 a-c, f-k$ and Fig. 4, mice were i.p. vaccinated with 5-10 $\mu$ Ser $_{4}$-eOD-GT5, $\mathrm{pSer}_{8}$-eOD-GT5 or eOD-GT5 60mer adsorbed to $1 \mathrm{mg}$ of alum. For experiments in Fig. 5, LCMV gp g1-80 $_{60}$ peptide sequence was added to $\mathrm{Ser}_{4}$-eOD-GT5 and $\mathrm{pSer}_{8}$ eOD-GT5 to ensure that adequate helper T cell responses against the immunogens were induced. Transferred VRC01 $1^{\mathrm{gHL}}$ cells in the recipient spleens were identified by gating on $\mathrm{GFP}^{+} \mathrm{eOD}^{+} \mathrm{B} 220^{+}$cells (Fig. 3b,c,i,k and Fig. 4), GFP ${ }^{+} \mathrm{B} 220^{+}$cells (Fig. 5a-c), CD $45.2^{+}$CD $45.1^{-}$B222 ${ }^{+}$cells (Fig. $5 \mathrm{~d}-\mathrm{g}$ ) or $\mathrm{CTV}^{+} \mathrm{eOD}^{+} \mathrm{B}$ cells (Fig. $3 \mathrm{f}-\mathrm{h}$ ).

Envelope pseudovirus production. Envelope pseudoviruses were generated through the co-transfection of the pSG3 $\Delta$ Env backbone plasmid (obtained from the National Institutes of Health (NIH) AIDS Research and Reference Reagent Program, Division of AIDS, NIAID, NIH $)^{47,48}$ and a plasmid encoding the full Env gp160 in a 3:1 ratio in HEK293T cells (American Type Culture Collection) using the PEIMAX transfection reagent (Polysciences). Following $48 \mathrm{~h}$, the medium was filtered through a $0.45-\mu \mathrm{m}$ Steriflip unit (EMD Millipore), aliquoted, frozen and titrated.

Neutralization assay. The neutralization assay ${ }^{49}$ was performed with DMEM (Corning) supplemented with 10\% FBS (Corning), 1\% L-glutamine (Corning), $0.5 \%$ gentamicin (Sigma) and 2.5\% HEPES (Gibco) and all incubations were performed at $37^{\circ} \mathrm{C}$ with $5 \% \mathrm{CO}_{2}$. On day $1,25 \mu$ of diluted pseudovirus mixed with $25 \mu \mathrm{l}$ of 1:3 serially diluted serum or control antibody was incubated for $1 \mathrm{~h}$, followed by the addition of $20 \mu \mathrm{l}$ of TZM-bl cells at a concentration of 500,000 cells $\mathrm{ml}^{-1}$ with diethylaminoethyl-dextran at a final concentration of $40 \mu \mathrm{g} \mathrm{ml}^{-1}$ These were incubated for $24 \mathrm{~h}$ and on day $2,130 \mu \mathrm{l}$ of fresh DMEM was added and the samples, which were once again incubated overnight. Finally, on day 3 , the medium was completely removed and $60 \mu \mathrm{l}$ of lysis buffer together with $60 \mu \mathrm{l}$ of luciferase substrate (Promega) was added per well and luminescence was measured on the Synergy2 plate reader (BioTek). Neutralization data are reported as ID $_{50}$ or median inhibitory concentration $\left(\mu \mathrm{g} \mathrm{ml}^{-1}\right)$ values that were calculated as the dilution or concentration at which a $50 \%$ reduction in infection was observed. Neutralization assays were performed in triplicate and s.e.m. was reported. The TZM-bl cell line, engineered from CXCR4-positive HeLa cells to express CD4, CCR5 and a firefly luciferase reporter gene (under control of the HIV-1 LTR) was obtained from the NIH AIDS Research and Reference Reagent Program, Division

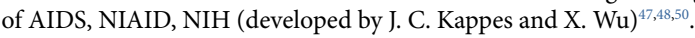

Whole-mouse imaging and lymph node trafficking. Signals of antigens labeled with AF647 were measured using an IVIS fluorescence imaging system over time at the injection site. Total radiance was normalized to the initial IVIS signal at day 0 , measured $30 \mathrm{~min}$ after injection. To simultaneously track eOD and alum, we synthesized an infrared dye (IR680)- pSer $_{4}$ conjugate (Extended Data Fig. 4a). IR680-pSer ${ }_{4}$ bound very tightly to alum, with minimal detectable dye released from alum following $72 \mathrm{~h}$ incubation in serum (Extended Data Fig. $4 \mathrm{~b}$ ). We then mixed alum with an equimolar amount of pSer $_{8}$-eOD (labeled with IR800 dye) and IR680 $\mathrm{pSer}_{4}$ or alum mixed with dye-labeled $\mathrm{Ser}_{4}$-eOD and IR680-pSer ${ }_{4}$ as a control, and immunized mice. For these experiments, alum $(50 \mu \mathrm{g})$ was labeled by addition of a mixture of $0.5 \mathrm{nmol} \mathrm{pSer}$-IR680 and $5 \mu \mathrm{g}$ IR800 dye-labeled eOD. The alum:eOD samples were mixed for $30 \mathrm{~min}$ at $25^{\circ} \mathrm{C}$ and then injected s.c. into groups of $\mathrm{BALB} / \mathrm{c}$ mice. Inguinal LNs were excised at various time points and whole-tissue fluorescence was measured by a LI-COR Odyssey fluorescence imaging system at $700 \mathrm{~nm}$ and $800 \mathrm{~nm}$ wavelengths. Values represent the integrated fluorescence intensity. For histology experiments, mice were immunized by s.c. or i.p. injections with $10 \mu \mathrm{g}$ of AF647-labeled antigen labeled and $100 \mu \mathrm{g}$ of alum.

Immunohistochemistry of injection sites and spleens. Mice were immunized by s.c. or i.p. injections with $10 \mu \mathrm{g}$ of AF647-labeled antigen and $100 \mu \mathrm{g}$ of alum. The i.p. injections also included $\mathrm{Cy} 3-\mathrm{pSer}_{4}$ to label alum during imaging. Histology sections were prepared using skin from the injection site, spleen or LN tissues that were fixed with $4 \%$ paraformaldehyde overnight, washed and embedded in a $3 \mathrm{wt} \%$ low melting point agarose at $37^{\circ} \mathrm{C}$ then allowed to cool and solidify on ice for $15 \mathrm{~min}$. The $100-200-\mu \mathrm{m}$ sections were prepared using a Vibratome (Leica VT1000S) and suspended in ice-cold PBS then transferred into a blocking solution containing $10 \%$ goat serum, $0.2 \%$ Triton-X100 and $0.05 \%$ sodium azide overnight at $37^{\circ} \mathrm{C}$ before immunostaining. For the injection site sections, slices were stained in a solution of morin $(1 \mu \mathrm{M})$ in $0.5 \%$ acetic acid in ethanol. For spleen sections, the tissues were stained for B220 (clone RA3-6B2, BioLegend) and CD35 (clone $8 \mathrm{C} 12, \mathrm{BD}$ ). Antibodies were used at 1:100 dilution in blocking buffer overnight at $37^{\circ} \mathrm{C}$, followed by washes with PBS $0.05 \%$ Tween and mounted on a glass slide with ProLong Diamond antifade mounting medium (Life Technologies). Images were acquired using a Leica SP8 laser scanning confocal microscope with a $\times 10$ or $\times 25$ water objective. Images were processed with Fiji software.

Inductively coupled plasma mass spectrometry of aluminum in LNs. Inductively coupled plasma mass spectrometry (ICP-MS) measurements were performed using immunizations of $200 \mu \mathrm{g}$ of alum mixed with eOD or $\mathrm{pSer}_{4}$-eOD. Inguinal LNs were excised at indicated timepoints, dissolved in nitric acid, digested at $200^{\circ} \mathrm{C}$ for $20 \mathrm{~min}$ in a Milestone UltraWave microwave digestion system, diluted into water to $2 \%$ nitric acid and analyzed by an Agilent 7900 ICP-MS. A standard curve of aluminum from $1 \mu \mathrm{g} \mathrm{ml}^{-1}$ to $1 \mathrm{ng} \mathrm{ml}^{-1}$ was used to quantify the samples, along with an internal standard of rhodium for every sample.

ELISA analysis of antibody titers. For ELISAs to measure anti-eOD titers, Nunc MaxiSorp plates were directly coated with unmodified, monomeric eOD $\left(1 \mu \mathrm{g} \mathrm{ml}^{-1}\right)$ and blocked with PBS containing 1\% BSA. For anti-MD39 titer ELISAs, plates were coated with an ST-II antibody $\left(2 \mu \mathrm{g} \mathrm{ml}^{-1}\right)$ and MD39 containing an ST-II tag was added to the plates after blocking with 1\% BSA in PBS. Responses against MD39 gp120 were measured by coating ELISA plates with a rabbit antiHis-tag antibody (Genscript), followed by blocking and then addition of his-tagged MD39 gp120 antigen $\left(2 \mu \mathrm{g} \mathrm{ml}^{-1}\right)$. Serial dilutions of sera were added to the blocked plates for $2 \mathrm{~h}$ at $25^{\circ} \mathrm{C}$, washed in PBS with $0.05 \%$ Tween- 20 and incubated with anti-mouse IgG-HRP (BioRad). ELISA plates were developed with TMB substrate and absorbance values at $450 \mathrm{~nm}$ were measured. Base-blocking ELISAs were performed using plates coated with human VRC01 $\left(2 \mu \mathrm{g} \mathrm{ml}^{-1}\right)$, followed by a blocking step with $1 \%$ BSA in PBS and the addition of MD39 $\left(2 \mu \mathrm{g} \mathrm{ml}^{-1}\right)$ for $2 \mathrm{~h}$ at $25^{\circ} \mathrm{C}$. To obscure the base of trimer, $20 \mu \mathrm{g} \mathrm{ml}^{-1}$ base-binding antibody was added for $30 \mathrm{~min}$ before the addition of serum dilutions. Serum dilutions were added directly to the wells with the base-binding antibody still remaining and absorbance values were compared to wells that were incubated with 1\% BSA in PBS instead of the base-binding antibody. His-tag-specific antibodies were measured using plates coated in streptavidin $\left(2 \mu \mathrm{g} \mathrm{ml}^{-1}\right)$ followed by addition of biotin-H6 peptide (Genscript). Titers were determined at an absorbance cutoff of $0.1 \mathrm{OD}$ and $0.3 \mathrm{OD}$ for MD39 and eOD immunizations, respectively.

Antigenicity of trimer bound to alum. Antigenicity of MD39 trimer bound to alum was measured using a modified sandwich ELISA protocol. An irrelevant protein (cytochrome c from S. cerevisiae, Sigma Aldrich) modified with a $\mathrm{pSer}_{4}$ linker was first coated on ELISA plates overnight. Alum at $100 \mu \mathrm{g} \mathrm{ml}^{-1}$ was added to the plates to bind to the exposed pSer residues present on cytochrome $\mathrm{c}$. Control wells were coated with mouse VRC01 at $2 \mu \mathrm{g} \mathrm{ml}^{-1}$. Plates were washed with PBS containing $0.05 \%$ Tween- 20 and then incubated with solutions of $1 \%$ BSA containing MD39-pSer $\left(1 \mu \mathrm{g} \mathrm{ml}^{-1}\right)$ for $2 \mathrm{~h}$ at $25^{\circ} \mathrm{C}$. The bnAbs or base-specific antibodies were added at $100 \mathrm{ng} \mathrm{ml}^{-1}$, and detected with a secondary Ab-HRP conjugate, followed by development in TMB substrate.

ELISPOT and germinal center analysis. Bone marrow ELISPOTs were performed 3 months after immunization according to manufacturer's instructions, unless otherwise noted (MabTech). ELISPOT plates were coated with an anti-mouse IgG antibody and isolated bone marrow cells were added to the plate for $4 \mathrm{~h}$ at $37^{\circ} \mathrm{C}$ in complete RPMI (500,000 or 100,000 cells per well for antigen-specific or total IgG responses, respectively). After washing the plates, antigen-specific responses were measured by the addition of biotinylated antigen $\left(1 \mu \mathrm{g} \mathrm{ml}^{-1}\right)$ for $2 \mathrm{~h}$ at $25^{\circ} \mathrm{C}$, followed by streptavidin-HRP. Bone marrow cells isolated from each mouse were 
measured in triplicate. For measurement of germinal center responses, mice were killed $9 \mathrm{~d}$ after immunization and LNs were mechanically digested and passed through a $70-\mu \mathrm{m}$ filter. Cells were then stained with antibodies against CD3e, B220, CD38 and GL7, as well as an AF647-labeled eOD-60mer nanoparticle ${ }^{20}$, to identify eOD-specific B cells. Flow cytometric analysis was performed using a BD Canto.

Single-cell RNA-seq. Full-length RNA-seq from single cells was performed using Smart-seq2 methods ${ }^{51}$ with some modifications as previously described ${ }^{52}$. Single VRC01 ${ }^{\text {gHL }} \mathrm{B}$ cells $\left(\mathrm{B} 220^{+} \mathrm{GFP}{ }^{+} \mathrm{eOD}-\mathrm{BV} 711^{+} \mathrm{AF} 647^{+/-}\right.$live singlets) were sorted by a FACSAria III (BD Bioscience) fitted with a $70-\mu \mathrm{m}$ nozzle and run at 52 psi. Single cells were sorted using 'single-cell' precision mode and directly collected into 96-well PCR plates containing RNA lysis buffer with RNAse inhibitor (Takara). A CDU chiller was used for sorting and set to $5^{\circ} \mathrm{C}$ to ensure RNA recovery. RNA loss was minimized by on-plate reverse transcription and whole-transcriptome preamplification PCR (23 cycles). PCR preamplified product was purified twice using $0.8 \times$ AMPure XP beads (Beckman Coulter) to remove any trace of primerdimers. Samples that failed standard quality control steps, as previously described ${ }^{51}$, were removed from further downstream steps. Barcoded Illumina sequencing libraries were generated from $0.3-0.5 \mathrm{ng}$ of preamplified cDNA using the tn 5 DNA insertion method (Nextera XT library preparation kit, Illumina). Libraries were sequenced on the HiSeq2500 Illumina platform to obtain single-end 50-bp reads. The reads were aligned to the mouse mm10 reference genome using TopHat (v.1.4.1) $)^{53}$. Sequencing read coverage per gene was counted using HTSeq-count. Counts per gene were obtained by counting all the transcripts mapping to a gene.

Single-cell differential gene expression analysis. Unbiased clustering of single cells (Supplementary Fig. 5C) was performed using Seurat (v.2.3.4) $)^{54}$. Only VRC01 ${ }^{\mathrm{gHL}} \mathrm{B}$ cells with 500-2,500 expressed genes and $5 \times 10^{5}$ to $2 \times 10^{7}$ total read count and only genes expressed in at least two cells were included in the analysis. We applied the default Seurat global-scaling normalization method, where gene expression measurements for each cell are normalized by the total expression, multiplied by $10^{4}$ and natural log-transformed. The number of reads that mapped to each cell was regressed out before scaling and centering data for dimensionality reduction. Principal-component analysis was performed on 791 variable genes and then dimensionality reduction was performed using a $t$-distributed stochastic neighbor embedding algorithm employing the top 20 principal components. Differentially expressed genes were identified by performing pairwise comparisons using MAST; ${ }^{55}$ within the Seurat R package. VRC01 ${ }^{\mathrm{gHL}} \mathrm{B}$ cells from each group were compared against the cells from the remaining groups using a 1.25-fold cutoff and adjusted $P$ values $<0.05$.

Bulk RNA-seq, mapping and analysis. A total of 5,000-10,000 VRC01 ${ }^{\mathrm{gHL}} \mathrm{B}$ cells $\left(\mathrm{B} 220^{+} \mathrm{GFP}^{+} \mathrm{eOD}-\mathrm{BV} 711^{+} \mathrm{AF} 647^{+/-}\right.$live singlets) were sorted by a FACSAria III into Trizol LS (Invitrogen). RNA extraction was performed using miRNeasy micro kits (Qiagen) for downstream RNA-Smart-Seq2 input requirements ${ }^{51,52}$. The reads were aligned to the mouse mm 10 reference genome. Raw counts were imported to R/Bioconductor package DESeq2 (v.3.1). Differentially expressed genes were identified using DESeq2 pairwise comparisons.

Gene set enrichment analysis. GSEA ${ }^{56}$ was performed to explore whether specific gene signatures were enriched between $\mathrm{VRC} 01^{\mathrm{gHL}} \mathrm{B}$ cells from two groups. Preranked gene lists were generated based on $\log _{10}$-transformed nominal $P$ values obtained from DeSeq2 analysis. Metabolism-related Hallmark gene sets (E2F targets (M5925); glycolysis (M5937); mTorc1 targets (M5924); Myc-targets v.1 (M5926); oxidative phosphorylation (M5936) $)^{57}$ and Myc downregulated targets (M3923) ${ }^{58}$ were obtained from the Molecular Signatures Database. Additionally, BCR upregulated and downregulated genes, CD40 upregulated and downregulated genes, TLR4 upregulated genes and TLR9 upregulated genes were custom curated on the basis of the work of Zhu et al. ${ }^{59}$. Since BCR, CD40 and TLRs regulate common downstream genes ${ }^{59}, \mathrm{CD} 40$-regulated genes were removed from BCR upregulated and downregulated gene sets to create more selective signature gene sets. Likewise, BCR-regulated genes were removed from CD40 upregulated and downregulated gene sets and TLR4 or TLR9 upregulated genes sets. Components of antigen processing through MHC II processing were custom curated on the basis of the work of Lee et al. ${ }^{60}$.

BCR sequencing. Single VRC $01^{\mathrm{gHL}} \mathrm{B}$ cells were sorted on $\mathrm{d} 30$ and $\mathrm{d} 35$ post pSereOD-GT5:alum immunization on a FACSAria Fusion (BD Biosciences) fitted with a $70-\mu \mathrm{m}$ nozzle and run at $61.5 \mathrm{psi}$. Single cells were sorted using 'purity' precision mode. The flow rate was kept at 2.4 or lower to ensure high-quality cell sorting. A $\mathrm{CDU}$ chiller was used for sorting and set to $5^{\circ} \mathrm{C}$ to ensure RNA recovery. IgG1 ${ }^{+}$ GC VRC01 $1^{\mathrm{gHL}} \mathrm{B}$ cells were defined as singlet, scatter, live, $\mathrm{CD} 4^{-}, \mathrm{CD}^{-}, \mathrm{CD} 138^{-}$ $\mathrm{B} 220^{+} \mathrm{GL}^{+}, \mathrm{CD}^{2} 8^{-}, \mathrm{IgD}^{-}, \mathrm{CD} 45.1^{-}, \mathrm{CD} 45.2^{+}, \mathrm{IgG1}^{+}$and $\mathrm{IgM}^{-}$. VRC01 ${ }^{\mathrm{gHL}}$ memory $\mathrm{B}$ cells were defined as singlet, scatter, live, $\mathrm{CD}^{-}, \mathrm{CD}^{-}, \mathrm{CD} 138^{-}, \mathrm{B} 220^{+}, \mathrm{GL}^{-}$, $\mathrm{CD}^{2} 8^{+}, \mathrm{IgD}^{-} \mathrm{CD} 45.1^{-}, \mathrm{CD} 45.2^{+}, \mathrm{IgG1}^{+}$and $\mathrm{IgM}^{-}$. Naive VRC01 ${ }^{\mathrm{gHL}} \mathrm{B}$ cells (gated as singlet, scatter, live, TCR- $\beta^{-}, \mathrm{B} 220^{+}, \mathrm{GL}^{-}$and $\left.\mathrm{eOD}-\mathrm{GT}^{+}\right)$were sorted from an unimmunized mouse using 'single-cell' precision mode on a FACSAria II. Cells were sorted directly into $15 \mu \mathrm{l}$ of lysis buffer $(0.1 \mathrm{M}$ Trizma $\mathrm{HCl}$ (pH 8), Sigma), containing $10 \mu \mathrm{g} \mathrm{ml}^{-1}$ Poly(A) (Roche) and $500 \mathrm{U} \mathrm{ml}^{-1}$ Murine RNase inhibitor (New England BioLabs). First-strand cDNA synthesis was performed using
SuperScript II Reverse Transcriptase (Invitrogen). VRC01 heavy chain gene amplification from IgG1 ${ }^{+}$VRC01gHL B cells was performed by nested $\mathrm{PCR}$ reaction as previously described ${ }^{24}$. For naive $\mathrm{IgM}^{+} \mathrm{VRC} 01^{\mathrm{gHL}} \mathrm{B}$ cells, the first of the nested PCR was performed using msVHE forward primer (5' GAGGTGCAGCTGCAGGAGTCTGG) and a murine IgM-specific reverse primer ( $5^{\prime}$ TGGGAAGGTTCTGATACCCTGGATG $)^{61}$, followed by the second reaction using a VRC01 heavy chain-specific forward primer (5' GTGCAGCTGGTGCAGTCTGGGGC) and a reverse primer

(5' GACCTGGAGAGGCCATTCTTACC). Sanger sequencing of the PCR products was performed using the forward and reverse primers from the second PCR reaction. Reads were quality checked, trimmed, aligned and analyzed using Sequencher (v.5.1). Amino acid mutation assignments were made using Kabat numbering.

Antibody isolation and EM. IgG was purified from serum with protein A and then digested into Fab with papain. Purified Fab was added to BG505 SOSIP D664 MD39 mC trimer and complexes were purified using a GE Superose 6 column (GE HealthCare). Complexes were added to 400 mesh carbon-coated grids and stained with $2 \%$ uranyl formate. Grids of complexes made of Fab from serum collected at week 10 post immunization were imaged on a Tecnai F20 at $200 \mathrm{KeV}$ using a $4 \mathrm{k} \times 4 \mathrm{k}$ Gatan Ultrascan 4000 CCD. Complexes from week 18 post immunization were collected on a Tecnai Spirit at $120 \mathrm{KeV}$ using a $4 \mathrm{k} \times 4 \mathrm{k}$ TemCam F416 camera. Micrographs were collected with Leginon ${ }^{62}$ and processed with Appion ${ }^{63}$. Particles were chosen using DoGpicker ${ }^{64}$ and stacked, and initial 2D classes were made with MSA/MRA ${ }^{65}$. After removing classes with double particles or those not resembling trimers or protomers, unbinned particles were put into Relion ${ }^{66}$, where 3D classification was performed to obtain maps of trimer and Fab complexes. Clean maps were then refined and put into Chimera ${ }^{67}$ to segment and compare.

Statistics. Statistics were analyzed using GraphPad Prism software. All graphs represent mean and standard deviations unless otherwise noted. Comparisons of more than two groups were performed using a one-way ANOVA with a Tukey post hoc test to determine statistical significance. For comparisons of experiments with only two groups, two-tailed unpaired Student's $t$-tests were used. Fisher's exact test was performed to determine statistical significance of BCR mutations compared to naive B cells.

Reporting Summary. Further information on research design is available in the Nature Research Reporting Summary linked to this article.

\section{Data availability}

All requests for raw and analyzed data and materials are promptly reviewed by the MIT Technology Licensing Office to verify whether the request is subject to any intellectual property or confidentiality obligations. Any data and materials that can be shared will be released via a Material Transfer Agreement.

\section{References}

44. Attard, T. J., O’Brien-Simpson, N. M. \& Reynolds, E. C. Identification and suppression of $\beta$-elimination byproducts arising from the use of Fmoc-Ser(PO3Bzl,H)-OH in peptide synthesis. Int. J. Pept. Res. Ther. 15, 69-79 (2009).

45. Weaver, G. C. et al. In vitro reconstitution of B cell receptor-antigen interactions to evaluate potential vaccine candidates. Nat. Protoc. 11, 193 (2016).

46. Lövgren-Bengtsson, K. \& Morein, B. in Methods in Molecular Medicine, Vaccine Adjuvants: Preparation Methods and Research Protocols, Vol. 42 (ed. O'Hagan, D.) 239-258 (Humana Press, 2000).

47. Wei, X. et al. Emergence of resistant human immunodeficiency virus type 1 in patients receiving fusion inhibitor (T-20) monotherapy. Antimicrob. Agents Chemother. 46, 1896-1905 (2002).

48. Wei, X. et al. Antibody neutralization and escape by HIV-1. Nature 422, 307-312 (2003)

49. Sarzotti-Kelsoe, M. et al. Optimization and validation of the TZM-bl assay for standardized assessments of neutralizing antibodies against HIV-1. J. Immunol. Methods 409, 131-146 (2014).

50. Platt, E. J., Wehrly, K., Kuhmann, S. E., Chesebro, B. \& Kabat, D. Effects of CCR5 and CD4 cell surface concentrations on infections by macrophagetropic isolates of human immunodeficiency virus type 1. J. Virol. 72, 2855-2864 (1998)

51. Picelli, S. et al. Full-length RNA-seq from single cells using Smart-seq2. Nat. Protoc. 9, 171 (2014).

52. Rosales, S. L. et al. in Type 2 Immunity (ed. Reinhardt, R. L.) 275-301 (Springer, 2018).

53. Trapnell, C., Pachter, L. \& Salzberg, S. L. TopHat: discovering splice junctions with RNA-Seq. Bioinformatics 25, 1105-1111 (2009).

54. Butler, A., Hoffman, P., Smibert, P., Papalexi, E. \& Satija, R. Integrating single-cell transcriptomic data across different conditions, technologies, and species. Nat. Biotechnol. 36, 411 (2018). 
55. Finak, G. et al. MAST: a flexible statistical framework for assessing transcriptional changes and characterizing heterogeneity in single-cell RNA sequencing data. Genome Biol. 16, 278 (2015).

56. Subramanian, A. et al. Gene set enrichment analysis: a knowledge-based approach for interpreting genome-wide expression profiles. Proc. Natl Acad. Sci. USA 102, 15545-15550 (2005).

57. Liberzon, A. et al. The molecular signatures database Hallmark gene set collection. Cell Syst. 1, 417-425 (2015)

58. Yu, D., Cozma, D., Park, A. \& Thomas-Tikhonenko, A. Functional Validation of genes implicated in lymphomagenesis: an in vivo selection assay using a Myc-induced B cell tumor. Ann. NY Acad. Sci. 1059, 145-159 (2005).

59. Zhu, X. et al. Analysis of the major patterns of B cell gene expression changes in response to short-term stimulation with 33 single ligands. J. Immunol. 173, 7141-7149 (2004)

60. Lee, J. A. et al. Components of the antigen processing and presentation pathway revealed by gene expression microarray analysis following B cell antigen receptor (BCR) stimulation. BMC Bioinformatics 7, 237 (2006).

61. Busse, C. E., Czogiel, I., Braun, P., Arndt, P. F. \& Wardemann, H. Single-cell based high-throughput sequencing of full-length immunoglobulin heavy and light chain genes. Eur. J. Immunol. 44, 597-603 (2014).

62. Potter, C.S. et al. Leginon: a system for fully automated acquisition of 1000 electron micrographs a day. Ultramicroscopy 77, 153-161 (1999).

63. Lander, G.C. et al. Appion: An integrated, database-driven pipeline to facilitate EM image processing. J. Struct. Biol. 166, 95-102 (2009).

64. Voss, N.R., Yoshioka, C.K., Radermacher, M., Potter, C.S. \& Carragher, B. DoG Picker and TiltPicker: Software tools to facilitate particle selection in single particle electronmicroscopy. J. Struct. Biol. 166, 205-213 (2009).

65. Zhao, Z. \& Singer, A. Rotationally invariant image representation for viewing direction classification in cryo-EM. J. Struct.Biol. 186, 153-166 (2014).

66. Scheres, S.H.W. RELION: Implementation of a Bayesian approach to cryo-EM structure determination. J. Struct. Biol. 180, 519-530 (2012).

67. Pettersen, E.F., et al. UCSF Chimera-Avisualization system for exploratory research and analysis. J. Comput. Chem. 25, 1605-1612 (2004).

\section{Acknowledgements}

This work was supported in part by the NIAID under awards UM1AI100663 and

UM1AI144462 (to D.J.I., W.R.S., S.C. and D.R.B.), AI125068 (to S.C. and D.J.I.),
AI048240 (to D.J.I.), K99AI145762 (to R.K.A.) and AI113867 (to W.R.S.), the Koch Institute Support (core) grant P30-CA14051 from the National Cancer Institute, the Ragon Institute of MGH, MIT and Harvard and by the International AIDS Vaccine Initiative (IAVI) Neutralizing Antibody Consortium (NAC) and Center (to D.R.B., A.B.W. and W.R.S.); and through the Collaboration for AIDS Vaccine Discovery funding for the IAVI NAC Center (to D.R.B., A.B.W. and W.R.S.). The content is solely the responsibility of the authors and does not necessarily represent the official views of the National Institutes of Health. D.J.I. is an investigator of the Howard Hughes Medical Institute.

\section{Author contributions}

T.J.M., D.J.I., Y.K. and S.C. planned and designed experiments and analyzed data. W.R.S. D.W.K. and S.M. designed and produced immunogens. T.J.M., W.A., J.Y.H.C., N.W., H.A.S., S.H.-D.L.M., S.L., G.S., R.K.A., M.B.M., N.L. and Y.A. performed experiments. J.N.B. and D.R.B. carried out neutralization analysis of rabbit sera. H.L.T. and A.B.W. performed TEM analysis of rabbit sera. T.J.M., D.J.I., W.R.S., Y.K. and S.C. wrote the manuscript. T.J.M., D.J.I., Y.K. and S.C. conceptualized the project. D.J.I. and S.C. provided research supervision.

\section{Competing interests}

D.J.I., T.J.M., Y.K., S.C. and W.R.S. are named as inventors on patent applications filed by MIT and The Scripps Research Institute related to the data presented in this work.

\section{Additional information}

Extended data is available for this paper at https://doi.org/10.1038/s41591-020-0753-3. Supplementary information is available for this paper at https://doi.org/10.1038/ s41591-020-0753-3.

Correspondence and requests for materials should be addressed to D.J.I.

Peer review information Saheli Sadanand was the primary editor on this article and managed its editorial process and peer review in collaboration with the rest of the editorial team.

Reprints and permissions information is available at www.nature.com/reprints. 
a

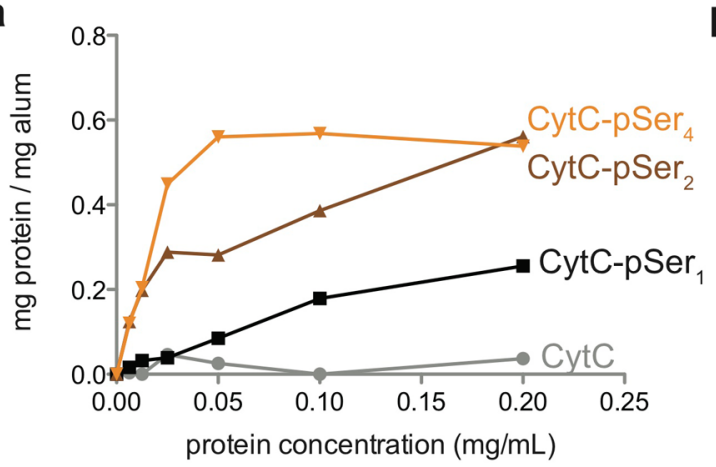

C

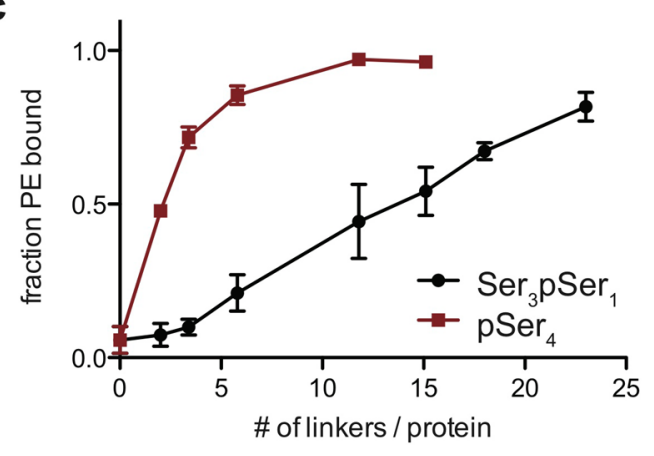

b

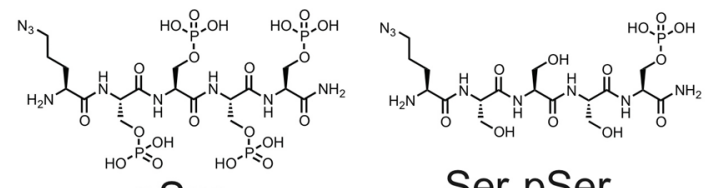

$\mathrm{pSer}_{4}$

$\operatorname{Ser}_{3} \mathrm{pSer}_{1}$ d

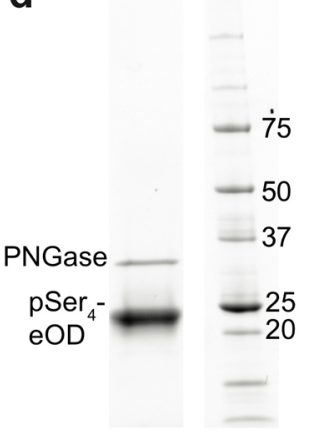

f

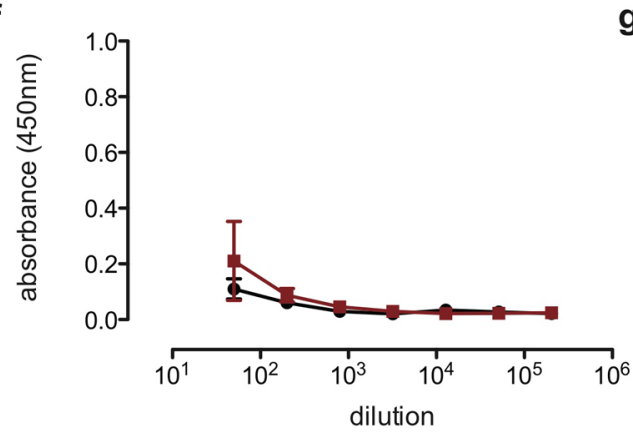

g

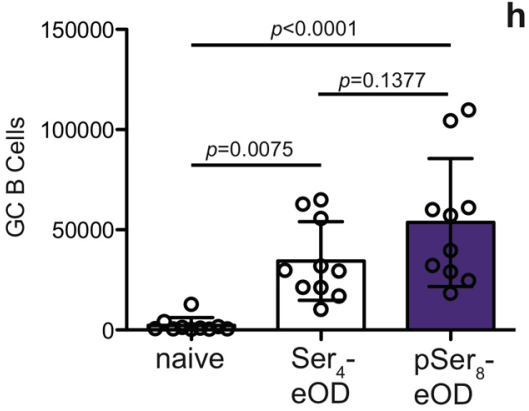

e

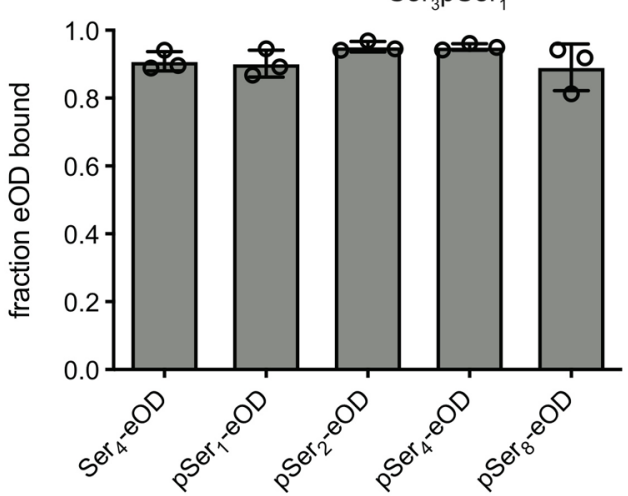

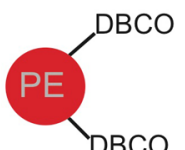

$\underset{\mathrm{N}_{3}-\mathrm{pSer}_{4}}{\mathrm{PE}} \mathrm{pSer}_{4}$

or

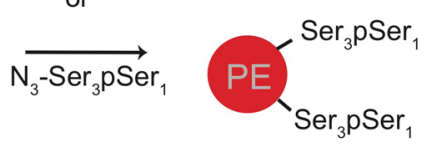

h

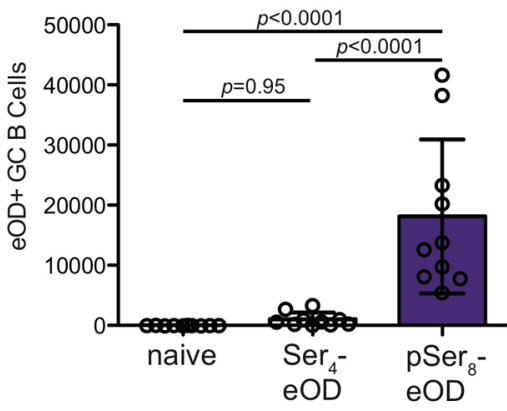

Extended Data Fig. 1 | Phosphoserines mediate protein binding to alum and influence immune response. (a) Unmodified cytochrome- $\mathrm{C}$ protein or an equivalent concentration of cytochrome- $\mathrm{C}$ conjugated with a single $\mathrm{pSer}$ linker containing 1, 2, or 4 phosphoserine groups was mixed with $100 \mu \mathrm{gg} / \mathrm{mL}$ Alhydrogel in $10 \mathrm{mM}$ MOPS buffer. Alum was separated by centrifugation and bound protein was detected using absorbance at $410 \mathrm{~nm}$ compared to a standard curve of known protein concentration. (b) Peptide linkers of equivalent molecular size were prepared, composed of an azide functional group linked to either 4 phosphoserines, or 3 serines and 1 phosphoserine. These linkers were coupled to DBCO-modified phycoerythrin (PE) via copper-free click chemistry at a range of total linkers per protein. (c) pSer/Ser-conjugated PE ( $5 \mu \mathrm{g} / \mathrm{mL})$ with varying numbers of linkers per protein was mixed with alhydrogel $(50 \mu \mathrm{g} / \mathrm{mL})$ for $30 \mathrm{~min}$, followed by addition of mouse serum (final concentration $10 \%$ vol/vol) for 24 hr. Protein bound to alum after this incubation was assessed by fluorescence spectroscopy. ( $n=3$ samples/group). Data represents mean \pm SD. (d) PAGE gel of eOD protein reacted with $\mathrm{pSer}_{4}-\mathrm{eOD}$. PNGase treatment was used with eOD to remove glycans prior to running gel. Representative gel from two experiments. Gel image is cropped. (e) eOD proteins $(10 \mu \mathrm{g} / \mathrm{mL})$ conjugated with a single 4-residue serine linker, or pSer linkers containing 1-8 phosphoserines, were incubated with AlHydrogel $(100 \mu \mathrm{g} / \mathrm{mL})$ for $30 \mathrm{~min}$ in TBS at $25^{\circ} \mathrm{C}$, followed by measurement of bound protein by fluorescence. ( $n=3$ samples/group). Center lines represent mean and error bars represent SD. (f) Day 63 sera from BALB/c mice ( $n=5$ animals/group) immunized with eOD (black) or pSer ${ }_{4}$-eOD (red) were tested for binding to $\mathrm{pSer}$ tags. Plates were coated with $\mathrm{pSer}_{4}$-cytochrome $\mathrm{c}$ to quantify the pSer-specific response. Data represents mean \pm SD. $(\mathbf{g}, \mathbf{h})$ Germinal center B cells isolated from the inquinal lymph nodes of BALB/c mice ( $n=10$ animals/group, pooled from 2 experiments) 9 days after immunization with eOD variants and Al-Hydrogel. Cell counts are provided for GC B cells ( $g$ ) and eOD-specific GC B cells ( $h$ ). Center lines represent mean and error bars represent SD. Statistical tests were performed using one-way ANOVA with Tukey's post test. 
a

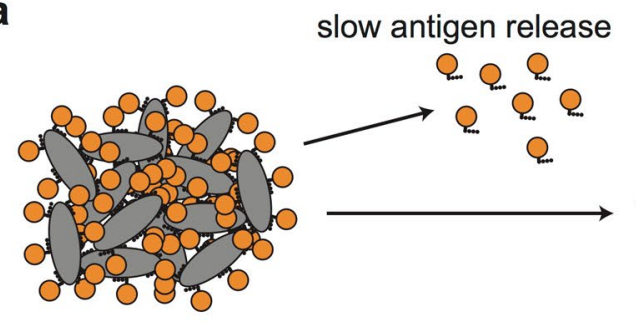

b

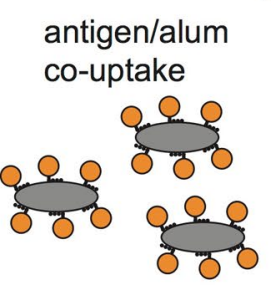

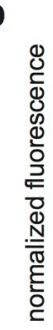

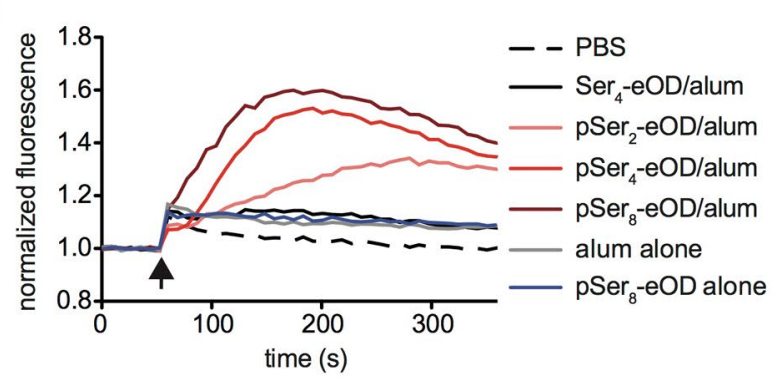

\section{C}

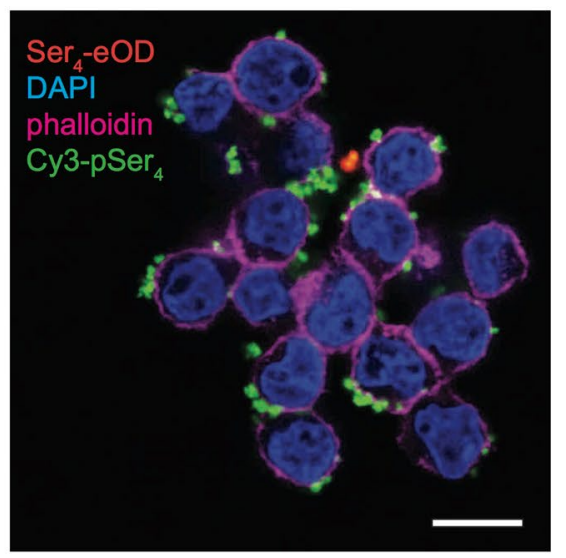

d

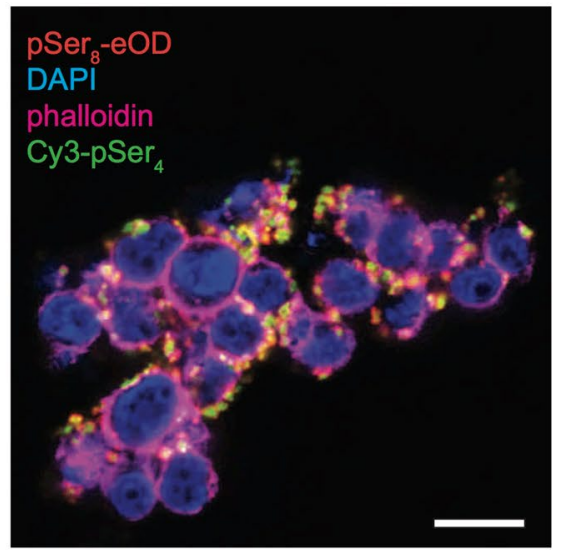

Extended Data Fig. 2 | Antigen-specific B cells internalize pSer-antigens bound to alum particles in vitro. (a) Schematic of potential release of free antigen vs. release of antigen-decorated alum particles at the injection site. (b) gIVRC01-expressing human B cells were mixed with eOD (50 nM) and alum $(10 \mu \mathrm{g} / \mathrm{mL}$ ), or alum alone $(10 \mu \mathrm{g} / \mathrm{mL})$. Shown is calcium signaling in B cells following addition of antigen/alum at $60 \mathrm{sec}$ (arrowhead) by the Fluo-4 fluorescence reporter. (c, d) Alum was labeled by mixing with Cy3-pSer ${ }_{4}$. gIVRC01-expressing B cells were then incubated with fluorescent eOD (50 nM) and fluorophore-tagged alum $(10 \mu \mathrm{g} / \mathrm{mL})$ for 1 hour, and then imaged by confocal microscopy. (scale bars $=10 \mu \mathrm{m}$ ). Experiment was performed in two times, showing representative images from one experiment. 
a

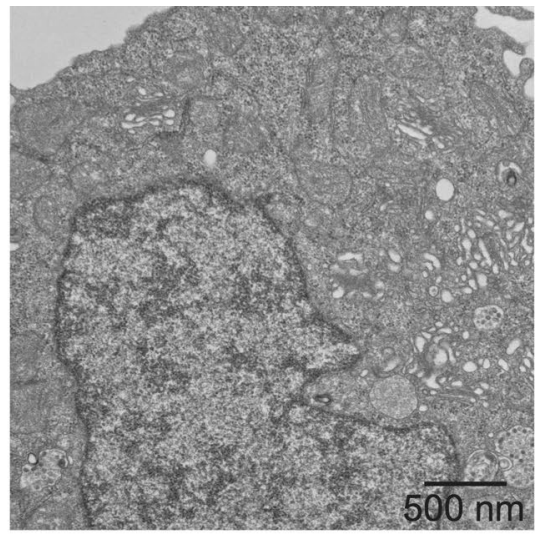

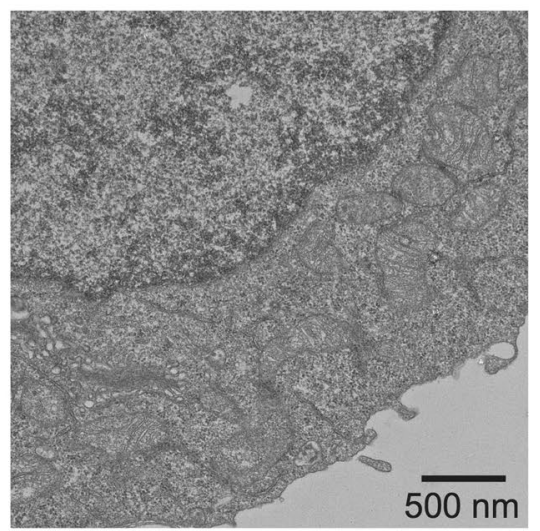

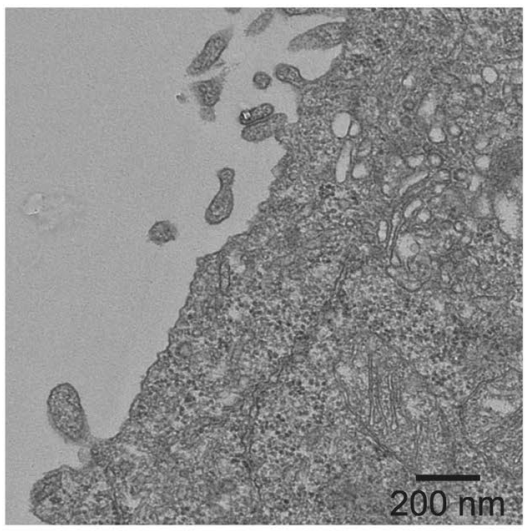

b
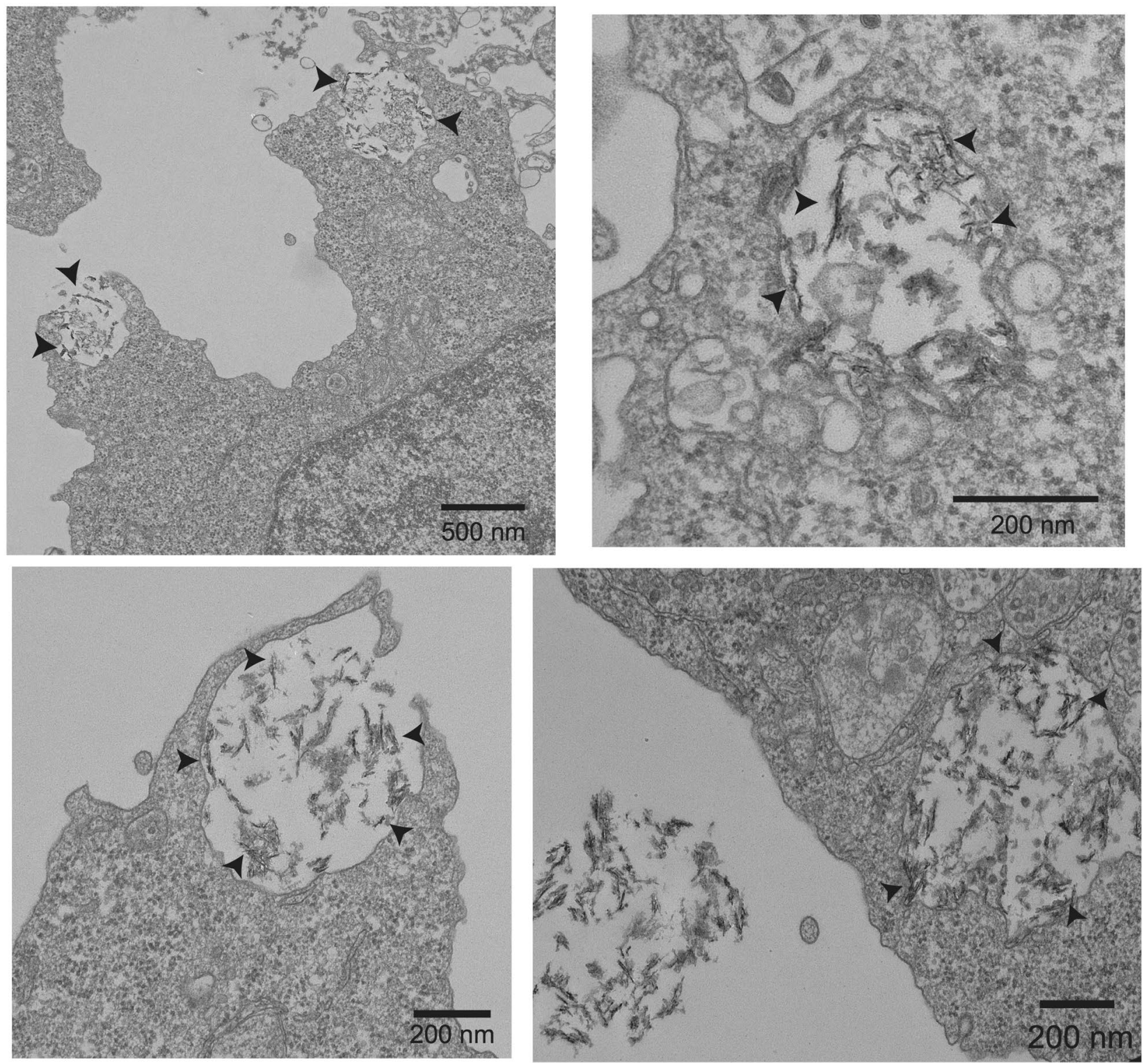

Extended Data Fig. 3 | Alum particles are internalized by B cells in vitro. (a) TEM images of sections of Ramos B cells in the absence of alum.

Representative images are shown from a total of 20 cells imaged. (b) gIVRC01-expressing Ramos B cells ( 2 million/mL) were incubated with pSer ${ }_{8}-e O D$ $(1 \mathrm{ug} / \mathrm{mL}$ ) and alum (10 ug/mL) for 1 hour prior to fixation. Arrowheads point to electron dense alum nanofiber clusters. Representative images are shown from a total of 25 cells imaged. 


\section{ARTICLES}

a

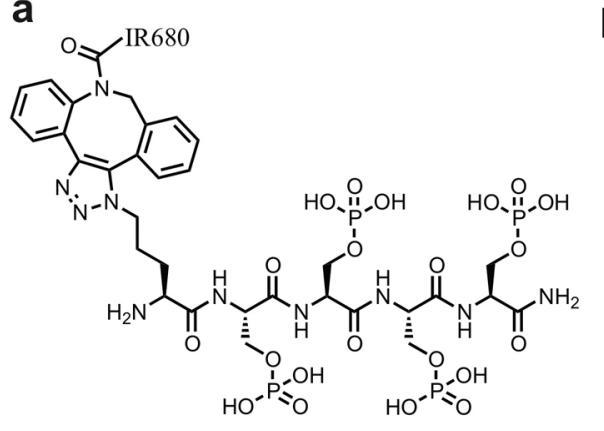

b

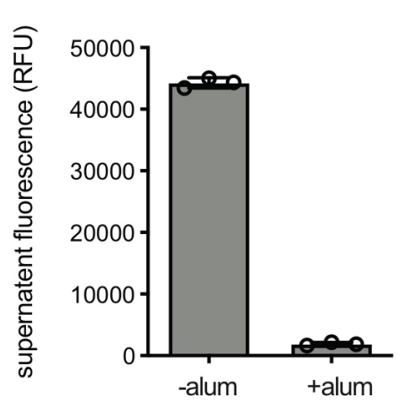

C

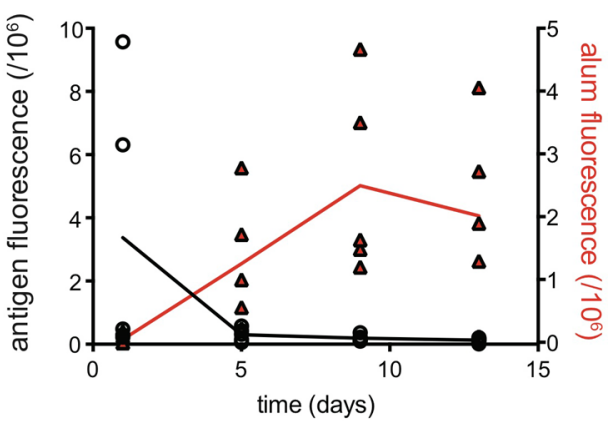

d

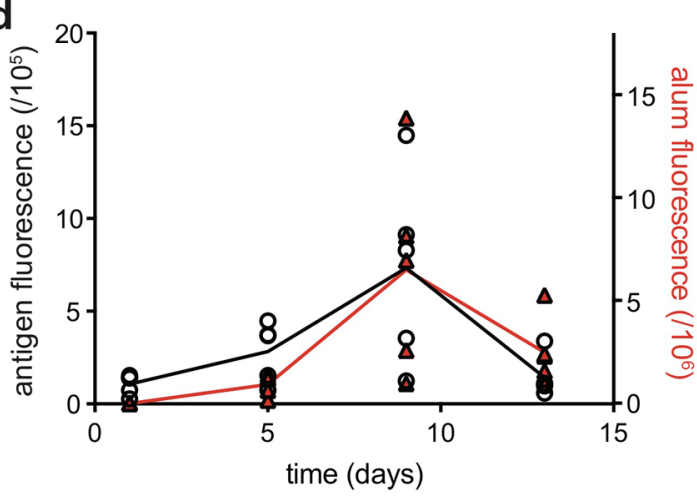

f

subcapsular sinus MФ

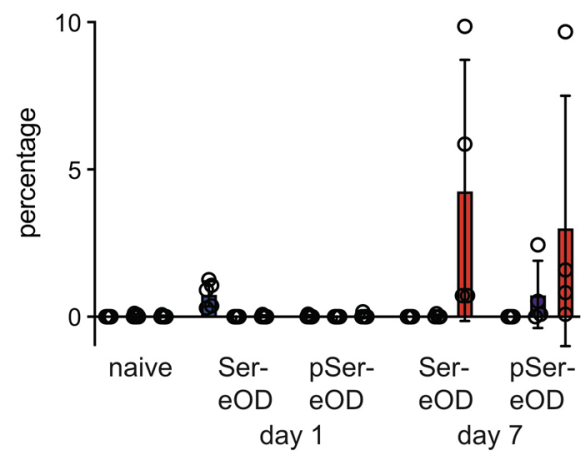

h

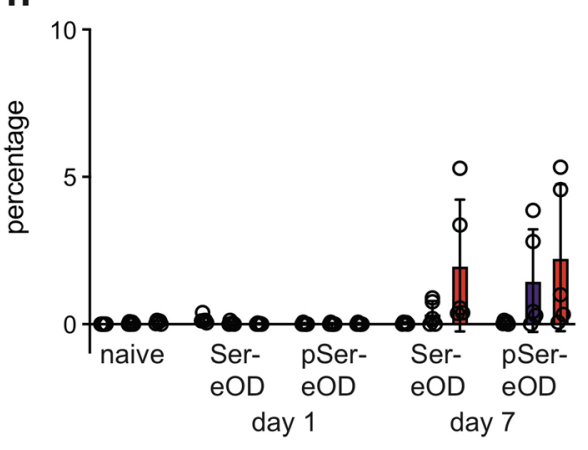

e

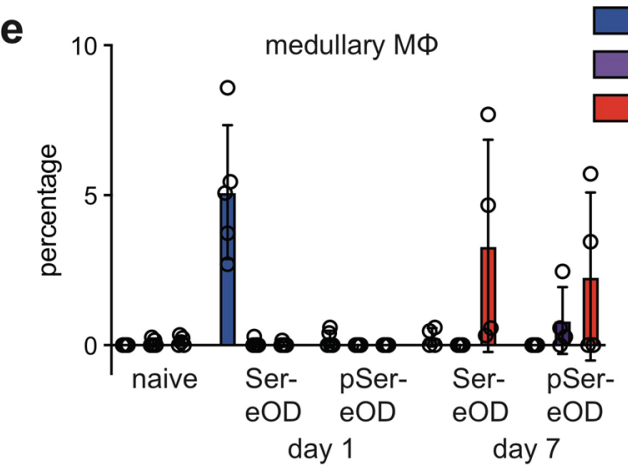

g
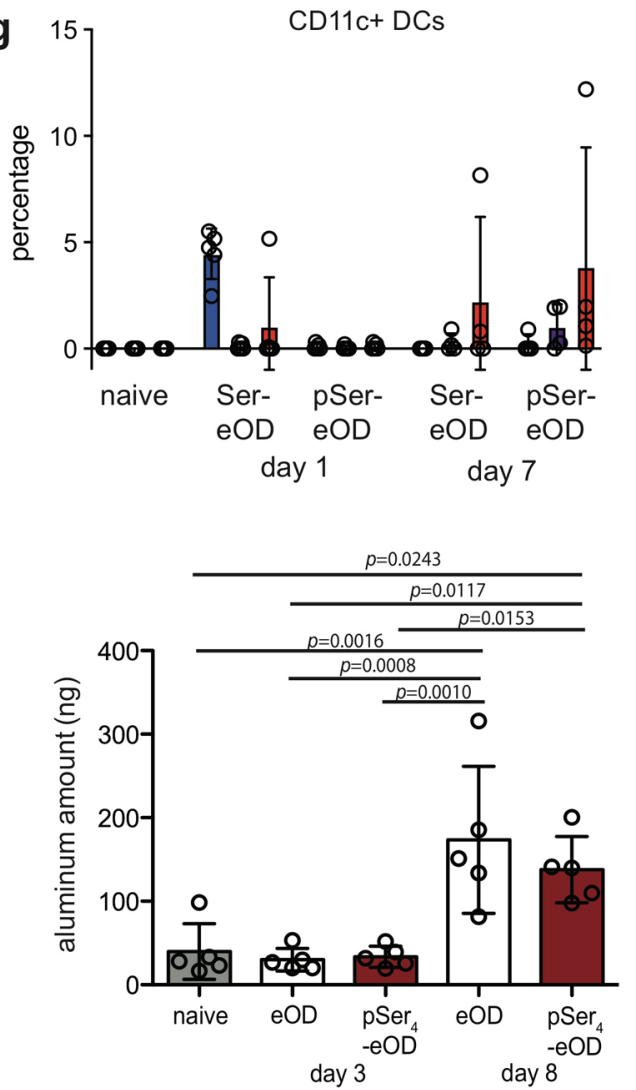

Extended Data Fig. 4 | See next page for caption. 


\section{NATURE MEDICINE}

Extended Data Fig. 4 | Alum particles traffic to lymph nodes and are internalized by antigen presenting cells in vivo. (a) Structure of IR680-pSer ${ }_{4}$

conjugate, synthesized by $\mathrm{Cu}$-free click chemistry to directly label alum. (b) pSer-dye labeling of alum is stable even following incubation in serum. IR680$\mathrm{pSer}_{4}$ conjugate was incubated either alone or with alum for 30 minutes, and then $10 \%$ mouse serum was added, and the solution was incubated at $37^{\circ} \mathrm{C}$ for 72 hours. Data represents the fluorescence measurements of the supernatant after centrifugation to remove any dye remaining bound to alum. Other dyes (Cy3-DBCO and AlexaFluor488-DBCO) were conjugated in the same manner. ( $n=3$ samples/group) Center lines and error bars represent mean and standard deviation, respectively. (c, d) Groups of BALB/c mice ( $n=5 /$ group) were immunized with $5 \mu$ IR800 dye-labeled Ser ${ }_{4}-\mathrm{eOD}$ (c) or pSer ${ }_{8}$-eOD (d), each mixed with $50 \mu \mathrm{g}$ IR680-labeled alum, and total fluorescence from dLNs was measured in excised tissues at serial time points. Center lines represent mean. (e-h) BALB/c mice ( $n=5$ mice per group for naïve and day 3 groups, $n=4$ mice per group for day 7 groups) were immunized with $p S e r_{8}-e O D$ :alum or $\mathrm{Ser}_{4}$-eOD:alum by s.c. injection, and flow cytometry was performed on draining lymph nodes after 1 or 7 days. Alum was labeled with pSer ${ }_{4}$-AF488, and eOD was labeled with AF647. Center lines and error bars represent mean and standard deviation, respectively. (i) ICP-MS measurements of aluminum in inguinal LNs of naïve mice and mice that were immunized s.c. 3 or 8 days prior to measurement. ( $n=5$ mice/group). Center lines and error bars represent mean and standard deviation, respectively. 
a

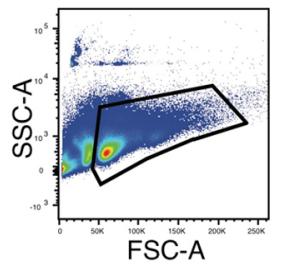

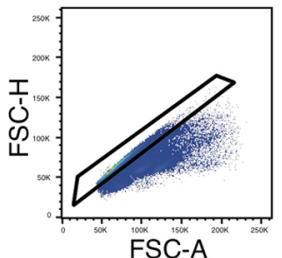

FSC-A

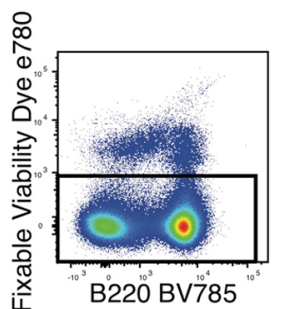

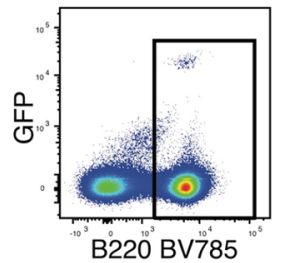
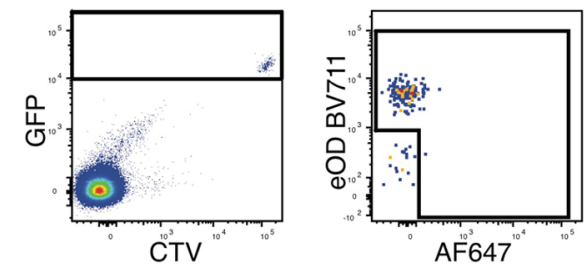

C

b
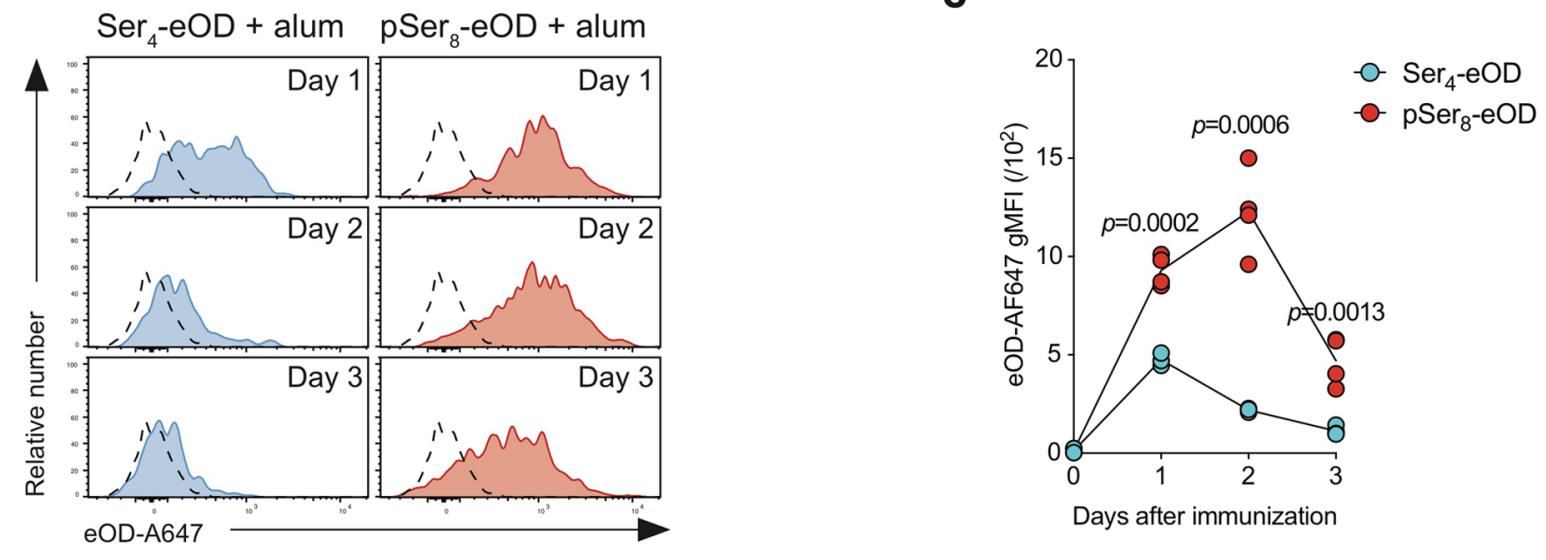

d

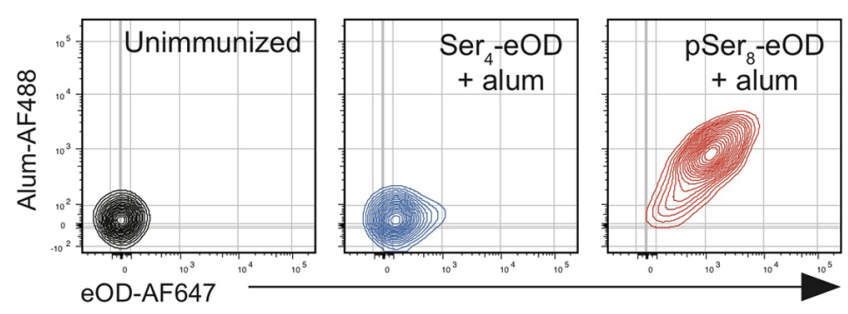

e

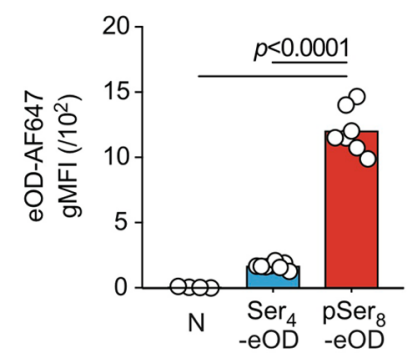

$\mathbf{f}$

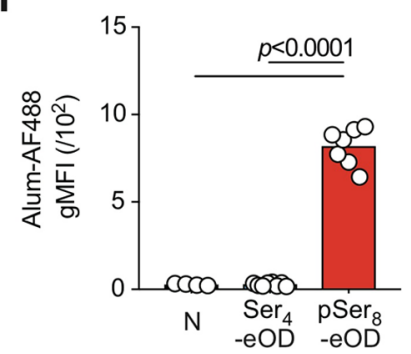

Extended Data Fig. 5 | VRC019HL cells take up eOD bound to alum particles in vivo. (a) Gating strategy used to identify GFP+ VRCO18HL B cells. In vivoacquired eOD antigen had been labeled with AF647 prior to injection. B cells were additionally stained ex vivo with BV711-labeled eOD to further confirm

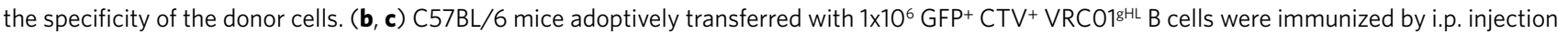
of $5 \mu$ g AF647-labelled Ser ${ }_{4}$-eOD-GT8 or pSer 8 -eOD-GT8 together with $1 \mathrm{mg}$ alum. (b) Representative flow cytometry analysis of VRC018HL B cells. (c) Quantitation of antigen uptake over time. Lines indicate the mean. Data are representative of two independent experiments, $n=3$ mice for d1/2 Ser ${ }_{4}^{-}$ eOD-GT8, $n=4$ mice for d3 Ser ${ }_{4}$-eOD-GT8 and d1-3 pSer ${ }_{8}$-eOD-GT8. Statistical analysis was performed using Two-tailed Student $t$-test. (d-f) C57BL/6 mice adoptively transferred with $1 \times 10^{6} \mathrm{CTV}^{+} \mathrm{VRC018 \textrm {HL }}$ B cells were immunized by i.p. injection of $5 \mu \mathrm{g}$ AF647-labelled Ser ${ }_{4}$-eOD-GT8 or pSer ${ }_{8}$-eOD-GT8 together with $1 \mathrm{mg}$ pSer-AF488 labelled alum. (d) Representative flow cytometry plot of VRCO ${ }^{\mathrm{gHL}}$ cells. (e) Quantification of eOD-GT8 uptake by VRC018HL cells. ( $f$ ) Quantification of alum uptake by VRCOgHL cells. Bars represent the mean. Data combined from two independent experiments, $n=4$ mice for unimmunized, $n=8$ mice for Ser $_{4}$-eOD-GT8 + alum, $n=7$ mice for pSer $_{8}$-eOD-GT8 + alum. Statistical analysis was performed using One-way ANOVA with Tukey's post-test. 
a
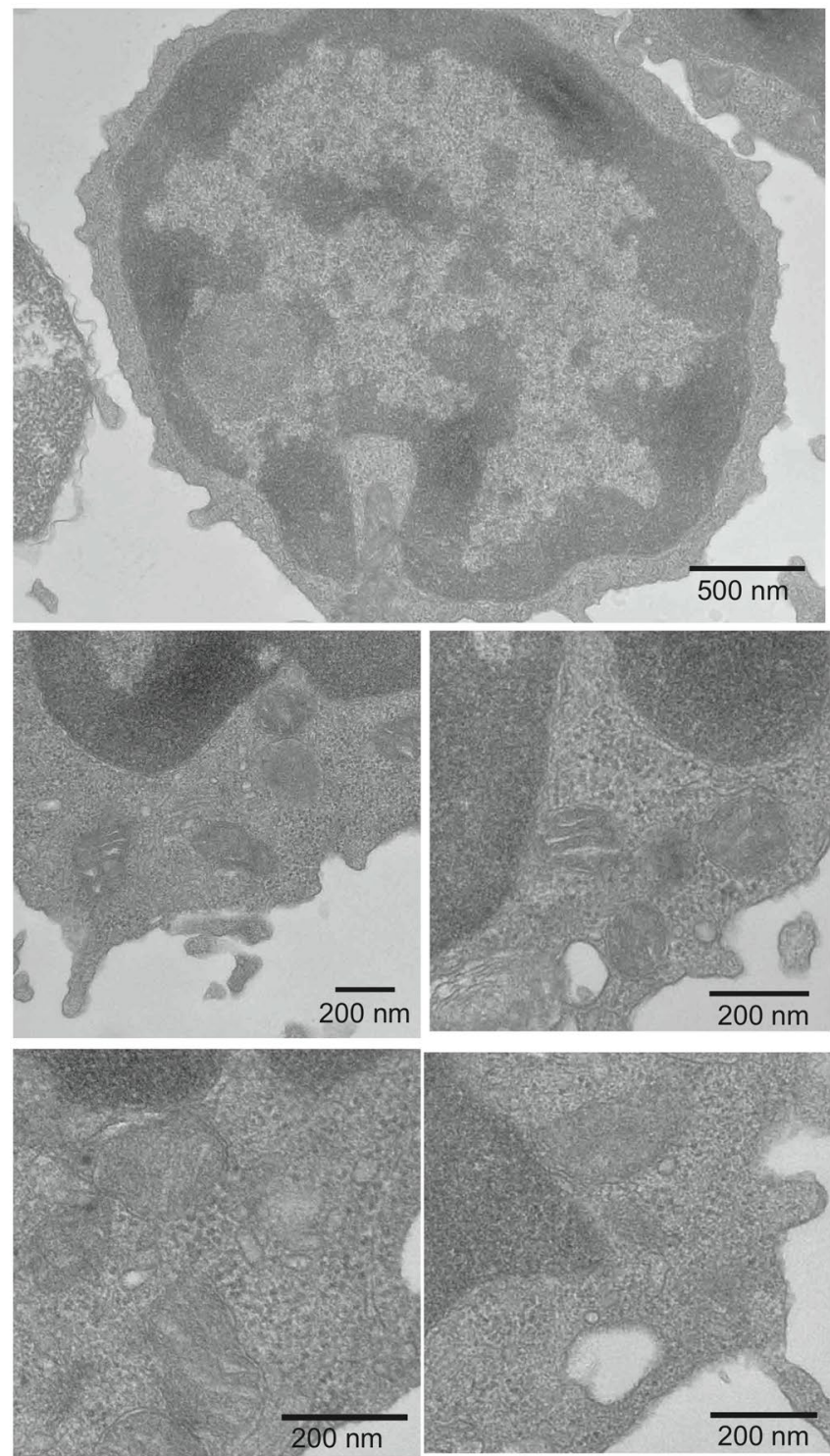

C

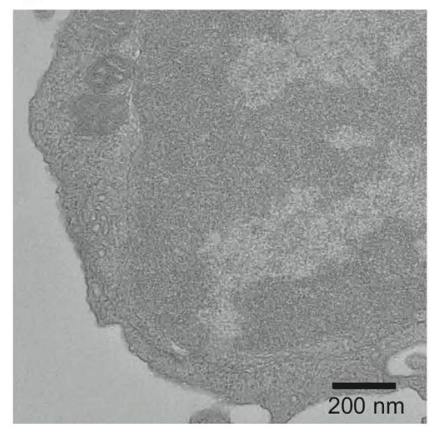

d

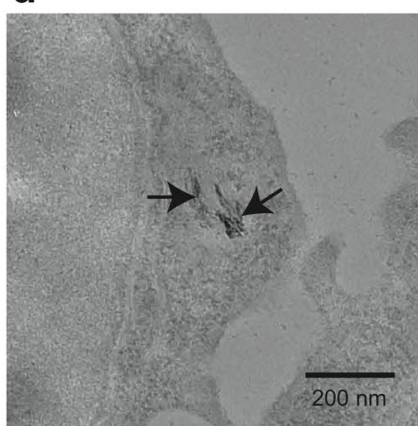

b
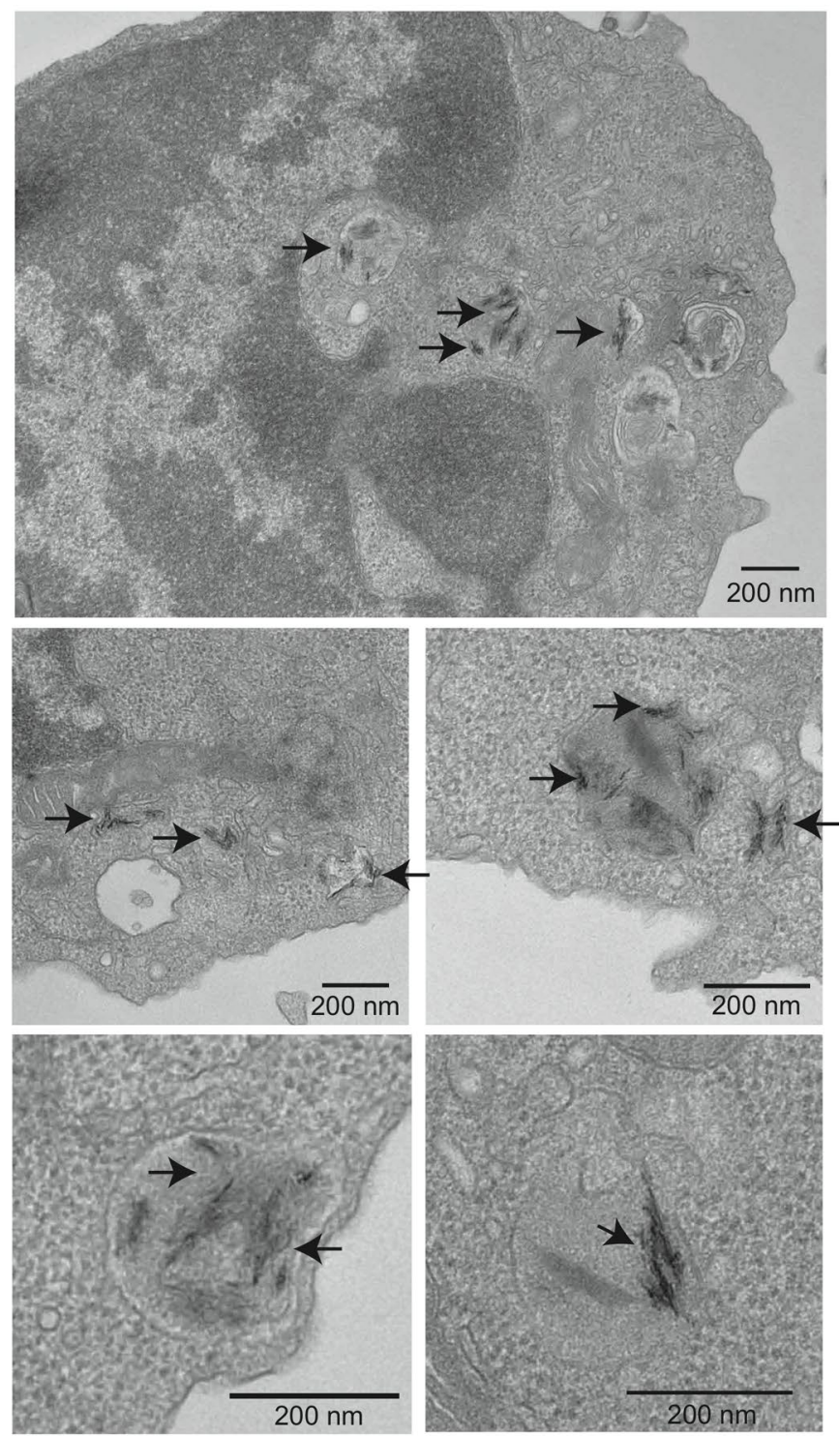

e

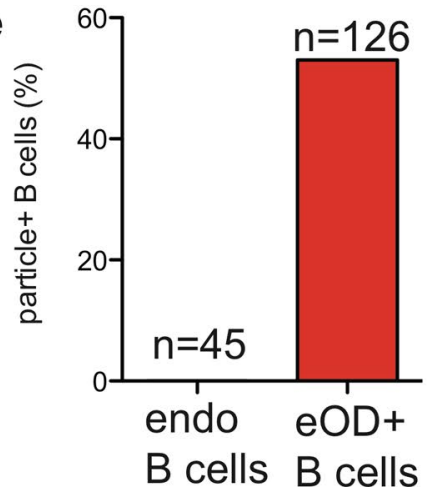

Extended Data Fig. 6 | TEM of sorted B cells after immunization with eOD-GT5 or eOD-GT8. (a, b) Mice were adoptively transferred with VRC018HL B cells then immunized with fluorescent $\mathrm{pSer}{ }_{8}-\mathrm{eOD}-\mathrm{GT} 5$ and alum i.p. Two days after immunization, endogenous (a) and eOD-GT5+ VRC018HL B cells (b) were sorted by flow cytometry, fixed, and sectioned for TEM imaging. 145 (a) and 153 (b) cells were analyzed, and representative images are shown.

(c, d) Identical experimental conditions were used, with pSer ${ }_{8}-\mathrm{eOD}-\mathrm{GT} 8$ as the antigen, for TEM imaging of endogenous (c) and eOD-GT8 ${ }^{+}$VRCO18HL B cells (d). 126 cells were imaged for (d) and 45 cells were imaged for (c). (e) Quantification of images from (c) and (d). Arrowheads point to electron dense alum clusters. 
a

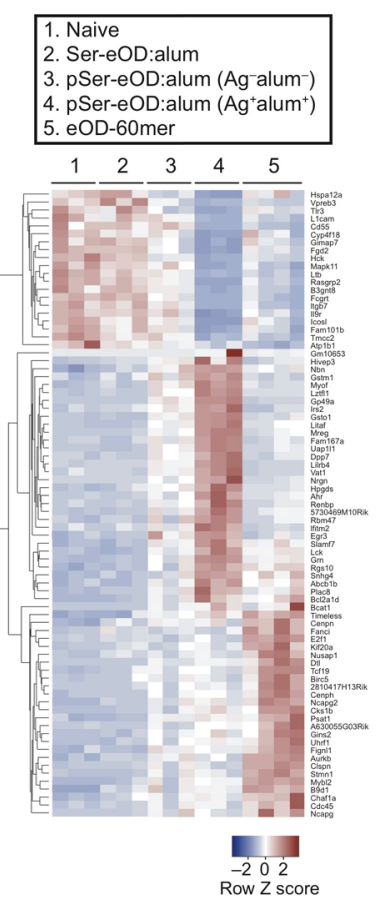

b

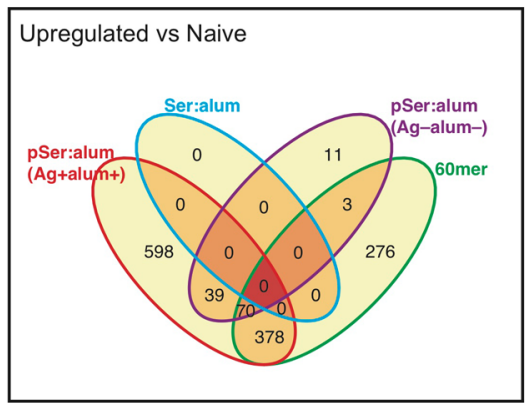

Downregulated vs Naive

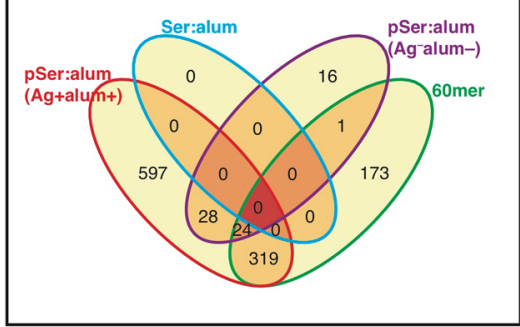

C
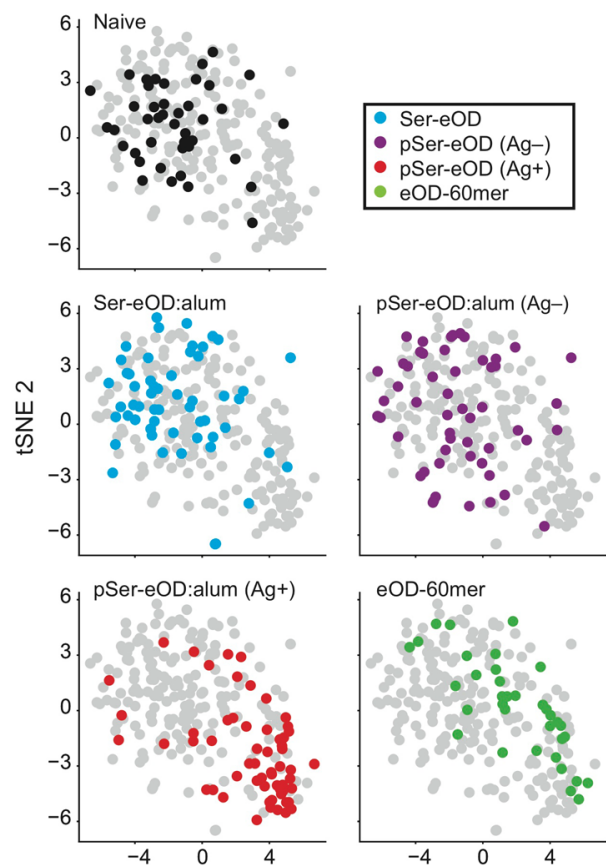

eOD-60mer

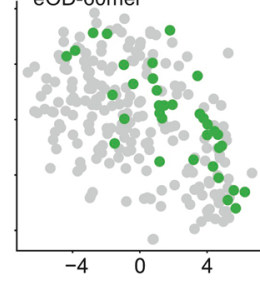

tSNE 1
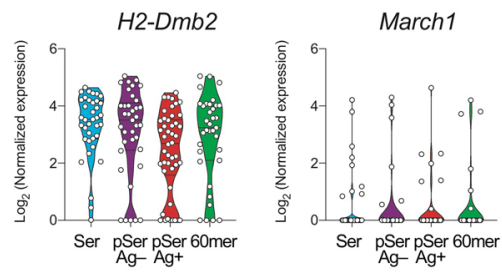

f

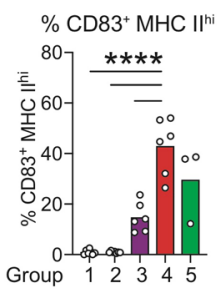

g
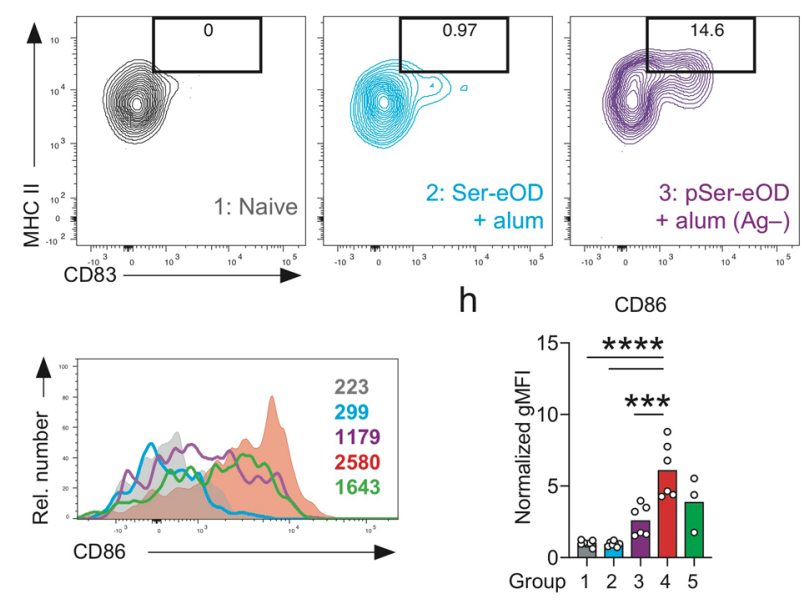
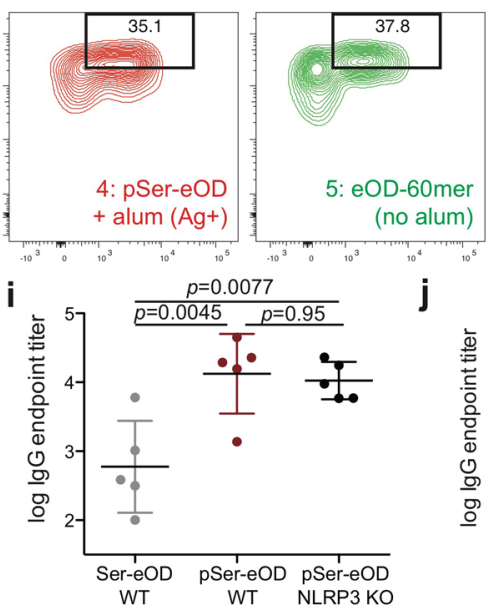

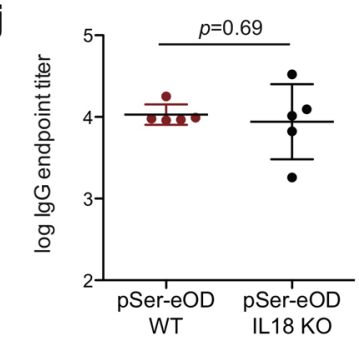

Extended Data Fig. 7 | See next page for caption. 
Extended Data Fig. 7 | RNA-seq analysis of VRC01-class bnAb precursors. a) Bulk RNA-seq analysis showing the row-wise $z$ score of differentially expressed genes in VRC019HL B cells from vaccinated mice compared to naive cell (2-fold cutoff, adj $p<0.01$ ). $n=3$ mice for group 1-4, $n=4$ mice for group 5. (b) Venn diagrams summarizing bulk RNA-seq analysis showing the number of differentially expressed genes in VRC018HL cells from each group compared to naive cells (1.5-fold cut-off, adj $p<0.05$, DeSeq2 pairwise comparisons). (c) $t$-Distributed stochastic neighboring embedding (tSNE) twodimensional plot of single-cell RNA-seq data shows different clustering patterns of VRC018 ${ }^{\mathrm{HL}}$ cells from different immunization groups. $n=42$ cells ( 4 mice) for naïve, $n=55$ cells ( 4 mice) for Ser4-eOD, $n=53$ cells ( 4 mice) for pSer-eOD (Ag-), $n=58$ cells for pSer-eOD (Ag+) ( 4 mice), $n=35$ cells (2 mice) for 60 mer. Pooled data from two adoptive transfer experiments. (d) Normalized expression of MHC II genes and March1 by VRCO1 ${ }^{\mathrm{HL}} \mathrm{cells}$. Horizontal lines in violin plots indicate the first quartile, the median, and the third quartile. Each circle represents a cell. $n=37$ cells ( 2 mice) for SereOD + alum, $n=37$ cells ( 2 mice) for pSer-eOD + alum (Ag-), $n=46$ cells ( 2 mice) for pSer-eOD + alum (Ag+), $n=35$ cells ( 2 mice) for eOD-60mer. (e-h) C57BL/6 mice adoptively transferred with $1 \times 10^{6} \mathrm{CTV}^{+} \mathrm{VRC018 \textrm {HL }}$ B cells were immunized by i.p. injection of 10 $\mu \mathrm{g}$ AF647-labelled Ser ${ }_{4}$-eOD-GT5 or pSer ${ }_{8}$-eOD-GT5 together with $1 \mathrm{mg}$ alum. (e) Flow cytometry analysis showing changes in MHC II and CD83 expression patterns on VRC018HL B cells. Number indicates percentage of $\mathrm{CD} 83^{+} \mathrm{MHC} \mathrm{II}{ }^{\mathrm{hi}}$ VRC018HL $\mathrm{B}$ cells. Two independent experiments were performed with similar results. (f) Bar graphs show percentage of CD83+ $\mathrm{MHC} \mathrm{II}{ }^{\text {hi }}$ VRC018HL B cells in (E). Bars represent the mean. Pooled data from two experiments. $n=7$ for group 1 and $2, n=6$ for group 3 and 4, n=3 for group 5. (g) Flow cytometry analysis showing changes in CD86 expression patterns on VRC01gHL B cells. Two independent experiments were performed with similar results. (h) Bar graphs show gMFI of CD86 on VRC018HL cells in (G) normalized to unprimed group. Bars represent the mean. Data combined from two independent experiments. ${ }^{\star \star \star}, p=0.002 ;{ }^{\star \star \star \star}, p<0.0001$ by One-way ANOVA with Tukey's post-test. (i) Day 28 sera from C57BL/6

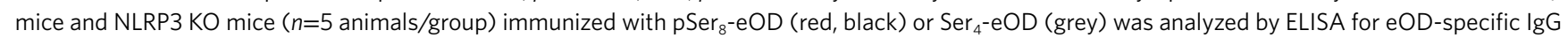
antibody responses. Statistical comparison by One-way ANOVA with Tukey's post-test. (j) Day 14 sera from BL/6 mice (red) and IL18 KO mice (black) ( $n=5$ animals/group) immunized with pSer ${ }_{8}$-eOD was analyzed for eOD-specific lgG antibody responses. $p=0.69$ by two-tailed Student's t-test. 
a
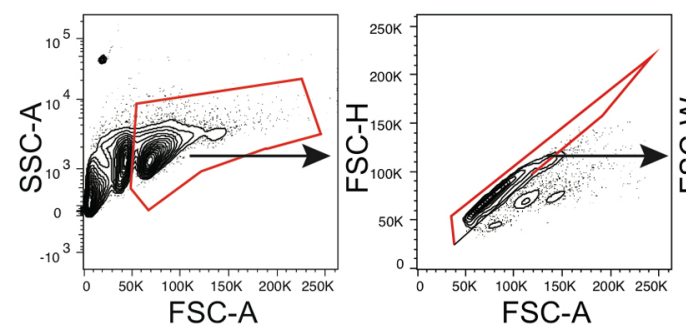

FSC-A

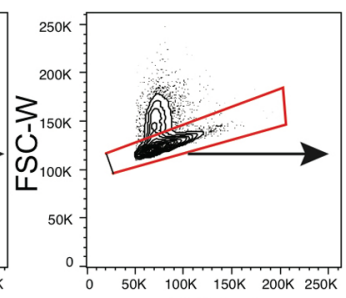

FSC-A
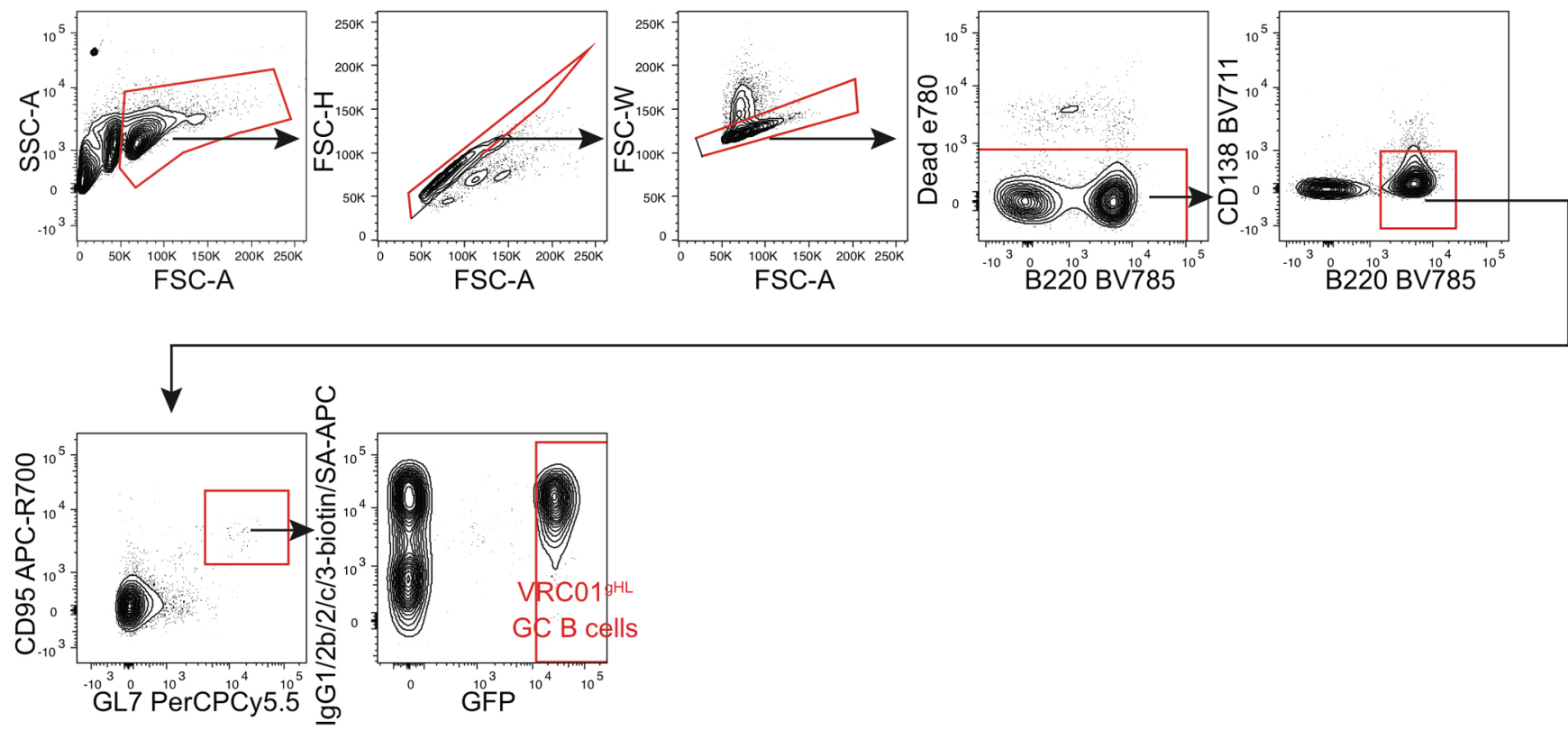

b
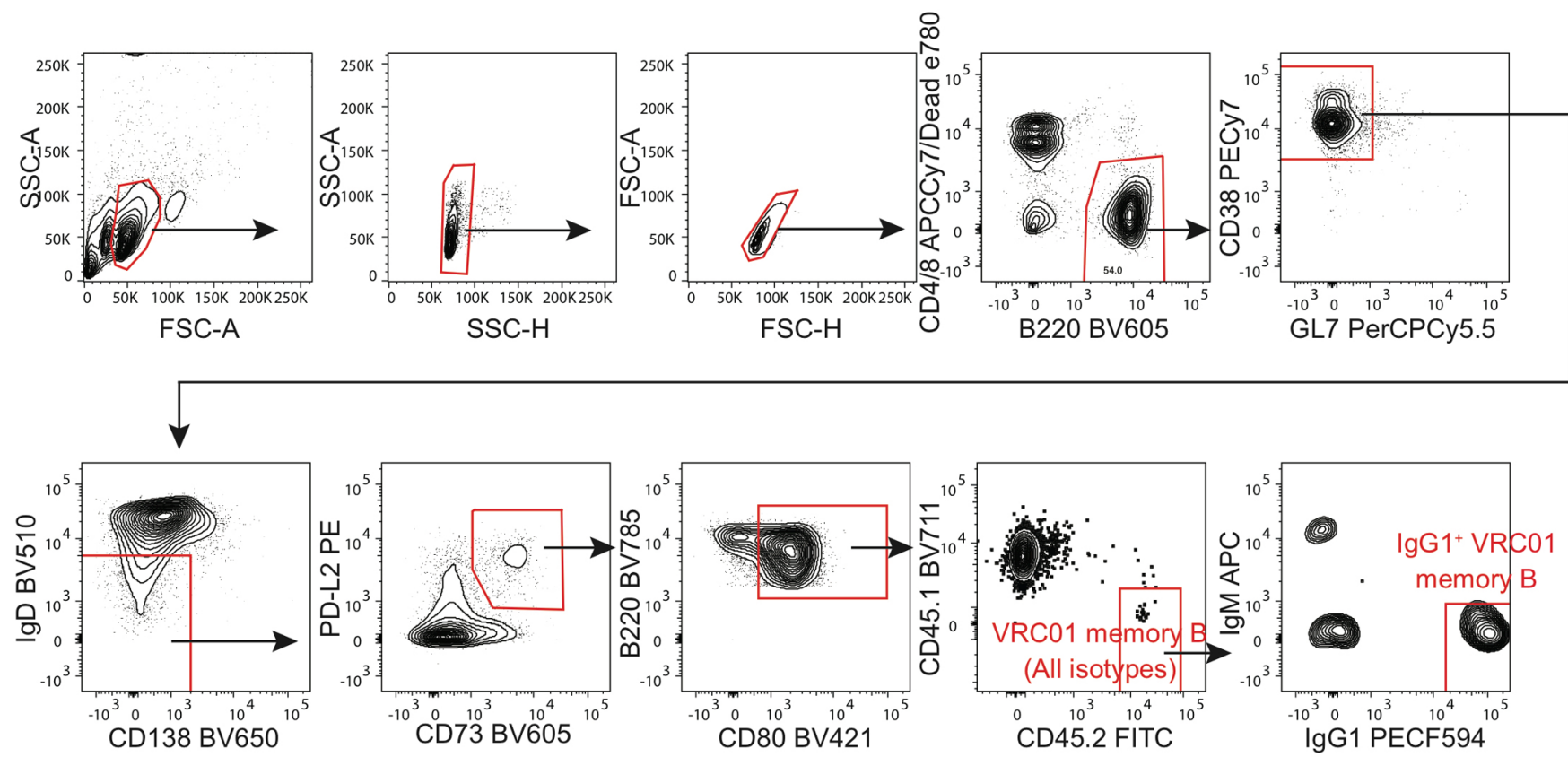

Extended Data Fig. 8 | Flow cytometry gating for VRC01 ${ }^{\mathrm{HL}}$ B cell populations. (a) Gating strategy for VRC018HL GC B cells. (b) Gating strategy for GCderived $\mathrm{VRCO}{ }^{\mathrm{HH}}$ memory $\mathrm{B}$ cells. 

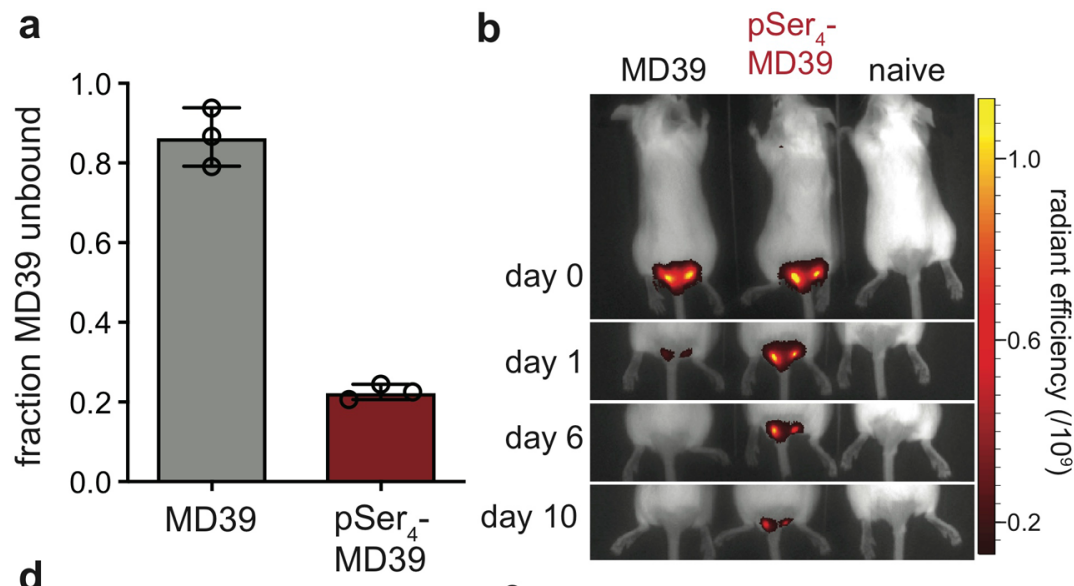

C
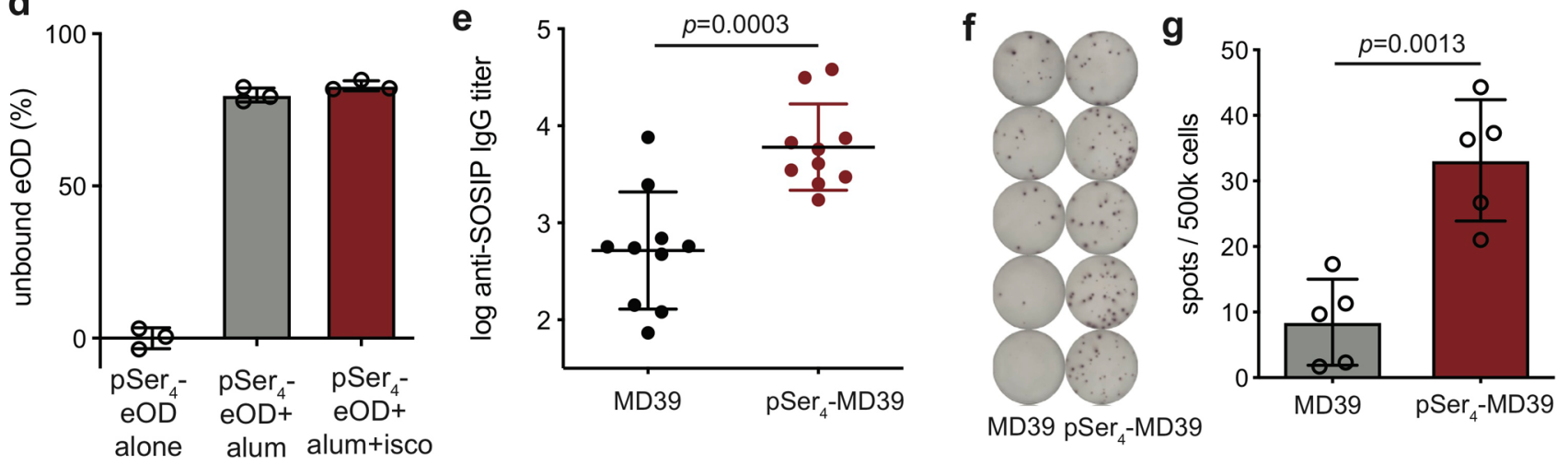

h
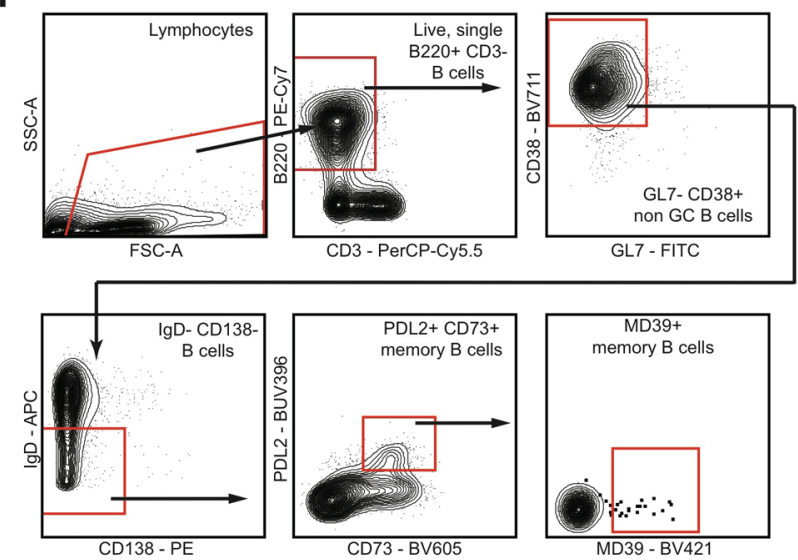

k

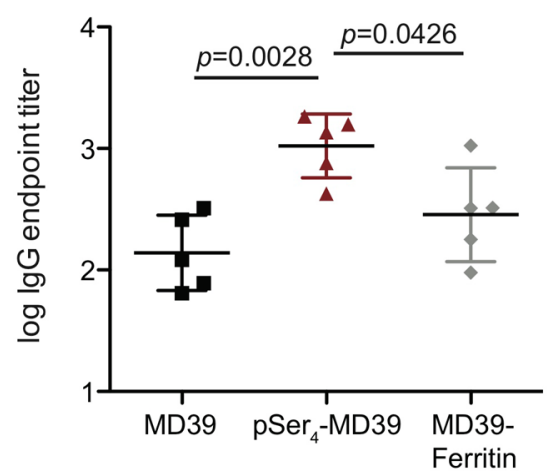

i

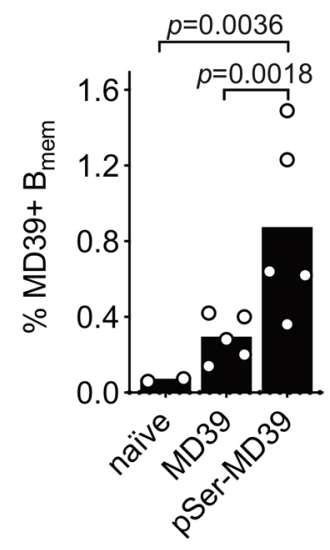

j

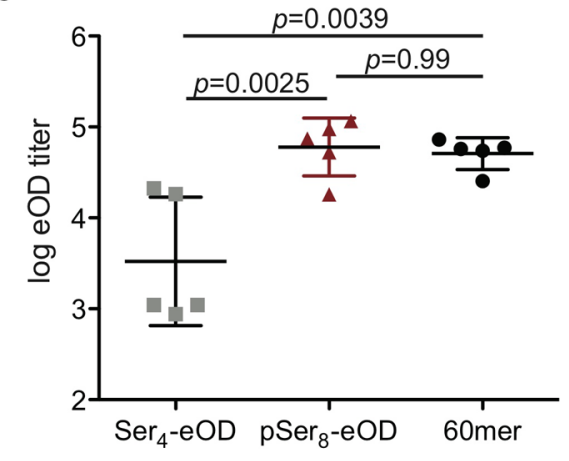

Extended Data Fig. 9 | See next page for caption.

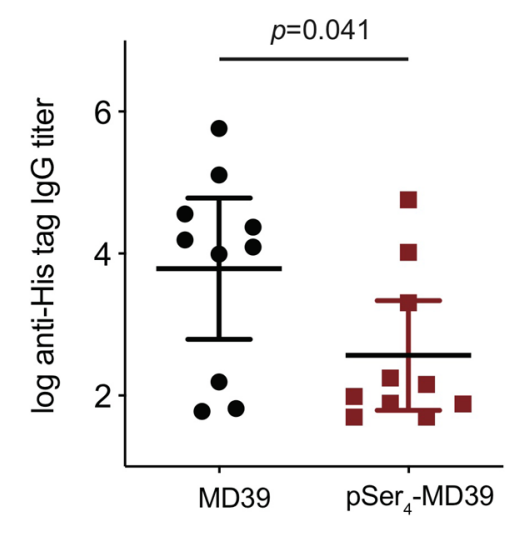

m

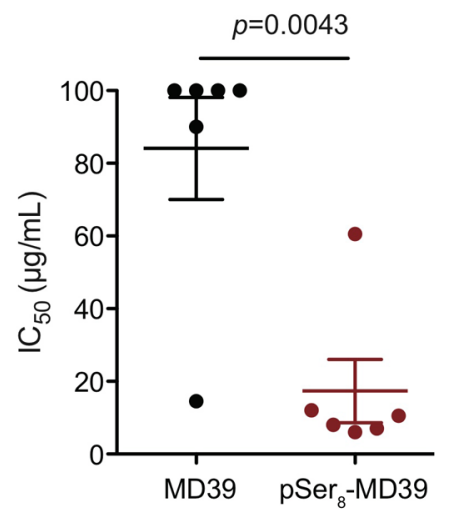


Extended Data Fig. 9 | pSer-MD39 binds to alum, enhancing humoral and neutralization responses. (a) After incubation of MD39 trimer with alum for 30 minutes, 10\% mouse serum was added, and solution was incubated for 24 hours. Binding of MD39 to alum was determined by the presence of MD39 in the supernatant after pelleting alum by centrifugation. MD39 concentration was measured by ELISA relative to a standard curve. ( $n=3$ samples/group).

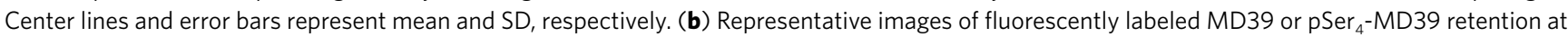
the injection site after immunization with alum in BALB/c mice $(n=4$ animals/group). (c) Quantification of IVIS images from (b). Data represents mean \pm SD. (d) ISCOM-like saponin adjuvant does not inhibit pSer-immunogen binding to alum. Fluorescently labeled pSer ${ }_{4}-\mathrm{eOD}(10 \mathrm{ug} / \mathrm{mL}) \mathrm{was} \mathrm{combined}$ with either alum or alum and saponin nanoparticles (labeled isco). After incubation for 24 hours in the presence of $10 \%$ mouse serum, the percentage of unbound eOD was measured by fluorescence. The fluorescence signal of $\mathrm{pSer}_{4}$-eOD in the absence of alum was normalized to $100 \%$ ( $n=3$ samples/ group). Center lines and error bars refer to mean and SD, respectively. (e) BALB/c mice ( $n=10 /$ group, pooled from two immunizations) were immunized with $5 \mu \mathrm{g}$ MD39 or pSer ${ }_{4}-\mathrm{MD} 39$ mixed with $50 \mu \mathrm{g}$ alum and $5 \mu \mathrm{g}$ saponin adjuvant. IgG titers from individual mice at week 6 . . Statistical analysis by two-tailed Student's $t$-test of the log-transformed data. Data are represented as mean \pm SD of the log-transformed data. (f) Representative images of bone marrow ELISPOT plates for antibody-secreting cells at 3 months post immunization using the same conditions describe in (e). Experiments were performed twice, after two separate immunizations ( $n=5$ mice/group for each experiment). (g) Quantification of mean bone marrow MD39 trimerspecific ELISPOT responses described in ( $f$ ). Statistical analysis by two-tailed Student's $t$-test ( $n=5$ animals/group). Center lines and error bars refer to mean and SD, respectively. (h) Flow cytometry gating for identification of Env trimer-specific memory B cells. (i) Groups of BALB/c mice ( $n=5$ mice/

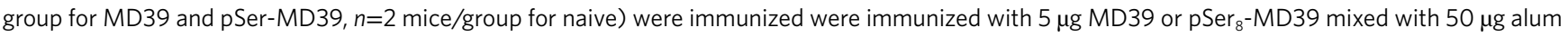

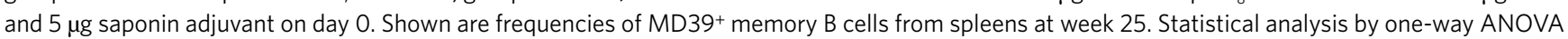
with Tukey's multiple comparison test. Center lines represent mean. (j, k) BALB/c mice ( $n=5$ mice/group) were immunized with 5 ug antigen, and titers were measured four weeks after s.c. immunization. (j) Ser ${ }_{4}-\mathrm{eOD}$, alum-binding pSer ${ }_{8}-\mathrm{eOD}$, and eOD 60mer were used as antigens in combination with alum. Statistical analysis was performed using one-way ANOVA with Tukey's post-test ( $n=5$ mice/group) of the log-transformed data. Center lines and error bars refer to mean and SD, respectively, of the log-transformed data. (k) MD39 control, pSer ${ }_{4}-\mathrm{MD} 39$, and MD39-ferritin were used as antigens in combination with alum. Statistical analysis was performed using one-way ANOVA with Tukey's post-test ( $n=5$ mice $/$ group) of the log-transformed data. Center lines and error bars refer to mean and SD, respectively, of the log-transformed data. (I) BALB/c mice ( $n=10$ mice/group) were immunized with $5 \mu \mathrm{g}$ MD39 or pSer ${ }_{4}-M D 39$ mixed with $50 \mu$ g alum on day 0 with ISCOM-like adjuvant. His tag-specific IgG titers at day 63 . Statistical analysis by two-tailed Student's $t$-test of the log-transformed data. Center lines and error bars refer to mean and SD, respectively, of the log-transformed data. (m) Rabbits were immunized with MD39:alum or pSer ${ }_{8}$ MD39:alum on weeks 0 and 8 ( $n=6$ rabbits/group). Purified IgG antibodies from rabbit sera collected on week 10 was measured for neutralization against autologous tier 2 virus. Statistical analysis by Mann-Whitney test. Center lines and error bars refer to mean and $\mathrm{SD}$, respectively. 
a

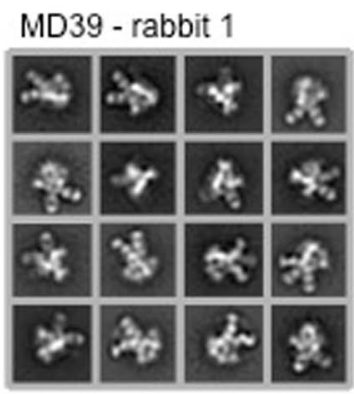

MD39 - rabbit 2
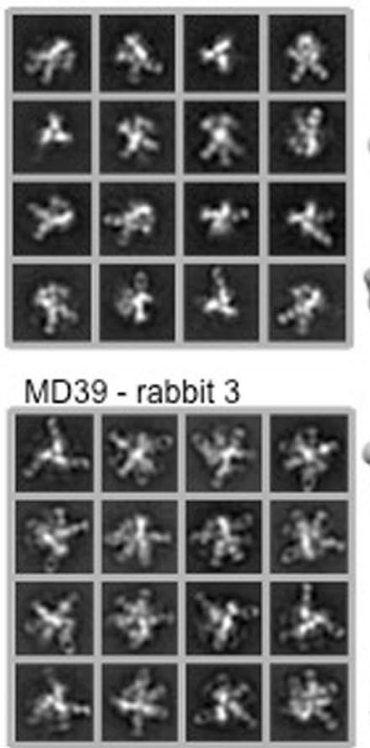
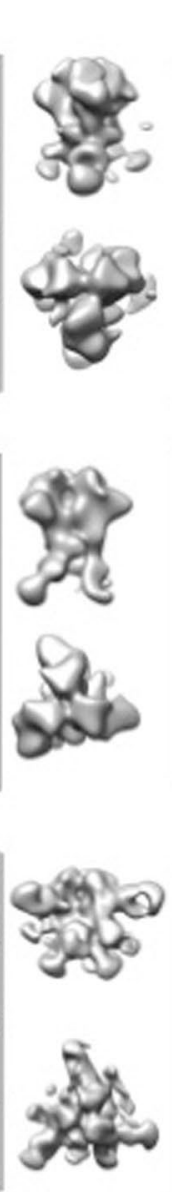

b
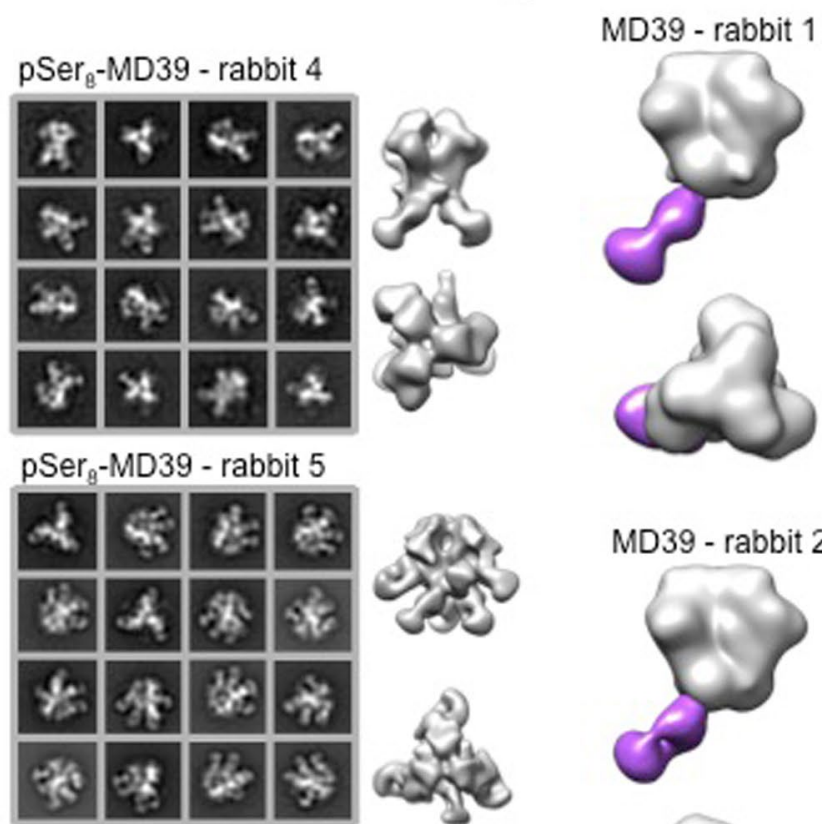

pSer - MD39 - rabbit 4
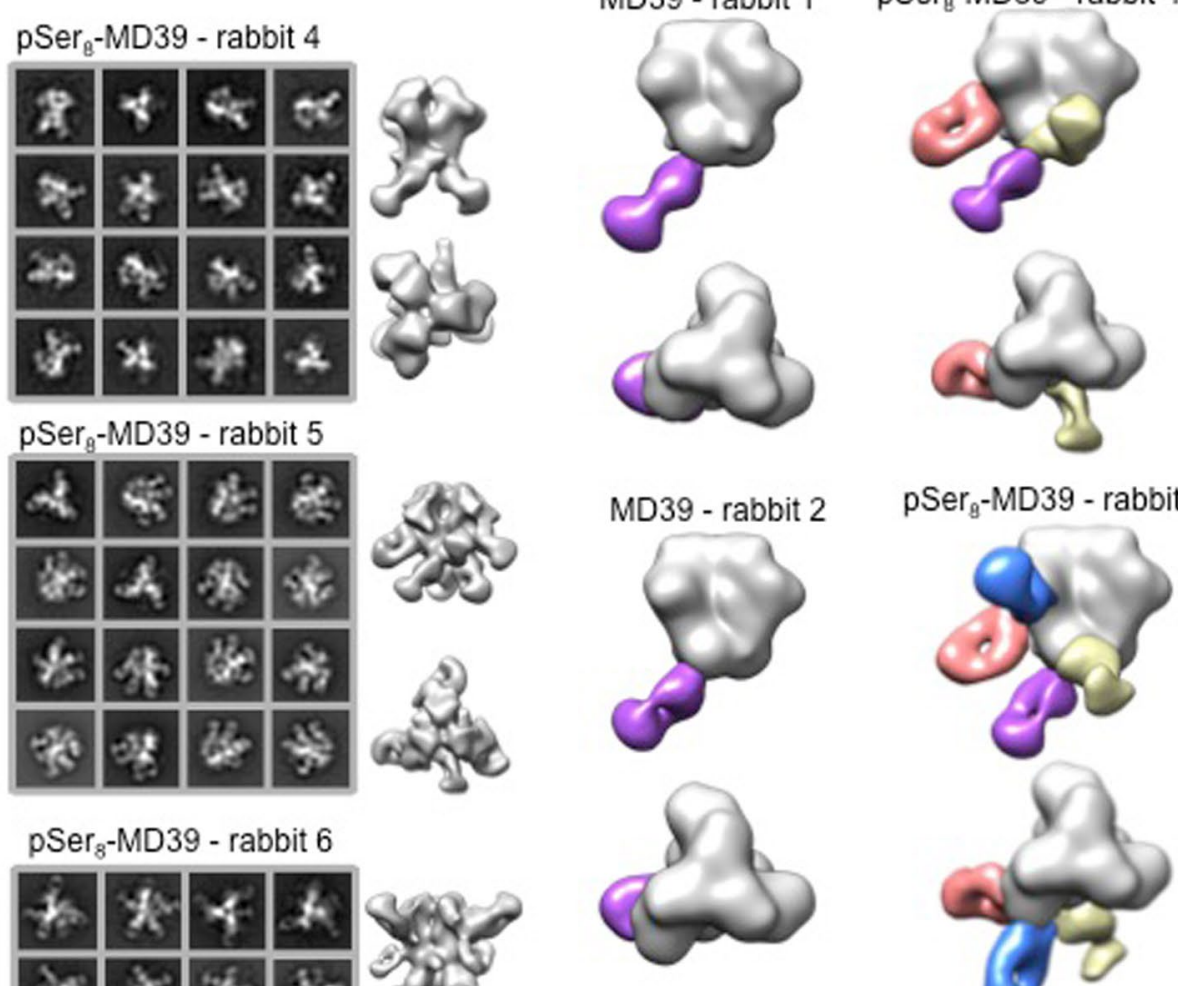

pSer - -MD39 - rabbit 5
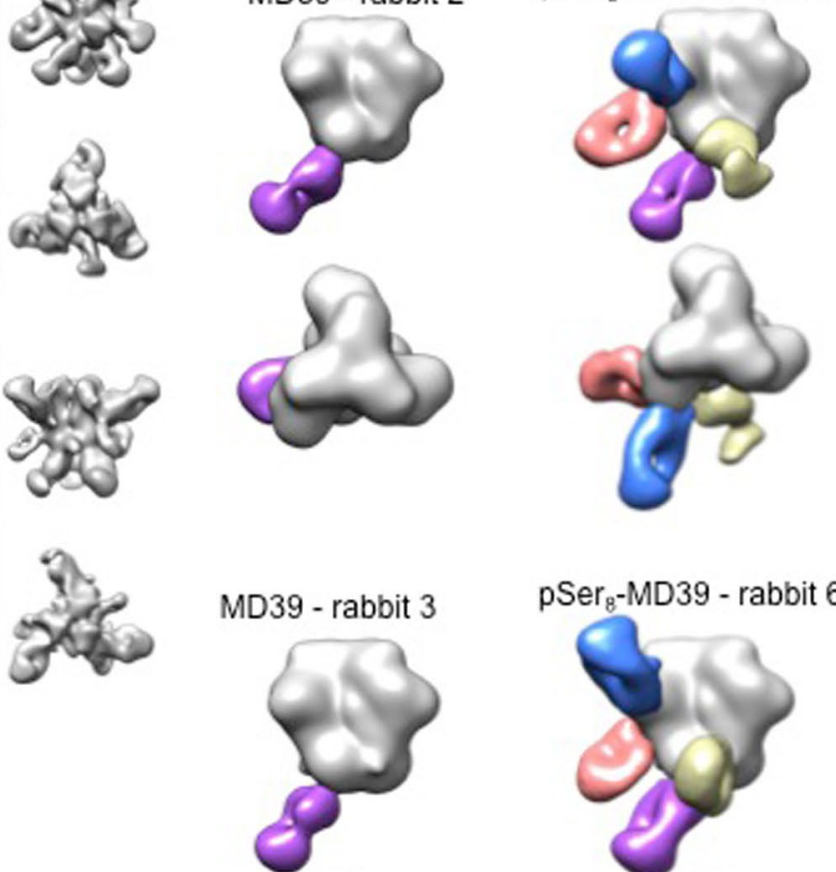
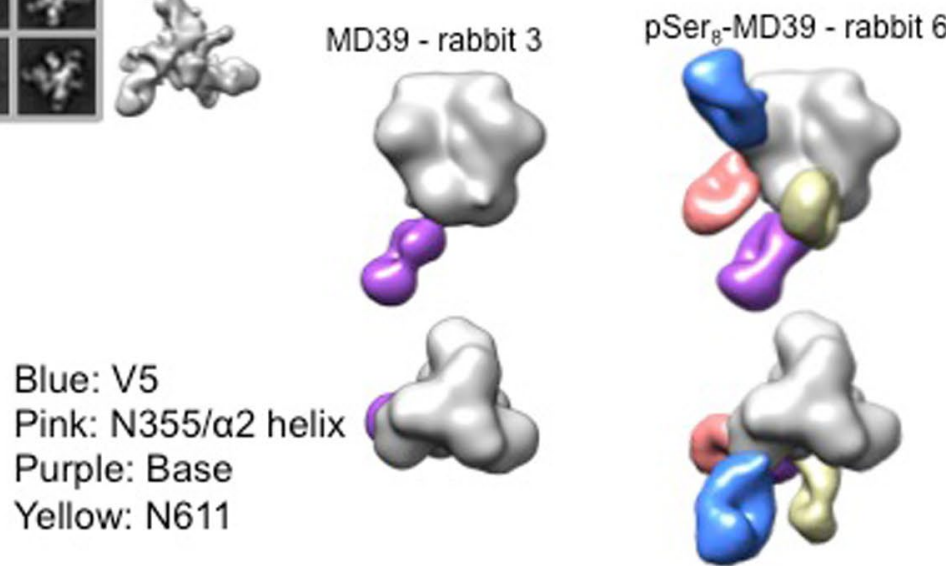

Extended Data Fig. 10 | EM averages and reconstructions. (a) Representative subset of single particle nsEM 2D class averages (left) and corresponding reconstructed 3D maps (right). The 3D reconstructions are an average composite of most particles in each dataset and reveal the epitopes targeted in each serum sample as labeled. (b) Per animal epitope analysis showing a single segmented Fab per epitope as determined by further 3D classification of average composite reconstructions shown in Supplementary Figure 7. Colors represent the region of trimer where Fabs bind (blue - V5, pink - N355/alpha2 helix, purple - base, and yellow - N611). Three separate rabbit sera were analyzed for both MD39 and pSer 8 -MD39 immunizations. 


\section{Reporting Summary}

Nature Research wishes to improve the reproducibility of the work that we publish. This form provides structure for consistency and transparency in reporting. For further information on Nature Research policies, see Authors \& Referees and the Editorial Policy Checklist.

\section{Statistics}

For all statistical analyses, confirm that the following items are present in the figure legend, table legend, main text, or Methods section.

n/a Confirmed

$\bigotimes$ The exact sample size $(n)$ for each experimental group/condition, given as a discrete number and unit of measurement

$\square$ A statement on whether measurements were taken from distinct samples or whether the same sample was measured repeatedly

$\square$ The statistical test(s) used AND whether they are one- or two-sided

Only common tests should be described solely by name; describe more complex techniques in the Methods section.

Х $\square$ A description of all covariates tested

$\square$ A description of any assumptions or corrections, such as tests of normality and adjustment for multiple comparisons

$\triangle$ A full description of the statistical parameters including central tendency (e.g. means) or other basic estimates (e.g. regression coefficient)

$\triangle$ AND variation (e.g. standard deviation) or associated estimates of uncertainty (e.g. confidence intervals)

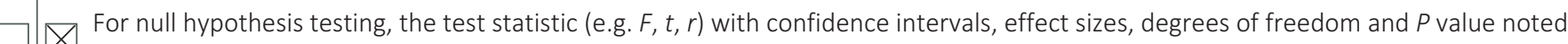

$\triangle$ Give $P$ values as exact values whenever suitable.

Х $\square$ For Bayesian analysis, information on the choice of priors and Markov chain Monte Carlo settings

Х $\square$ For hierarchical and complex designs, identification of the appropriate level for tests and full reporting of outcomes

$\triangle \square$ Estimates of effect sizes (e.g. Cohen's d, Pearson's $r$ ), indicating how they were calculated

Our web collection on statistics for biologists contains articles on many of the points above.

\section{Software and code}

Policy information about availability of computer code

Data collection

Flow cytometry data were obtained using BD FACSDiva software. ELISA plates and plate-based fluorescence experiments were measured by using Tecan Infinite M200 Pro absorbance/fluorescence plate reader and software. ELISPOT measurements were taken using CTL Immunospot Analyzer and associated software. Fluorescence measurements in lymph nodes were made using a LI-COR Odyssey reader and LI-COR imaging software. Confocal images were taken on an Olympus X71 microscope and used accompanying software.

Data analysis

FlowJo v10.5 was used for flow cytometry analysis. For scRNA-seq, Tophat v1.4.1, HTSeq-count v0.11.0, DESeq2 v3.1, Seurat v2.3.4, RStudio v1.1453, and Squencher v5.1 were used. GraphPad Prism 8 was used for data analysis and plots. Fiji (ImageJ v2.0.0) was used for image processing of confocal images. IVIS images were analyzed using Living Image v4.5.

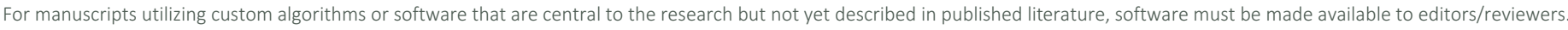
We strongly encourage code deposition in a community repository (e.g. GitHub). See the Nature Research guidelines for submitting code \& software for further information.

\section{Data}

Policy information about availability of data

All manuscripts must include a data availability statement. This statement should provide the following information, where applicable:

- Accession codes, unique identifiers, or web links for publicly available datasets

- A list of figures that have associated raw data

- A description of any restrictions on data availability

All requests for raw and analyzed data and materials are promptly reviewed by the MIT Technology Licensing Office to verify if the request is subject to any intellectual property or confidentiality obligations. Any data and materials that can be shared will be released via a Material Transfer Agreement. 
Please select the one below that is the best fit for your research. If you are not sure, read the appropriate sections before making your selection.
$\bigotimes$ Life sciences
Behavioural \& social sciences
Ecological, evolutionary \& environmental sciences

For a reference copy of the document with all sections, see nature.com/documents/nr-reporting-summary-flat.pdf

\section{Life sciences study design}

All studies must disclose on these points even when the disclosure is negative.

Sample size A sample size of 5 was used to detect a significant difference $(p<0.05)$ between groups with a signal to noise ratio of 2.0 with $80 \%$ power.

Data exclusions In Figure 6g, two data points from the control group were excluded from the area under the curve analysis, since these two mice that had MD39-binding titers that were at background levels in the absence of the base-binding antibody. This exclusion criteria was not preestablished.

Replication $\quad$ All murine experiments report pooled results from multiple experiments or data shown is one representative of at least two experiments. All attempts at replication were successful.

Rabbit studies employed 6 animals/group for biological replicates.

Randomization Randomization was not used for this study. Prior to immunizations, mice were evenly distributed into experimental groups from the same cohort of mice.

Blinding Investigators were blinded for the neutralization analysis of rabbit sera.

\section{Reporting for specific materials, systems and methods}

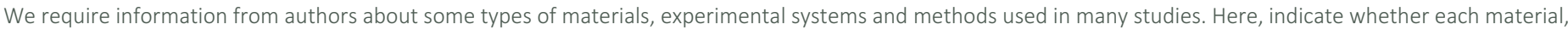

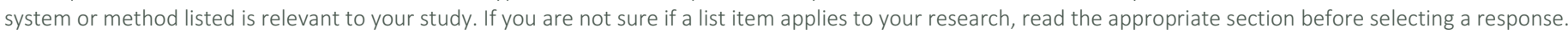

\begin{tabular}{|c|c|c|c|}
\hline \multicolumn{2}{|c|}{ Materials \& experimental systems } & \multicolumn{2}{|c|}{ Methods } \\
\hline $\mathrm{n} / \mathrm{a}$ & Involved in the study & $\mathrm{n} / \mathrm{a}$ & Involved in the study \\
\hline & \ Antibodies & Х & $\square$ ChIP-seq \\
\hline & Eukaryotic cell lines & $\square$ & X Flow cytometry \\
\hline Х & Palaeontology & Х & MRI-based neuroimaging \\
\hline & $\bigotimes$ Animals and other organisms & & \\
\hline Х & Human research participants & & \\
\hline Х & Clinical data & & \\
\hline
\end{tabular}

\section{Antibodies}

Antibodies used

Histology:

anti-mouse B220 AF488 (clone RA3-6B2; Biolegend; 1:100 dilution)

anti-mouse CD35 BV421 (clone 8C12; BD; 1:100 dilution)

Flow Cytometry:

anti-mouse B220 PE-Cy7 (clone RA3-6B2; Biolegend; 1:200 dilution)

anti-mouse B220 BV785 (clone RA3-6B2; Biolegend; 1:200 dilution)

anti-mouse GL7 FITC (clone GL7; Biolegend; 1:100 dilution)

anti-mouse GL7 PerCP-Cy5.5 (clone GL7; Biolegend; 1:100 dilution)

anti-mouse CD3 PerCp-Cy5.5 (clone 17A2; Biolegend; 1:100 dilution)

anti-mouse CD38 PE (clone 90; Biolegend; 1:75 dilution)

anti-mouse CD38 PE-Cy7 (clone 90; Biolegend; 1:75 dilution)

anti-mouse CD38 BV711 (clone 90; Biolegened; 1:100 dilution)

anti-mouse CD83 PE (clone Michel-19; Biolegend; 1:100)

anti-mouse CD86 BV605 (clone GL-1; Biolegend; 1:400 dilution)

anti-mouse MHC II PE-Cy7 (clone M5/114.15.2; Biolegend; 1:400 dilution)

anti-mouse MHC II AF700 (clone M5/114.15.2; Biolegend; 1:400 dilution)

anti-mouse CD62L BV711 (clone MEL-14; Biolegend; 1:200 dilution)

anti-mouse CD62L PerCP-Cy5.5 (clone MEL-14; Biolegend; 1:200 dilution)

anti-mouse CD95 APC-R700 (clone SA367H8; Biolegend; 1:100 dilution)

anti-mouse CD138 BV711 (clone 281-2; Biolegend; 1:100 dilution) 
anti-mouse CD138 BV650 (clone 281-2; Biolegend; 1:100 dilution) anti-mouse IgD BV510 (11-26c.2a; Biolegend; 1:100 dilution)

anti-mouse IgD APC (11-26c.2a; Biolegend; 1:100 dilution)

anti-mouse CD4 APC-Cy7 (GK1.5; Biolegend; 1:100 dilution)

anti-mouse CD8-alpha APC-Cy7 (53-5.8; Biolegend; 1:100 dilution)

anti-mouse PD-L2 PE (TY25; Biolegend; 1:50 dilution)

anti-mouse PD-L2 BUV396 (TY25; BD; 1:50 dilution)

anti-mouse CD80 BV421 (16-10A; Biolegend; 1:50 dilution)

anti-mouse CD73 BV605 (TY/11.8; Biolegend; 1:50 dilution)

anti-mouse CD45.1 BV711 (A20; Biolegend; 1:100 dilution)

anti-mouse CD45.1 BV510 (A20; Biolegend; 1:50 dilution)

anti-mouse CD45.2 FITC (104; Biolegend; 1:50 dilution)

anti-mouse IgG1-biotin (RMG1-1; Biolegend; 1:50 dilution)

anti-mouse IgG1 PE/Dazzle 594 (RMG1-1; Biolegend; 1:50 dilution)

anti-mouse IgM APC (II/41; BD; 1:150 dilution)

biotinylated eOD-GT8 coupled to Streptavidin BV711 (Streptavidin purchased from Biolegend; 1:400 dilution)

anti-mouse Ly6C APC-Cy7 (clone HK1.4; Biolegend; 1:100 dilution)

anti-mouse Ly6G BV711 (clone 1A8; Biolegend; 1:100 dilution)

anti-mouse F4/80 PE (clone BM8; Biolegend; 1:50 dilution)

anti-mouse CD11c BV421 (clone N418; Biolegend; 1:100 dilution)

anti-mouse CD169 PE-Cy7 (clone 3D6.112; Biolegend; 1:50 dilution)

anti-mouse CD11b BUV395 (clone M1/70; BD; 1:100 dilution)

ELISAs:

Goat anti-mouse IgG-HRP (Bio-rad, 1:1000 dilution)

We relied on publications and validation cited by manufacturer. Links for each antibody are given below:

anti-mouse B220 (clone RA3-6B2, Biolegend)

https://www.biolegend.com/en-us/products/brilliant-violet-785-anti-mouse-human-cd45r-b220-antibody-7960

anti-mouse GL7 (clone GL7, Biolegend)

https://www.biolegend.com/en-us/products/fitc-anti-mouse-human-gl7-antigen-t-and-b-cell-activation-marker-antibody-8284

anti-mouse CD3 (clone 17A2, Biolegend)

https://www.biolegend.com/en-us/products/percp-cyanine5-5-anti-mouse-cd3-antibody-5596

anti-mouse CD38 (clone 90, Biolegend)

https://www.biolegend.com/en-us/products/pe-anti-mouse-cd38-antibody-183

anti-mouse CD83 (Michel-19; Biolegend)

https://www.biolegend.com/en-us/products/pe-anti-mouse-cd83-antibody-3580

anti-mouse CD86 (GL-1; Biolegend)

https://www.biolegend.com/en-us/products/brilliant-violet-605-anti-mouse-cd86-antibody-7798

anti-mouse MHC II (M5/114.15.2; Biolegend)

https://www.biolegend.com/en-us/products/alexa-fluor-700-anti-mouse-i-a-i-e-antibody-3413

anti-mouse CD62L (MEL-14; Biolegend)

https://www.biolegend.com/en-us/search-results/brilliant-violet-711-anti-mouse-cd62l-antibody-10317

anti-mouse CD95 (SA367H8; Biolegend)

https://www.biolegend.com/en-us/products/apc-anti-mouse-cd95-fas-antibody-13906

anti-mouse CD138 (281-2; Biolegend)

https://www.biolegend.com/en-us/search-results/brilliant-violet-650-anti-mouse-cd138-syndecan-1-antibody-8800

anti-mouse IgD (11-26c.2a; Biolegend)

https://www.biolegend.com/en-us/products/brilliant-violet-510-anti-mouse-igd-9032

anti-mouse CD4 (GK1.5; Biolegend)

https://www.biolegend.com/en-us/products/apccyanine7-anti-mouse-cd4-antibody-1964

anti-mouse CD8-alpha (53-5.8; Biolegend)

https://www.biolegend.com/en-us/products/apc-cyanine7-anti-mouse-cd8a-antibody-2269

anti-mouse PD-L2 (TY25; Biolegend)

https://www.biolegend.com/en-us/products/pe-anti-mouse-cd273-b7-dc--pd-I2-antibody-2547

anti-mouse CD80 (16-10A; Biolegend)

https://www.biolegend.com/en-us/products/pe-anti-mouse-cd80-antibody-43

anti-mouse CD73 (TY/11.8; Biolegend)

https://www.biolegend.com/nl-nl/products/brilliant-violet-605-anti-mouse-cd73-antibody-8153

anti-mouse CD45.1 (A20; Biolegend)

https://www.biolegend.com/en-us/products/brilliant-violet-711-anti-mouse-cd45-1-antibody-8925

anti-mouse CD45.2 (104; Biolegend)

https://www.biolegend.com/en-us/products/fitc-anti-mouse-cd45-2-antibody-6

anti-mouse IgG1 (RMG1-1; Biolegend)

https://www.biolegend.com/en-us/search-results/pe-dazzle-594-anti-mouse-igg1-antibody-14778

anti-mouse Ly6C (clone HK1.4, Biolegend)

https://www.biolegend.com/en-us/products/apccyanine7-anti-mouse-ly-6c-antibody-6758

anti-mouse Ly6G (clone 1A8, Biolegend)

https://www.biolegend.com/nl-nl/products/brilliant-violet-711-anti-mouse-ly-6g-antibody-12062

anti-mouse F4/80 (clone BM8, Biolegend)

https://www.biolegend.com/en-us/products/pe-anti-mouse-f4-80-antibody-4068

anti-mouse CD11c (N418, Biolegend)

https://www.biolegend.com/en-us/products/brilliant-violet-421-anti-mouse-cd11c-antibody-7149

anti-mouse CD169 (clone 3D6.112, Biolegend) 
https://www.biolegend.com/ja-jp/products/pe-cy7-anti-mouse-cd169-siglec-1-antibody-9929

anti-mouse CD35 (clone 8C12, BD)

https://www.bdbiosciences.com/eu/applications/research/b-cell-research/surface-markers/mouse/bv421-rat-anti-mouse-

cd35-8c12/p/740029

anti-mouse IgM (II/41; BD)

https://www.bdbiosciences.com/us/applications/research/b-cell-research/immunoglobulins/mouse/apc-rat-anti-mouse-igm-

ii $41 / p / 550676$

anti-mouse CD11b (clone M1/70; BD)

https://www.bdbiosciences.com/us/applications/research/stem-cell-research/mesenchymal-stem-cell-markers-bone-marrow/ mouse/negative-markers/buv395-rat-anti-cd11b-m170/p/563553

anti-mouse Goat IgG-HRP (H+L) (Bio-rad, Catalog 170-6516): validated by ELISA

biotinylated eOD-GT8 coupled to Streptavidin BV711 (Biolegend): validated by flow cytometry and previous publication (Abbott, et al., https://doi.org/10.1016/j.immuni.2017.11.023)

\section{Eukaryotic cell lines}

Policy information about cell lines

Cell line source(s)

Ramos B cells expressing germline VRC01 were obtained from Daniel Lingwood (Ragon Institute). FreeStyle 293-f was obtained from ThermoFisher. HEK293T were obtained from ATCC. The TZM-bl cell line engineered from CXCR4-positive HeLa cells to express CD4, CCR5, and a firefly luciferase reporter gene (under control of the HIV-1 LTR) was obtained from the NIH AIDS Research and Reference Reagent Program, Division of AIDS, NIAID, NIH (developed by Dr. John C. Kappes, and Dr. Xiaoyun $\mathrm{Wu}$ ).

Authentication

Ramos B cells expressing germline VRC01 were validated by flow cytometry. TZM-bl cell line from HeLa cells were validated by luciferase assay. FreeStyle 293-f and HEK293T were not validated.

Mycoplasma contamination

All cell lines tested negative for mycoplasma.

Commonly misidentified lines (See ICLAC register)

No commonly misidentified cell lines were used.

\section{Animals and other organisms}

Policy information about studies involving animals; ARRIVE guidelines recommended for reporting animal research

Laboratory animals

Balb/c, female, 8 week old mice were used for mouse immunizations. C57BL/6, male, 8 week old mice were used for the adoptive transfer experiments. New Zealand white rabbits, female, $2.5-3.0 \mathrm{~kg}$, 3-4 months old, were used for the rabbit immunizations.

Wild animals

No wild animals were used.

Field-collected samples

No field-collected samples were used.

Ethics oversight

Experiments and handling of mice were conducted under federal, state, and local guidelines under an IACUC approved protocol through MIT or La Jolla Institute for Immunology.

Note that full information on the approval of the study protocol must also be provided in the manuscript.

\section{Flow Cytometry}

\section{Plots}

Confirm that:

Х The axis labels state the marker and fluorochrome used (e.g. CD4-FITC).

Х The axis scales are clearly visible. Include numbers along axes only for bottom left plot of group (a 'group' is an analysis of identical markers).

$\triangle$ All plots are contour plots with outliers or pseudocolor plots.

$\triangle$ A numerical value for number of cells or percentage (with statistics) is provided.

\section{Methodology}

Sample preparation

Instrument

Software

Cell population abundance
Lymph nodes and spleens were mechanically digested, filtered into single cell suspensions, and stained using antibodies described above.

BD Canto and BD Fortessa were used for data collection. BD Aria was used for B cell sorting.

Flow cytometry data was analyzed using FlowJo.

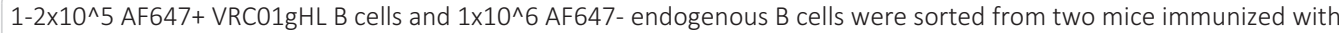
pSereOD-GT5/alum or pSer-eOD-GT8/alum for visualization of alum by TEM. For bulk RNA-seq analysis, $0.5 \times 10^{\wedge} 4-1 \times 10^{\wedge} 5$ 
VRC01gHL B cells were sorted from each mouse directly into TRIzol LS for further processing and therefore purity of post-sort fractions were not determined.

Gating strategy

Lymphocytes were gated on the starting cell population in a FCS/SSC plot, followed by FCA/FCH plot to gate single cells. Live cells were gated using either Aqua or Fixable Viability Dye e780. Cells were then gated on B220 positive cells. For adoptive transfer experiments, VRCO1 cells were identified using GFP expression and labeling with CTV.

$\bigotimes$ Tick this box to confirm that a figure exemplifying the gating strategy is provided in the Supplementary Information. 